
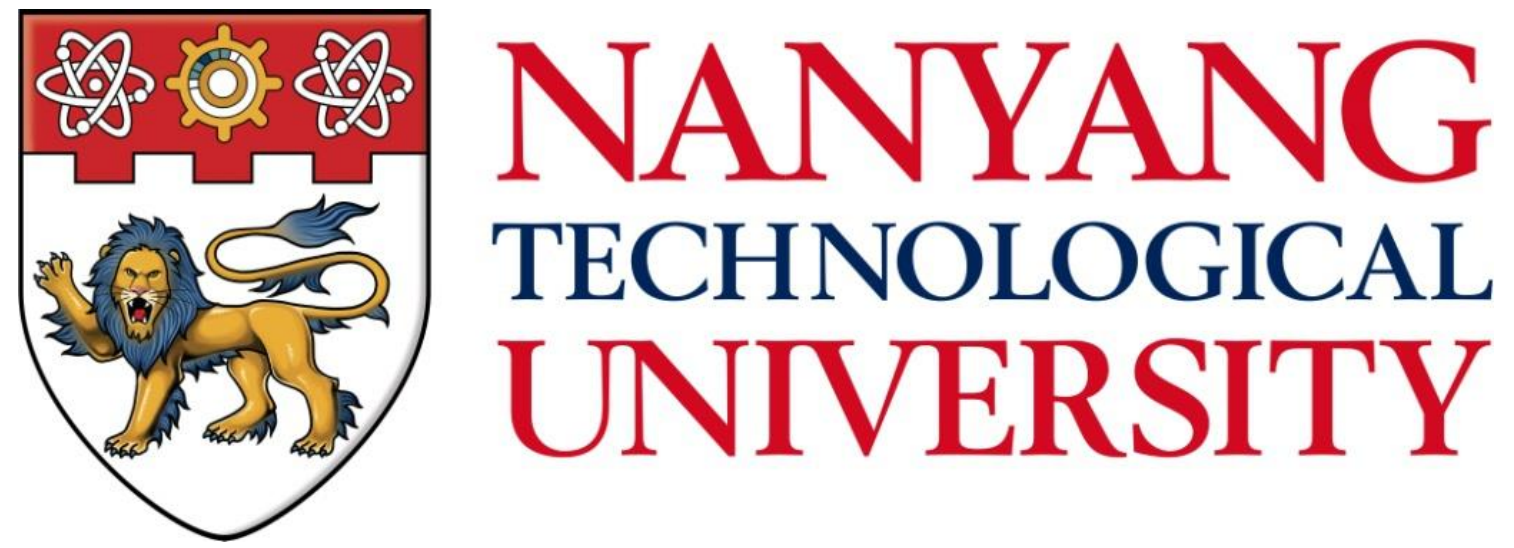

\title{
A STUDY of BIG DATA COMPUTING PLATFORMS: PERFORMANCE, FAIRNESS AND ENERGY CONSUMPTION
}

NIU ZHAOJIE

Interdisciplinary Graduate School IGS-LILY 2017 


\section{A STUDY of BIG DATA COMPUTING PLATFORMS: PERFORMANCE, FAIRNESS AND ENERGY CONSUMPTION}

NIU ZHAOJIE

\section{Interdisciplinary Graduate School IGS-LILY}

A thesis submitted to Nanyang Technological University in fulfillment of the requirement for the degree of

Doctor of Philosophy 


\section{Abstract}

Today, population aging is a trend spreading across the world. It is estimated that the number of people aged 60 years or over, is increasing rapidly. Although the elderly has wisdom and wealth gathered from their life experience, they often require long-term assistance from others. Ambient assisted living (AAL) systems open up a new opportunity to address the needs of aged by utilizing information and communication techniques. Multiple sensors and actuators coexist in ambient assisted living systems and are deployed everywhere surrounding elderly. A huge volume of sensed data is collected continuously from elderly, storage of these massive raw data and efficient processing on them to infer knowledge from the data and also guide the actuators to meet the needs of elderly is a problem we face. In the thesis, we mainly focus on the efficient large-scale data processing, we investigate whether and how we can build an effective large-scale data processing system to effectively excavate knowledge, wisdom and skills from elderly people to impact the development of the entire society.

A lot of large-scale big data analytics systems have thereby emerged to process the massive data effectively. Efficient job scheduling and resource management for these data analytic frameworks are nontrivial. Modern job schedulers and resource coordinators in data processing frameworks often need to consider multiple objectives simultaneously due to various system operators requirements on data analytics. Currently, resource efficiency (throughput), job latency (per-job performance), fairness (isolation guarantee) and energy consumption are important concerns for the job scheduler in modern large-scale multiple-tenant environments. Resource efficiency is de facto the very important factor for the big data analytic framework. Job latency reflects the waiting time of the application. Fairness is a key building block of any multiple-tenant computing system that allows resource sharing effectively. Energy usage of the data center has reached $3 \%$ of the global electricity consumption while generating 200 million metric tons of CO2 in 2014. In order to reduce carbon emission and financial burden on the electricity, a lot of data centers have been re-designed and powered with multiple energy sources, including renewable (green) energy from non-polluting sources and brown energy from traditional polluting sources.

Improving the resource efficiency and reducing the per-job latency are the common sense on those large-scale data processing frameworks and fruitful studies are proposed in these directions. In 
contrast, the fairness and energy consumption of those frameworks need further exploration and how the resource efficiency, job latency, fairness and energy consumption interact each other on big data computing frameworks is not well addressed. In the thesis, we perform detailed study on the resource efficiency, job latency, fairness and energy consumption of big data processing frameworks and find that these objectives can be translated to discordant actions. We propose bi-criteria optimization algorithms and finally implement a general multiple-objective optimization system to address the tradeoff between different objectives.

First, we explore the tradeoff between resource efficiency and fairness in detail and find that the tradeoff is related to the workload. Therefore, we should develop a scheduler to be aware of workload dynamics and the efficiency-fairness tradeoff. Since researchers keep inventing new scheduling algorithms, we develop a meta-scheduler called FLEX which addresses the efficiency-fairness tradeoff by leveraging existing efficiency- and/or fairness-optimized schedulers. FLEX adaptively chooses the most suitable scheduler at runtime according to the variation of the workload and user-defined SLAs. We have implemented FLEX in Hadoop YARN. We conduct experiments with real deployment in a local cluster and perform simulation studies with production traces. Flex performs better than the state-of-the-art scheduling algorithm in two aspect: 1) Given a predefined threshold on the fairness loss, FLEX reduces the makespan by up to $22 \%$ and $24 \%$ in real deployment and large-scale simulation, respectively; 2) Given the predefined threshold on the makespan reduction, it reduces the fairness loss by up to $75 \%$ and $73 \%$ in real deployment and large-scale simulation, respectively.

Second, we study the tradeoff between resource efficiency and energy consumption in the modern datacenters which have been re-designed and integrated with the intelligence of smartly drawing power from multiple sources, including green energy from renewable and non-polluting sources and brown energy is drawn from electric grid when renewable is insufficient. We find that not all joules are equal in sense that the amount of work that can be done by a joule can vary significantly. We investigate how to exploit such joule efficiency to maximize the benefits of renewable energy and dynamic pricing for MapReduce framework while satisfying the workload deadline. We have developed JouleMR, a cost-effective and green-aware MapReduce framework. We implement JouleMR on top of hadoop YARN, and evaluate it in both real local cluster and large-scale simulator. JouleMR significantly reduces the brown energy on both real experiments and simulations (up to $35 \%$ and $28 \%$ reduction compared with the state-of-the-art systems). Additionally, Joule MR reduces the electricity cost on both real experiments and simulations compared to the state-of-the-art work (by $30 \%$ and $36 \%$ reduction, respectively).

Third, we further explore the relationship between multiple objectives and develop an efficient framework for multi-objective optimizations on geo-distributed data analytics systems. We observe 
that these objectives can be translated to discordant actions and their relationship can be impacted by the unique features of geo-distributed data analytics systems. We formulate the multi-objective optimization problem mathematically and propose an efficient online heuristic algorithm to perform the multi-objective optimization for the geo-distributed data analytics systems. We develop GeoSpark, an extension to Spark, which automatically performs a multi-objective optimization according to the system operators preferences on different objectives. GeoSpark effectively performs the multiobjective optimizations based on system operators preferences on different objectives. GeoSpark achieves up to $30 \%$ makespan reduction, $28 \%$ job latency reduction and better fairness guarantee compared with existing schedulers in Apache Spark in the geo-distributed setting.

In summary, this thesis mainly aims at the resource efficiency, job latency, fairness and energy consumption of the job scheduler and their relationships for large-scale data computing frameworks . We propose bi-criteria optimization algorithms to address the tradeoff between different objectives and finally develop a general multiple-objective optimization system. 


\section{Acknowledgments}

Firstly, I express my thanks to my previous supervisor Dr. Bingsheng He, my current supervisor Dr. Lau Chiew Tong, my co-supervisor Dr. Ming Jian and my mentor Dr. Zhenchang Xing for their continuous, remarkable and patient guidance throughout my four year's study. Their immense knowledge, invaluable advice and unstinting encouragement have helped me a lot in my research.

I would like to thank my friends and colleagues in IGS-LILY and PDSL. I will always remember all the great memories we created together in the part four years.

I gratefully acknowledge the scholarship provided by LILY, IGS(Interdisciplinary Graduate School) and Nanyang Technological University for my doctorial program.

Last but not least, I want to thank my family and friends for their unwavering support throughput my $\mathrm{PhD}$. 


\section{Contents}

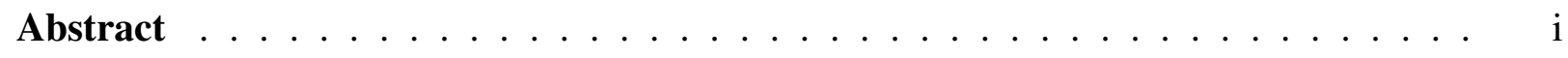

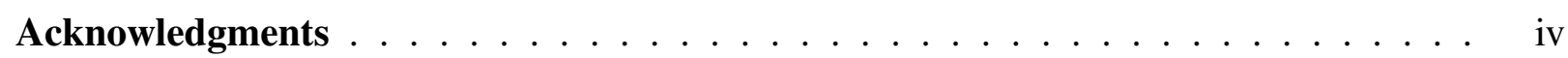

List of Figures $\ldots \ldots \ldots \ldots \ldots \ldots \ldots \ldots \ldots \ldots \ldots \ldots \ldots \ldots \ldots \ldots \ldots$

1 Introduction 1

1.1 Background of Big Data Processing $\ldots \ldots \ldots \ldots \ldots \ldots \ldots$

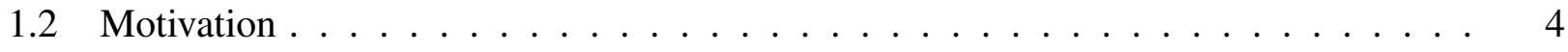

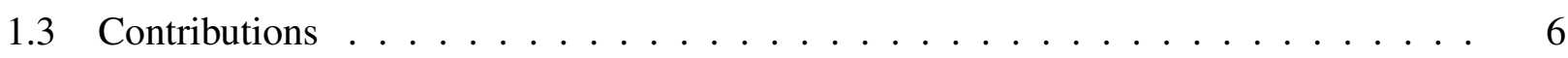

1.4 Structure of the Thesis $\ldots \ldots \ldots \ldots \ldots \ldots \ldots$

2 Background and Related Work 9

2.1 Hadoop YARN . . . . . . . . . . . . . . . . . . . . . . 9

2.2 Efficiency-oriented Scheduling . . . . . . . . . . . . . . . . 11

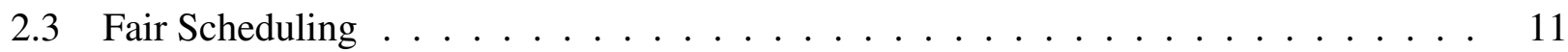

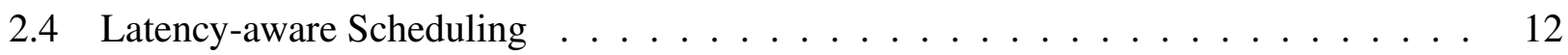

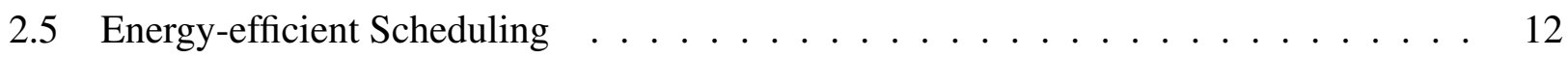

2.6 Cost-effective Scheduling . . . . . . . . . . . . . . . . . . . . . 13

2.7 Green-aware Scheduling . . . . . . . . . . . . . . . . . . . . . . 14

2.8 Multi-objective Optimizations in Job Scheduling $\ldots \ldots \ldots \ldots \ldots$

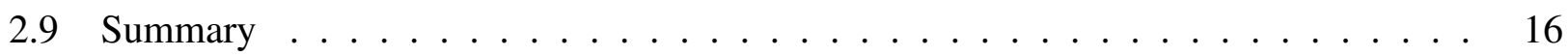

3 An Adaptive Efficiency-Fairness Meta-scheduler for Data-Intensive Computing 17

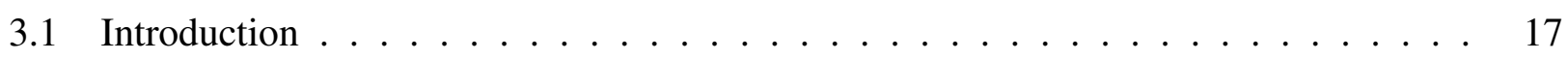

3.2 Workload Characterization Model and Problem Statement _ . . . . . . . . 20

3.2.1 Workload Characterization Model . . . . . . . . . . . . . . . 21

3.2 .2 Problem Statement . . . . . . . . . . . . . . . . . . . . 23 
3.3 System Design of Meta-scheduler . . . . . . . . . . . . . . . . 25

3.3.1 Rationale of System Design . . . . . . . . . . . . . . . 25

3.3 .2 System Overview . . . . . . . . . . . . . . . . . . . . . . 26

3.3.3 Detailed Design of FLEX . . . . . . . . . . . . . . 27

3.3.4 Implementation on Hadoop YARN . . . . . . . . . . . . . . . 31

3.4 Evaluation . . . . . . . . . . . . . . . . . . . . 33

$3.4 .1 \quad$ Experiment Setup . . . . . . . . . . . . . . . . . . . 33

3.4.2 Real Deployment Evaluations . . . . . . . . . . . . . . 35

3.4.2.1 Overall Comparison . . . . . . . . . . . . . 36

3.4.2.2 Runtime Overhead Analysis . . . . . . . . . . . . . . . . . . 40

3.4.2.3 Model Evaluation . . . . . . . . . . . . . . 42

3.4.2.4 Impact of Long-term Fairness Definitions . . . . . . . . . . . . 45

3.4 .3 Trace-driven Simulations . . . . . . . . . . . . . . . . . . . 47

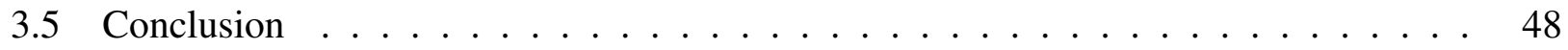

4 JouleMR: Towards Cost-Effective and Green-Aware Data Processing Frameworks 49

4.1 Introduction . . . . . . . . . . . . . . . . . . . . . . . . . 49

4.2 Problem Definition and Overall Design _ . . . . . . . . . . . . . . . . 52

4.2 .1 Problem Definition . . . . . . . . . . . . . . . . . . 52

4.2 .2 System Overview . . . . . . . . . . . . . . . . . . . . 58

4.3 Green-aware Scheduling . . . . . . . . . . . . . . . . . 61

4.3.1 Basic Energy-efficient Plan . . . . . . . . . . . . . . . . . . . 62

4.3.2 Green-aware Optimizations with Job Transformations . . . . . . . . . . 64

4.3.3 Battery Assisted Green Shifting . . . . . . . . . . . . . . . . . 67

4.3 .4 Cost Model . . . . . . . . . . . . . . . . . . . . . . . . . . . . . 69

4.3.5 Server Power Management . . . . . . . . . . . . . . . . . . . . 73

4.4 Cost-effective Scheduling . . . . . . . . . . . . . . . . . . . . . 74

4.4.1 Basic Energy-efficient Plan . . . . . . . . . . . . . . . . . . 74

4.4.2 Cost-effective Optimizations with Job Transformations . . . . . . . . . . . 76

4.4.3 Battery Assisted Cheap Brown Shifting . . . . . . . . . . . . . . . 76

4.5 Evaluation . . . . . . . . . . . . . . . . . . . . 77

4.5.1 Experiment Setup . . . . . . . . . . . . . . . . . . . 77

4.5.1.1 Micro Benchmark Setup . . . . . . . . . . . . . . . 79 
4.5.1.2 Simulation Setup _. . . . . . . . . . . . . 80

4.5.2 Green-aware Scheduling: Micro Benchmark Study . . . . . . . . . . . . 81

4.5.2.1 Model Accuracy . . . . . . . . . . . . . . . . . . . 81

4.5.2.2 Overall Comparison . . . . . . . . . . . . . . . 82

4.5.3 Green-aware Scheduling: Large-scale Trace Study . . . . . . . . . . . . . 84

4.5.3.1 Simulation Validation . . . . . . . . . . . . . . . 84

4.5.3.2 Overall Comparison . . . . . . . . . . . . . . . . 85

4.5 .4 Sensitivity Studies . . . . . . . . . . . . . . . . . 86

4.5.5 Results on Cost-effective Scheduling . . . . . . . . . . . . . . 91

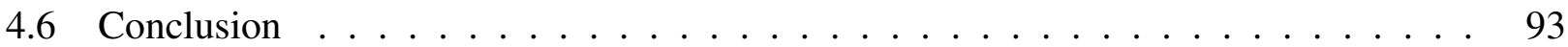

5 Multi-objective Optimizations in Geo-distributed Data Analytics Systems 94

5.1 Introduction . . . . . . . . . . . . . . . . . . . . . . . . . 94

5.2 Background and Motivation _ . . . . . . . . . . . . . . . . 97

5.2.1 Job Scheduling in Geo-distributed DCs . . . . . . . . . . . . . . . . . . 98

5.2.2 Motivating Examples . . . . . . . . . . . . . . . . . . 100

5.2.2.1 Intermediate Data Distribution for trade-off (resource efficiency vs.

fairness $\ldots \ldots \ldots \ldots \ldots \ldots$

5.2.2.2 WAN Link Bandwidths for trade-off (resource efficiency vs. fairness) 102

5.2.2.3 Summary. . . . . . . . . . . . . . . 103

5.3 Multi-objective Optimization . . . . . . . . . . . . . . . . . . 103

5.3.1 Problem Formulation . . . . . . . . . . . . . . . . . . . 103

5.3.2 Light-Weight Scheduling Algorithm . . . . . . . . . . . . . . . 106

5.3.3 Visualizing Pareto Fronts and Calibrating Weights . . . . . . . . . . . 110

5.4 Evaluation . . . . . . . . . . . . . . . . . . . . . 111

$5.4 .1 \quad$ Experimental Setup . . . . . . . . . . . . . . . . . . 111

5.4 .2 Overall Results . . . . . . . . . . . . . . . . . . . . 112

5.4 .3 Sensitivity Study . . . . . . . . . . . . . . . . . . . . . 114

5.4 .4 Overhead Analysis . . . . . . . . . . . . . . . . . . 118

5.4 .5 Summary . . . . . . . . . . . . . . . . . . . 118

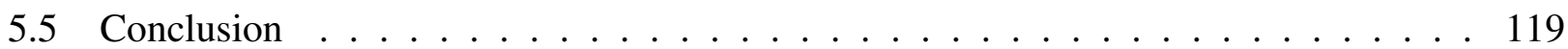


6 Conclusion and Future Work $\quad 120$

6.1 Conclusion . . . . . . . . . . . . . . . . . . 120

6.2 Future Work . . . . . . . . . . . . . . . . . . . . . . 120

6.2.1 Big Data Processing on Biomedical and Health-care Fields . . . . . . . . . 121

6.2.2 Orchestration Scheduler for Containers . . . . . . . . . . . . . . . . 122

6.2.3 Heterogeneous Data Processing with Actor Model . . . . . . . . . . . . . . 122

Bibliography . . . . . . . . . . . . . . . . . . . . . . . 124 


\section{List of Figures}

1.1 The reference architecture of a cloud based Ambient Assisted Living system in LILY [1]. This AAL system consists of three parts: data sensing; data storage and processing; control and feedback . . . . . . . . . . . . . . . . . . 3

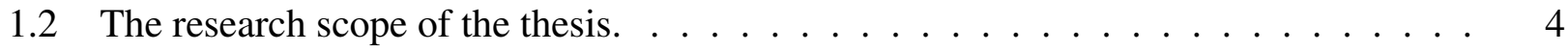

2.1 The overview of Hadoop YARN $\ldots \ldots \ldots \ldots$

3.1 Heterogeneous resource demand for tasks from Google traces [2] . . . . . . . . . . 19

3.2 Tradeoff between resource efficiency and fairness for workloads with different complementary degrees . . . . . . . . . . . . . . . . . . . . 20

3.3 The workloads with different resource demands. . . . . . . . . . . . . . 21

3.4 Design of the meta-scheduler FLEX . . . . . . . . . . . . . . . . . . 27

3.5 An example decision tree trained with the default parameter setting . . . . . . . . . 30

3.6 FLEX implementation on Hadoop YARN. The modified existing components are shown with green rectangles and the newly added components are shown with blue rectangles. Their corresponding event dispatchers are shown with grey round rectangles. The newly added modules of the component are shown with blue round rectangles. 33

3.7 The reduction on the makespan and fairness loss of FLEX compared to Tetris (Facebook workload $\ldots \ldots \ldots \ldots$. . . . . . . . . . . . . . 37

3.8 The reduction on the makespan and fairness loss of FLEX compared to Tetris (Google workload $\ldots \ldots \ldots \ldots \ldots \ldots \ldots$

3.9 Resource utilization of the cluster under FLEX and Tetris during the execution . . . . 38

3.10 The scheduler selection ratio . . . . . . . . . . . . . . . . . . . 39

3.11 One scheduler $(4$ combinations in total $) \ldots \ldots \ldots$. . . . . . . . . . 40

3.12 Two schedulers $(6$ combinations in total $) \ldots \ldots \ldots \ldots$. . . . . . . . . . 41

3.13 Three schedulers $(4$ combinations in total $) \ldots \ldots \ldots \ldots$ 
3.14 Confusion matrix $($ Facebook workload $) \ldots \ldots \ldots \ldots$

3.15 Classification accuracy of the decision tree (Facebook workload) . . . . . . . . . 43

3.16 Confusion matrix (Different workloads) . . . . . . . . . . . . . . . 44

3.17 Classification accuracy of the decision tree (Different workloads) . . . . . . . . . . 44

3.18 The reduction on the makespan and fairness loss of FLEX compared with Tetris for different prediction errors . . . . . . . . . . . . . . . . 45

3.19 Tradeoff between the efficiency and fairness of efficiency-oriented scheduler for workloads with different complementary degrees. (long-term fairness) . . . . . . . . . . .

3.20 The reduction on the makespan and fairness loss of FLEX compared to Tetris (longterm fairness $) \ldots \ldots \ldots \ldots \ldots \ldots \ldots$

3.21 The reduction on the makespan and fairness loss of FLEX compared to Tetris in largescale simulation with Google trace . . . . . . . . . . . . . . .

4.1 Energy consumption and performance tradeoff of Hadoop YARN in our local cluster. " $x \mathrm{~N}$ " means the result is obtained by running on $x$ nodes. Selectivity is defined as the ratio between the amount of output data of the map phase and the total input size of the benchmark (500GB in the compressed format). . . . . . . . . . . . 52

4.2 System overview of JouleMR . . . . . . . . . . . . . . . . . 53

4.3 Two illustrative examples: (Top) SDE reduces the brown energy consumption; (bottom) SDE increases the brown energy consumption. . . . . . . . . . . . . . 59

4.4 An example of battery assisted green shifting . . . . . . . . . . . . . 68

4.5 The price of brown energy over time . . . . . . . . . . . . . . 75

4.6 Two illustrative examples: (a) Delay and promote operation reduces the electricity cost; (b) Delay and promote operation increases the electricity cost. . . . . . . . . . .

4.7 A snapshot of solar trace gained from MIDC, and a mixed workload simulating Google's search jobs . . . . . . . . . . . . . . . . . . 80

4.8 Estimated and measured energy consumption of individual jobs . . . . . . . . . . 82

4.9 Overall comparison of green-aware optimizations with the micro benchmark . . . . 83

4.10 Validation of the green-aware simulations on replayed Facebook trace . . . . . . . 84

4.11 Overall comparison of green-aware optimizations with the Facebook production workloads . . . . . . . . . . . . . . . . . . 86

4.12 Detailed energy consumption of different green-aware optimizations $\ldots$. . . . . . 86

4.13 The brown energy usage varying the battery capacity $\ldots \ldots \ldots$. . . . . . . . 87

4.14 The brown energy usage varying slacks . . . . . . . . . . . . . 88 
4.15 The brown energy usage varying workload prediction errors $\ldots \ldots$. . . . . . . 88

4.16 The brown energy usage varying the scale of solar energy . . . . . . . . . . . . 89

4.17 The brown energy usage varying the epoch size . . . . . . . . . . . . . . 89

4.18 Brown energy usage when solar trace is highly variable . . . . . . . . . . . . . 90

4.19 The brown energy usage varying the cluster size . . . . . . . . . . . . . . . 90

4.20 Overall comparison of cost-effective optimizations with the micro benchmark . . . . 91

4.21 Overall comparison of cost-effective optimizations with the Facebook production workloads . . . . . . . . . . . . . . . . . . . . . . 92

4.22 Validation of the cost-effective simulations on replayed Facebook trace . . . . . . . . 92

4.23 Detailed brown usage of different cost-effective optimizations . . . . . . . . . . 93

5.1 System architecture of geo-distributed job scheduling . . . . . . . . . . . . . 98

5.2 The amount of cross-DC data transfer for different placement of one reduce task. . . 101

5.3 Illustration example of the trade-offs between resource efficiency and fairness impacted by the intermediate data distribution (Two free slots in $D 1$, efficiency-aware scheduler assigns 2 tasks of job 1 to $D 1$ and fair scheduler assigns 1 task of job 1 and 1 task of job 2 to D1.) . . . . . . . . . . . . . . . . . . . . . 101

5.4 Illustration example of the trade-offs between resource efficiency and fairness impacted by the heterogeneous WAN link bandwidth ( $D 1$ and $D 2$ each has 2 available slots. Efficiency-aware scheduler assigns 2 tasks of job 1 to $D 1$ and 2 tasks of job 2 to $D 2$. Fair scheduler assigns 1 task of job 1 to $D 1,1$ task of job 2 to $D 1$ and 2 tasks of job 2 to D2.) . . . . . . . . . . . . . . . . . . . . . . . 102

5.5 Bi-partitioned graph matching. . . . . . . . . . . . . . . . 107

5.6 Resource efficiency comparison of different schedulers $\left(W_{e}=1, W_{q}=0, W_{f}=0\right.$ for GeoSpark). . . . . . . . . . . . . . . . . . . 112

5.7 The amount of data to transfer across different DCs for different schedulers. . . . . 113

5.8 Average resource utilizations on CPU and memory for different schedulers. . . . . . 113

5.9 Job latency comparison of different schedulers $\left(W_{e}=0, W_{q}=1, W_{f}=0\right.$ for GeoSpark). 114

5.10 Completion time of two jobs under different schedulers $\left(W_{e}=0, W_{q}=0, W_{f}=1\right.$ for GeoSpark). . . . . . . . . . . . . . . . . 114

5.11 Fairness loss of different schedulers (baseline: GeoSpark $\left.\left(W_{e}=0, W_{q}=0, W_{f}=1\right)\right)$. 115

5.12 Makespan of GeoSpark by varying the weight on resource efficiency. . . . . . . . . 115

5.13 Trade-off between resource efficiency and fairness. . . . . . . . . . . . . . 116

5.14 Impact of data skewness on different optimization objectives. . . . . . . . . . . . 116 
5.15 Impact of network heterogeneity on different optimization objectives. . . . . . . . 117

5.16 Impact of the number of DCs on different optimization objectives. . . . . . . . . 118 


\section{Chapter 1}

\section{Introduction}

This chapter first introduces the background of big data processing systems. Then we present the major motivations which are involved in the tradeoffs between resource efficiency, job latency, fairness and energy consumption of the big data processing frameworks. Finally, we present the contributions, followed by the organization of the thesis.

\subsection{Background of Big Data Processing}

Today, population aging is a trend spreading across the world. It is estimated that one third of the population in developed countries and one fifth of that in developing countries will be elderly people by the year of 2050 [3]. In Singapore, the proportion of residents age 65 years or above rose from $9.3 \%$ in 2011 to $9.9 \%$ in 2012 [4]. On the one hand, a large aging population imposes significant pressure to the medical and healthcare systems. Government and business have set up initiatives to address various issues risen from global aging. On the other hand, there are vast opportunities in enabling elderly citizens to continue making important contributions to society, which will derive enormous benefits. The posses invaluable knowledge, wisdom and skills accumulated over many years. With the economic and social development, more and more elderly people have high education background, according to statistic up to 2010 [5, 4], there are 3.7 percent of Singapore residents age 65 years or above have received university education. In Active Living for the Elderly (LILY) center, 
we will investigate weather and how we can leverage knowledge, wisdom and skills from elderly people to impact the development of the entire society.

Building knowledge base for elderly people, especially for those high education background people, will reap huge economic and social benefits. Firstly, knowledge and experience from elderly people is highly dependable and precious for next generation people, and young people can learn more which may not be always available from books. Secondly, even though aging people cannot finish high demand labor works, they still can continue making important contributions to society taking advantage of computing technologies. There are many opportunities to leverage their knowledge and experience in different sectors [4]. Thirdly, in modern societies, continuing their contributions to the society attract more and more elderly people. They can find their personal fulfillment by continuously contributing their knowledge to the society. Moreover, the knowledge base itself forms a social network among generations and will mitigate the feeling of loneliness.

Although the elderly have wisdom and wealth gathered from their life experience, they other require long-term assistance. Ambient assisted living (AAL) system open up a new opportunity to address the needs of aged by utilizing information and communication techniques [6]. Figure 1.1 shows a typical reference architecture of cloud-based AAL systems. Multiple sensors and actuators coexist in ambient assisted living systems and are deployed everywhere surrounding elderly. A huge volume of sensed data is collected continuously from elderly, storage of these massive raw data and efficient processing on them to infer knowledge from the data and also guide the actuators to meet the needs of elderly is a problem we face. Essentially, we envision that building a knowledge base for elderly is a big data problem which needs the support of powerful data processing platform in large cluster. In the thesis, we mainly target the efficient data processing part and we investigate whether and how we can build an effective large-scale data processing systems to effectively excavate knowledge, wisdom and skills from elderly people to impact the development of the entire society.

A lot of large-scale big data analytics platforms have thereby emerged and become more and more popular recently $[7,8,9,10,11]$ in the current era of "big data". Resource efficiency, job latency, 


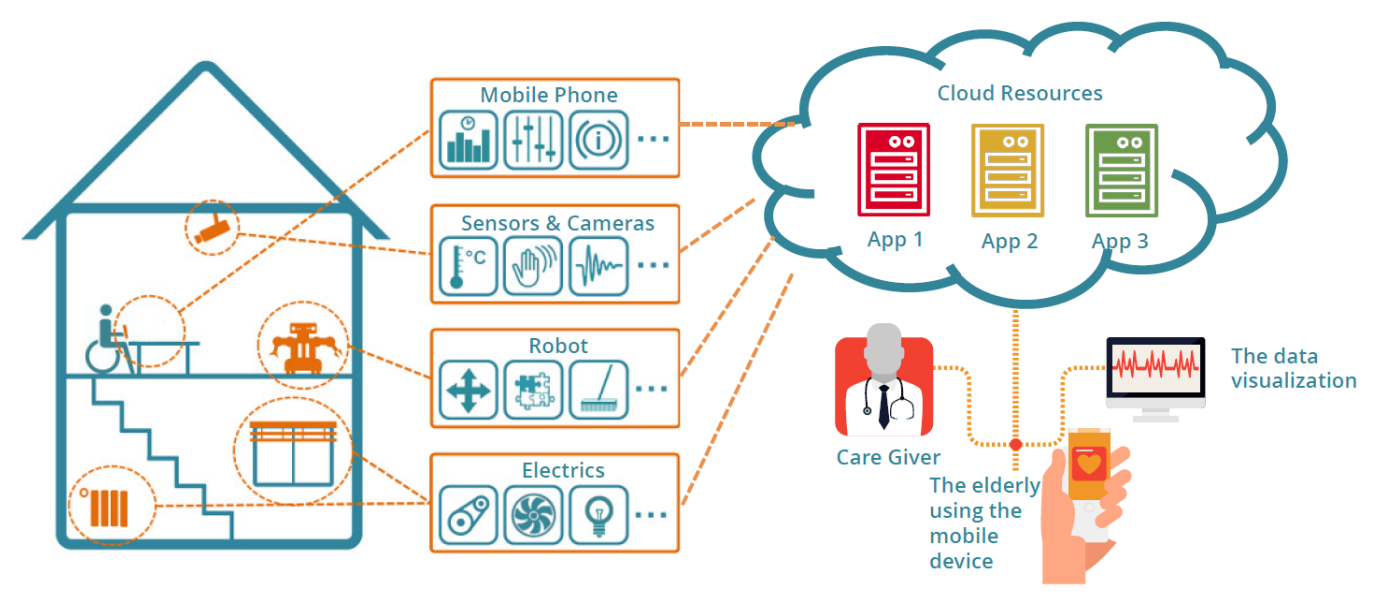

Figure 1.1: The reference architecture of a cloud based Ambient Assisted Living system in LILY [1]. This AAL system consists of three parts: data sensing; data storage and processing; control and feedback

fairness and energy consumption are important concerns for cluster providers and users in the largescale multi-tenant environments.

- Resource efficiency is de facto the very important concern for the big data platforms in the large-scale cluster [11].

- Job latency reflects the job waiting time and it is a critical concern from the user aspect [12].

- Fairness is a key building block of any shared data processing platform which allows resource sharing more effectively [13].

- Energy usage of the data center has reached $3 \%$ of the global electricity consumption while generating 200 million metric tons of CO2 in 2014 [14]. In order to reduce carbon emission and financial burden on the electricity, a lot of data centers have been re-designed and powered with multiple energy sources, including renewable (green) energy comes from non-polluting sources and brown energy from traditional polluting sources [15]. 


\subsection{Motivation}

Improving the resource efficiency and reducing job latency are the common sense on those largescale data processing frameworks and fruitful studies are proposed in these directions. In contrast, the fairness and energy consumption of those frameworks need further exploration and how the resource efficiency, job latency, fairness and energy consumption interact each other on big data computing frameworks is not well addressed. In our thesis, we perform detailed study on the resource efficiency, job latency, fairness and the energy consumption of those big data computing systems as well as their relationships. We find that these objectives can be translated to discordant actions and there are tradeoffs between them. We further explore what factors and how they impact resource efficiency, job latency, fairness and energy consumption of the big data processing frameworks. Based on the observations in our study, we propose bi-criteria optimization algorithms to address the tradeoff between different objectives and finally develop a general multiple-objective optimization system, as shown in Figure 1.2. Particularly, in this thesis proposal, we propose to explore the following research problems.

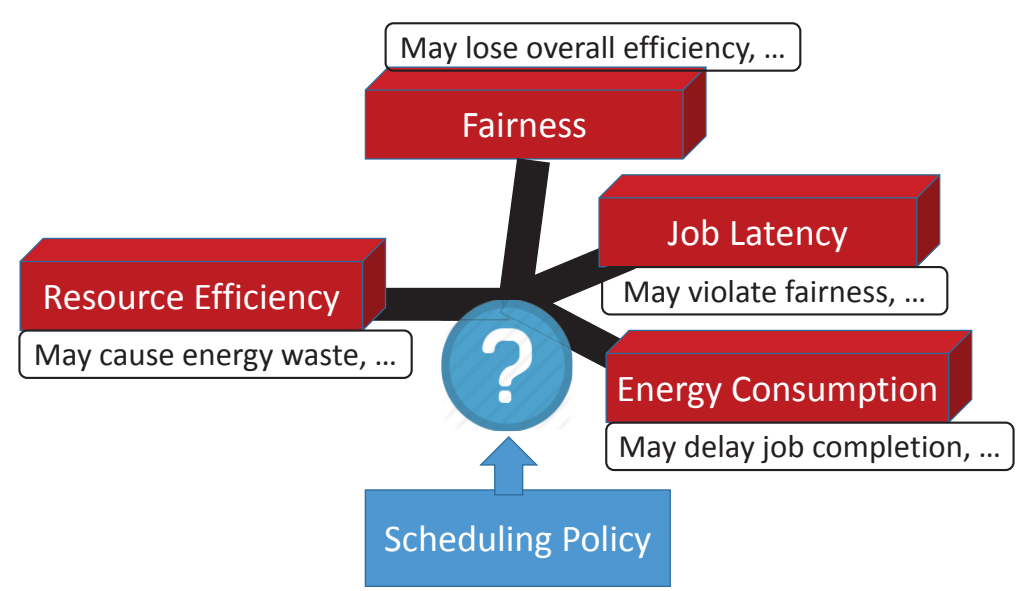

Figure 1.2: The research scope of the thesis.

Resource Efficiency vs. Fairness. First, as shown in Figure 1.2, we have observed that there is a tradeoff between resource efficiency and fairness because of resource contention between users 
and jobs. Recent studies proposed some bi-criteria optimization algorithms. However, these heuristic algorithms fail to address the tradeoff between resource efficiency and fairness. The reason is that, 1) the resource efficiency and fairness under different schedulers vary a lot due to their distinct optimization purposes; 2) due to the heterogeneous resource demands of the workload, a static scheduler cannot always achieve the most proper tradeoff between the resource efficiency and fairness with the variation of the resource demands of running workload during the computation.

Resource Efficiency vs. Energy consumption. Second, as shown in Figure 1.2, there is a tradeoff between resource efficiency and energy consumption in modern data centers which have been re-designed and integrated with the intelligence of smartly drawing power from multiple sources, including green energy from renewable and non-polluting sources and brown energy is drawn from electric grid when renewable is insufficient. It is because that the supply of the green energy cannot match the demand of the workload well as the renewable sources are intermittent due to the daily/seasonal effects. Therefore, some workload has to be delayed when the green energy is not available to match the renewable supply. Many green-aware algorithms and systems are proposed. However, these heuristics simply delay the workload according to the deadlines of the workload and little attention was paid to the efficiency of each joule consumed by the data analytics workloads. In fact, not all joules are equal in the sense that the amount of workload that can be done by a joule can vary significantly in data centers. Ignoring this facto leads to significant energy waste.

Considering all of them simultaneously. Third, as shown in Figure 1.2, modern job schedulers and resource coordinators in data processing frameworks often need to consider multiple objectives simultaneously due to various system operators requirements on data analytics. More importantly, these objectives can be translated to discordant actions [11] and thus make the multi-objective optimization quite challenging. For example, a fair scheduler such as DRF [12] may decide to give resources to a certain job while the efficiency-aware heuristic that maximizes resource efficiency may prefer a different job with better data locality. It is nontrivial to efficiently perform multiple-objective 
optimizations for the job schedulers in datacenters and achieve good trade-off between different objectives.

\subsection{Contributions}

In this thesis, we have explored three projects for workload-aware optimization, cost-effective and green-aware optimization, and multiple-objective optimization to address the tradeoffs between resource efficiency, job latency, fairness and energy consumption of big-data processing frameworks. The contributions of this thesis are summarized as follows:

Workload-aware Meta Scheduler. In order to address the tradeoff between resource efficiency and fairness, we first develop a workload characteristic model with the regression approach to estimate the resource efficiency improvement and the fairness loss given the workload. Next, we leverage existing efficiency- and/or fairness-optimized schedulers and propose a workload-aware meta scheduler called FLEX (means flexible scheduler) that adaptively chooses the most proper scheduler according to the current running workload and the user-defined Service Level Agreement (SLA). FLEX performs the bi-criteria optimization for resource efficiency and fairness. Given a predefined threshold on fairness loss, FLEX is able to maximize the resource efficiency of the cluster, or vice versa. We have implemented FLEX in Hadoop YARN [9]. We conduct experiments with real deployment in a local cluster and perform simulation studies with production traces. Flex performs better that the state-of-the-art scheduling algorithm in two aspect: 1) Given a predefined threshold on the fairness loss, FLEX reduces the makespan by up to $22 \%$ and $24 \%$ in real deployment and large-scale simulation, respectively; 2) Given the predefined threshold on the makespan reduction, it reduces the fairness loss by up to $75 \%$ and $73 \%$ in real deployment and large-scale simulation, respectively.

Energy-efficient and Green-aware Scheduler. In order to address the tradeoff between resource efficiency and energy consumption. We propose JouleMR, a cost-effective and green-aware MapReduce framework which is aware of joule efficiency to maximize the effectiveness of utilizing green 
energy as well as dynamic pricing. We develop job/task scheduling algorithms with special considerations on the factors on joule efficiency in the data center, including the energy efficiency of MapReduce workloads, renewable energy supply, dynamic pricing and the battery usage. We further develop a performance-energy consumption model which guides us to make the scheduling decision so that the deadline can be met and joule efficiency can be accurately estimated for scheduling the job/task at appropriate time. We implement JouleMR on top of hadoop YARN, and evaluate it in both real local cluster and large-scale simulator. JouleMR significantly reduces the brown energy on both real experiments and simulations (up to $35 \%$ and $28 \%$ reduction compared with the state-of-the-art systems). Additionally, JouleMR reduces the electricity cost on both real experiments and simulations compared to the state-of-the-art systems (by 30\% and 36\% reduction, respectively).

Multi-objective Optimization Framework. We further explore the multiple objectives simultaneously and eventually we develop a general framework which performs multi-objective optimizations for resource efficiency, job latency and fairness on geo-distributed data analytics systems. Specifically, we develop GeoSpark, an extension to Spark, which automatically performs a multi-objective optimization according to the system operators preferences on different objectives. The multi-objective optimization is inherently intractable especially for large-scale workloads. Therefore, we propose an efficient online heuristic to approximate the optimal scheduling plan while achieving a lower bound guarantee in the worst case. Evaluation using synthetic workload shows that GeoSpark effectively performs the multi-objective optimizations based on system operators preferences on different objectives. GeoSpark achieves up to $30 \%$ makespan reduction, $28 \%$ job latency reduction and better fairness guarantee compared with existing schedulers in Apache Spark in the geo-distributed setting.

\subsection{Structure of the Thesis}

The report is organized into five chapters.

Chapter 1 introduces the background, motivations and contributions of our study. 
Chapter 2 introduces the Hadoop YARN, the current most popular open source job scheduler for big data processing frameworks and reviews the related work on efficiency-oriented scheduling, fair scheduling, latency-aware scheduling, energy-efficient scheduling, cost-effective scheduling, greenaware scheduling and multiple-objective optimizations.

Chapter 3 presents our study for the workload-aware scheduling for data intensive computing. We first develop a workload characteristic model with the regression approach to characterize the tradeoff between resource efficiency and fairness. Next, we build a workload-aware meta scheduler that adaptively optimizes the resource efficiency of the cluster given the user-defined threshold on fairness loss, or vice versa.

Chapter 4 presents a cost-effective and green-aware scheduler which is aware of joule efficiency to maximize the effectiveness of utilizing green energy as well as dynamic pricing.

Chapter 5 presents a general framework to support multiple-objective optimizations on geo-distributed data analytics systems.

Chapter 6 concludes our current studies and discusses the future work. 


\section{Chapter 2}

\section{Background and Related Work}

In this section, we introduce the background of job scheduling for big data processing frameworks and the related work.

\subsection{Hadoop YARN}

Hadoop YARN is the most popular open source job scheduler and resource mananger for the data analytics frameworks in the large-scale cluster. The system overview of Hadoop YARN [9] is shown in Figure 2.1. Hadoop YARN implements major responsibilities of resource management and job scheduling into separate components: Resource Manager and per-application App Master. The Resource Manager is the unified resource arbitrator among all applications in the system. The Apps Manager of Resource Manager launches an App Master for one application and it generates resource requests, negotiates resources from the Resource Manager and works with Node Managers to run and monitor the running tasks. Furthermore, Hadoop YARN provides fine-grained resource management instead of coarse-grained slot based manner. Each task represents its demand by a resource requirement vector which specifies the amount of different resources, e.g., $\langle 1 C P U, 3 G B\rangle$ indicates $1 \mathrm{CPU}$ core and 3 GB memory are needed by the task. YARN Scheduler of Resource Manager allocates the available resources reported by Node Manager to the pending tasks following a particular scheduling policy. 
There are four mainstream schedulers in Hadoop YARN, including First In First Out (FIFO) scheduler, Fair scheduler, Capacity scheduler and Dominant Resource Fairness (DRF) scheduler. The FIFO scheduler allocates the resources to applications in first-in-first-out sequence. The Fair scheduler is designed to fairly share the memory among all running users in large-scale multi-tenant clusters. The Capacity scheduler allows YARN applications to run in the multi-tenant cluster and maximizes the throughput of the cluster. It can be considered as a weighted Fair scheduler. DRF scheduler provides the fair allocation of multiple resources. Besides these schedulers, it is quite easy to integrate new schedulers into Hadoop YARN by implementing the YARN scheduling interface. Current schedulers in Hadoop YARN focus on individual objective. However, they do not consider the tradeoff between the resource efficiency, job latency, fairness and energy consumption. Therefore, new schedulers $[16,17,18,19]$ are proposed to address the tradeoff between these discordant factors.

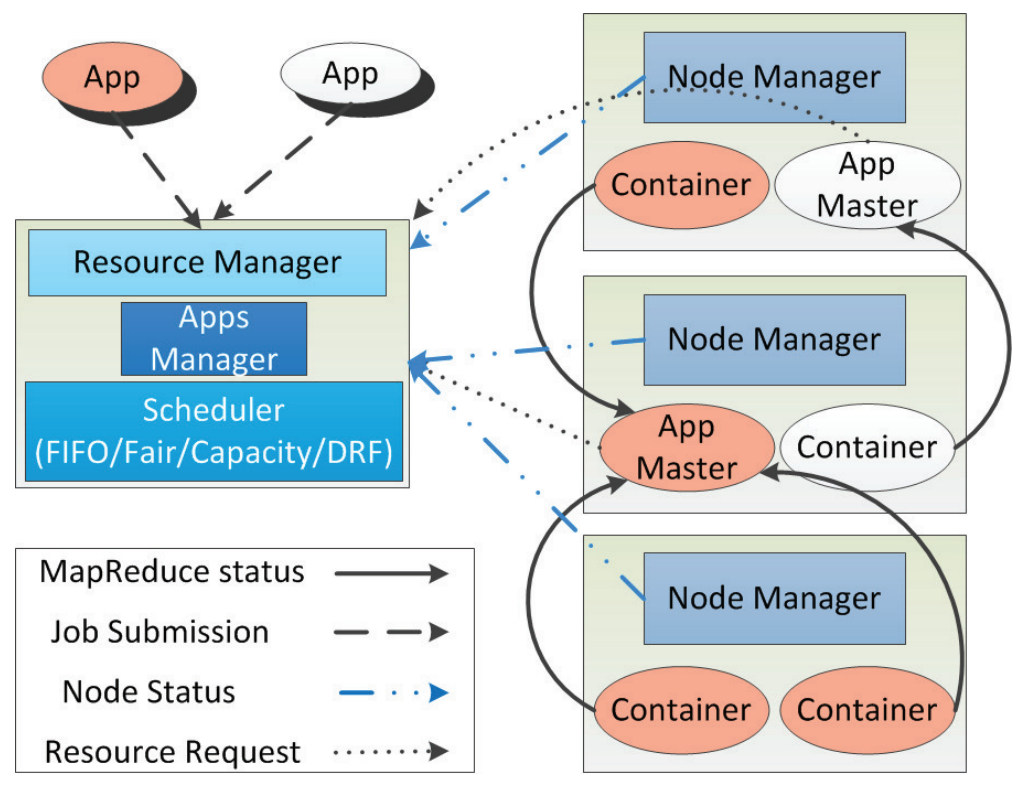

Figure 2.1: The overview of Hadoop YARN 


\subsection{Efficiency-oriented Scheduling}

Maximizing resource efficiency of Hadoop cluster is very important. In early years, the early generation of Hadoop abstracts resources as map/reduce slots and allocates the free slots among jobs. DynMR [20] implements more fine-grained reduce tasks with decoupled functional phases in order to resolve the low utilization problem caused by the data skew. RAS [21] captures the heterogeneous resource requirements of workload and dynamically adjusts slots on each machine to maximize the cluster utilization. ILA [22] improves the throughput of the virtual MapReduce clusters by considering the interference between map/reduce tasks. As the development of Hadoop, resource managers in the large-scale cluster are proposed to allocate the resources to the workload in a fine-grained way $[8,9,23]$. They provide a general approach to increase the resource utilization of the cluster by performing coordinated resource allocation and assignment. As the explosive growth of data, I/O optimizations become the core concern of data intensive applications. Delay scheduling gains nearly optimal data locality by only waiting a small of amount of time [24]. CoHadoop [25] explores more flexible data placement policy to improve the data locality. In addition to these, many more new I/O scheduling algorithms for MapReduce are proposed [26, 27, 28, 29]. These studies on Hadoop effectively increase the utilization of different kinds of resources in the large-scale cluster. However, few of them consider other objectives like fairness and energy consumption of the cluster and none of them study the relationship between the resource efficiency and other objectives.

\subsection{Fair Scheduling}

Fair scheduler [30] is proposed in the early generation of Hadoop to allocate slots fairly among different users based on the max-min fairness. Quincy [31] resolves fair allocations efficiently by mapping the fair scheduling problem as min-cost flow problem. Choosy [32] is a fair scheduler that considers the fairness with resource constraints in data centers. LTRF [33] resolves the fairness problem in a 
pay-as-you-go environment by considering the historical allocations. Except for the single-resource fair allocation mentioned above, there are a lot of studies for multi-resource fair allocation. Dominant Resource Fairness [13] is the first work to generalize the max-min fairness to multiple resources on Hadoop YARN. Wang et al. [34] extend Dominant Resource Fairness especially for the heterogeneous platform. Liu et al. [35, 36] propose a novel resource allocation approach, named Reciprocal Resource Fairness, in order to enable fair sharing multiple types of resources in the cloud. Even though many fairness definitions are proposed under different scenarios, they do not consider the relationship between fairness and other objectives like resource efficiency and job latency and none of them study the impact of different fairness definitions on the relationships of different objectives.

\subsection{Latency-aware Scheduling}

Job latency indicates the job completion time which is a critical factor application users care about. In single datacenter, many studies are proposed to reduce the job latency. Piranha reduces the job latency by optimizing short jobs in the cluster without affecting the larger jobs [12]. Spark efficiently accelerates the data-processing by utilizing fault-tolerant in-memory computing [37]. In multiple datacenters, Pu et al. [38] proposed to optimize the performance of geo-distributed data analytics by considering the heterogeneous Internet bandwidths to optimize data and task placement specially for MapReduce and Spark. SWAG reduces average job completion time with a novel job scheduling algorithms that coordinate job scheduling across datacenters with low overhead [39]. These studies do not consider the relationship between job latency and other objectives and the optimizations with multiple objectives simultaneously have been overlooked.

\subsection{Energy-efficient Scheduling}

Recently, there have been many research studies on optimizing the energy efficiency of MapReduce/Hadoop. Roughly, we can categorize the related work in the following three categories. 
The first category is to turn down some machines in the cluster for energy saving. Different approaches have been developed to identify the machines to turn down [40, 41]. As MapReduce colocates computation and storage in the same server, a main issue is the placement of data replicas in HDFS for data availability. Many energy-aware replication strategies are proposed [42, 43, 44].

The second category is to reduce the energy consumption according to the non-power proportionality of data centers. Lang and Patel [45] proposed an All-In Strategy (AIS) approach, and run a batch of workloads with all the machines in the cluster and then powered down the whole cluster. BEEMR [46] splits the cluster into two disjoint zones, namely batch zone and interactive zone. The batch zone runs workloads in a batch fashion, and is turned into the low power state after the batch is finished. The interactive zone consists of a small, fixed fraction of cluster resources and is always powered on.

The third category is to leverage more energy-efficient hardware. Wimpy nodes (machines with low capability and low power consumption) have attracted considerate interests. The tradeoff on performance and energy consumption by wimpy nodes and normal servers have been explored for MapReduce (e.g., [45, 47]).

These energy-efficient optimizations effectively reduce the total amount of energy consumption of the large-scale cluster. However, they do not consider the energy efficiency of the workload that can be explored in a more fine-grained way to further reduce the energy consumption of the cluster.

\subsection{Cost-effective Scheduling}

The monetary cost becomes the huge burgen of the large-scale data center because of the large amount of energy consumption. Many online optimization algorithms are proposed to reduce the monetary cost in a single data center in the presence of workload and pricing uncertainties. Rahul et al [48]. developed an online control mechanism to minimize the electricity cost of the data center without any prior information on the workload and the pricing. Deng et al. [49] propose SmartDPSS, which 
smartly schedules energy from multi-source grid in a cost minimizing fashion. As large organizations need to operate multiple data centers, research has focused on the cost-effective energy management across multiple data centers. Qureshi et al [50]. leveraged the temporal and geographic variation of electricity prices to cut the electric bill for large-scale service. Le et al. [51] proposed to optimize the electricity cost of the data centers through intelligently distributing the computational workload among multiple data centers. These studies leverage the variant of the electric price in terms of temporal and spatial aspects to effectively reduce the monetary cost. However, its impacts on the resource efficiency and the fairness are not studied.

\subsection{Green-aware Scheduling}

There has been much fruitful research on non-green-aware algorithms and systems for deadlineconstrained job/task scheduling (e.g, [52]). Due to the dynamic feature of green energy, green energy aware computing becomes a hot research topic in data centers. Some studies have developed models and algorithms for predictions in order to minimize the cost of a data center. Chen et al. [53] schedule workloads effectively to utilize the renewable energy across geographically distributed datacenters. Preemption policies have been implemented on YARN $[54,55]$, which further promotes the elasticity of green-aware algorithms. However, checkpointing causes further I/O contention and preemption increases complexity of schedulers, needs further study.

Recently, a number of real green-aware systems are developed for different workloads. Researchers have tried to schedule batch jobs in order to maximize the usage of renewable energy [56, 57, 58]. Similar goals and techniques have been studied for interactive applications [59, 60, 61, 62]. For data centers that run both interactive and batch workloads together, Aksanli et al. proposed to adapt the amount of batch processing dynamically [58]. Goiri et al. has carried out a series of studies and a green data center prototype $[63,64]$ to manage deferrable and non-deferrable workloads at the presence of renewable energy. Chen et al. [65] proposed ReinDB, a novel database system supplied 
by renewable energy on a single server. Li et al. [66] utilize onsite green energy efficiently with data center power demand shaping technique. Some researchers leverage battery to store renewable energy $[67,68]$.

Few of the previous studies have paid attention to the joule efficiency problem, particularly in the MapReduce/Hadoop cluster powered with green energy. GreenPar [69] considers joule efficiency of the usage of green energy in the datacenters. However, it does not study the impact of on-peak/offpeak pricing on the cost in MapReduce/Hadoop cluster.

\subsection{Multi-objective Optimizations in Job Scheduling}

To the best of our knowledge, few studies consider the tradeoff between the resource efficiency, job latency, fairness and energy consumption for the job scheduling of the big data processing frameworks. Joe-Wong et al. [70] theoretically analyze the efficiency-fairness tradeoff with multiple resources for two families of fairness functions. Wang et al. [71, 72] analyze the tradeoff in multi-resource packet processing. Tetris [73] is the first work to explore the tradeoff between resource efficiency and fairness over YARN framework. Tetris leverages many alignment heuristics to efficiently pack tasks with heterogeneous demands to machines. Although these studies have observed the tradeoff between resource efficiency and fairness, they are not aware of the variation of the multi-resource demand of the running workload and still perform the scheduling with a static approach during the whole computation. ClavisMO is a modern multi-objective solver designed for both MapReduce and HPC clusters by deciding the most suitable job placement in a cluster. CARBYNE significantly improves application-level performance and cluster utilization while providing the same level of performance isolation as modern schedulers [74]. Graphene packs the complex DAGs efficiently and trade-offs fairness for faster job completion [75]. These studies only consider the single DC scenario and do not study the multi-objective optimization on the geo-distributed data analytics systems. 


\subsection{Summary}

Many studies mainly focus on optimization of the individual object. Even a few studies observe the tradeoff between different objectives. However, they do not perform detailed studies to explore the factors which impact these tradeoffs and address the tradeoff with some coarse-grained approaches. In addition to it, a general multi-objective optimizer which considers the resource efficiency, job latency, fairness and energy consumption especially for the big data processing framework is lacked. In comparison with the previous studies, we perform detailed studies to explore what factors and how they impact the resource efficiency, job latency, fairness and energy consumption of the data processing frameworks. Then, we propose some fine-grained approaches (workload-aware, costeffective, green-aware) to perform bi-criteria optimization to address the tradeoff. Eventually, we develop an efficient job scheduler which supports multiple-objective optimizations on geo-distributed data analytics systems. 


\section{Chapter 3}

\section{An Adaptive Efficiency-Fairness Meta-scheduler for Data-Intensive Computing}

In this chapter, we present our study for the workload-aware meta scheduling for data intensive computing.

\subsection{Introduction}

Recent studies have showed that there is a tradeoff between resource efficiency and fairness due to the resource contention and proposed some bi-criteria optimization algorithms [71, 70, 73, 72]. However, all of these algorithms are heuristics, which fail to address the tradeoff between resource efficiency and fairness. The reason is that, 1) the resource efficiency and fairness of different schedulers vary a lot due to their distinct optimization purposes; 2) due to the heterogeneous resource demands of submitted jobs, a static scheduler cannot always achieve the optimal tradeoff between the resource efficiency and fairness with the variation of the resource demands of running workload during the computation.

Figure 3.1 shows a multi-resource usage profile of tasks from Google in a data center of 12 thousands of machines based on Google trace [2]. Multi-resource means that the resource allocation is 
performed in multiple resource types (here, CPU and memory are the two resource types). The position of a circle indicates the CPU and memory resources acquired by tasks. The size of a circle is logarithmic to the number of tasks in this position. It shows that there are significantly heterogeneous demands for tasks on CPU and memory resources. Users have diverse demands on different resources and the most needed resource is called the dominant resource [13]. We define a metric (named complementary degree) to quantify the complementarity of the resource demands of the workload (see the formal definition in Section 3.2). Ideally, two workloads are complementary to each other, if they demand different dominant resources. For two workloads, the more complementary their resource demands, the greater the potential for resource efficiency optimization and the less the potential for the fairness loss sacrificed in the resource efficiency optimization. To illustrate that different complementary degrees of submitted jobs have significant impact on the resource efficiency and fairness in the sharing environment, we conduct an experiment with Google trace. Figure 3.2 shows the makespan reduction and the fairness loss of a efficiency-oriented scheduler for a workload with different complementary degrees (The detailed setup can be found in Section 3.4). With the increase of complementary degree, the makespan reduction becomes higher, and the fairness loss first increases significantly to a highest point and later decreases after it. When the complementary degree lies between 0.5 and 1 , the complementary degree increases, the makespan reduction is getting higher and the fairness loss is also getting larger. When the complementary degree lies between 1 and 2 , the complementary degree increases, the makespan reduction is still getting higher while the fairness loss is getting less. It shows that the resource efficiency and fairness of a scheduler are sensitive to the variation of the workload.

Therefore, we should develop a scheduler to be aware of the workload dynamics and efficiencyfairness tradeoff. Since researchers keep inventing new scheduling algorithms, a viable approach is to leverage existing efficiency- and/or fairness-optimized schedulers and develop a meta-scheduler to address the efficiency-fairness tradeoff. Particularly, we propose a meta scheduler called FLEX 


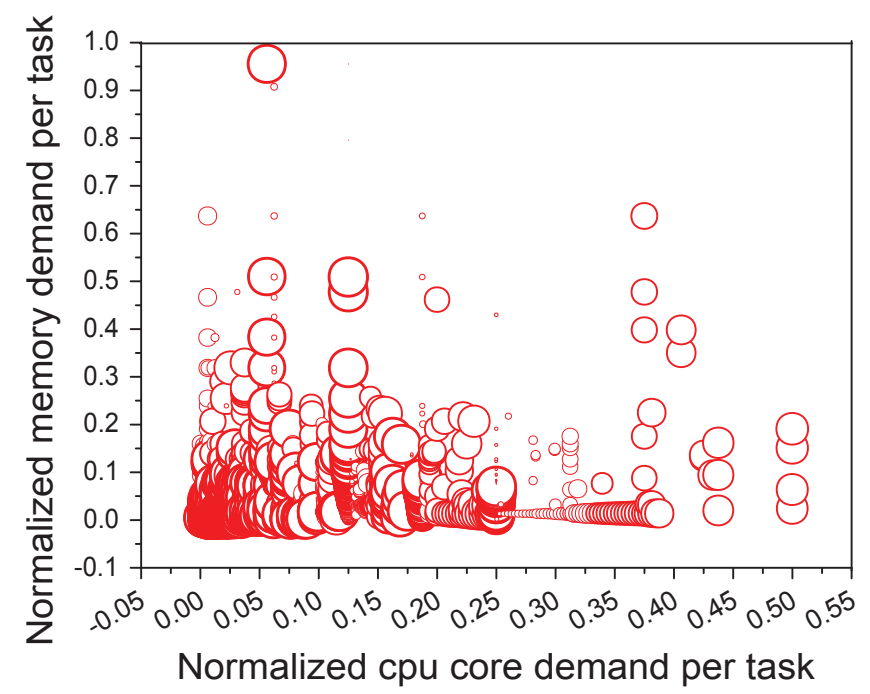

Figure 3.1: Heterogeneous resource demand for tasks from Google traces [2]

that takes advantage of existing schedulers in Hadoop YARN and adaptively chooses the most proper scheduler according to the current running workload and the user-defined SLA (Service Level Agreement). FLEX performs the bi-criteria optimization for resource efficiency and fairness. Given a predefined threshold on fairness loss (i.e., the maximum fairness loss the user can tolerant), FLEX is able to maximize the resource efficiency of the cluster, or vice versa. FLEX is highly extensible and allows adding/removing any new schedulers on Hadoop YARN. In our current implementation, FLEX supports all mainstream schedulers in the latest Hadoop YARN and one efficiency-oriented scheduler which applies the efficient task packing algorithm proposed in Tetris [73]. Equipping with these candidate schedulers, the FLEX leverages the machine-learning approach to adaptively choose the most proper scheduler with the variation of the current running workload and the user-defined SLA. To support adaptive scheduling, we model the scheduler choosing problem as a well-known classification problem, and resolve the classification problem with decision tree that mainly targets the multi-class classification. Firstly, we train the decision tree model with the data consisting of the scheduling result for different workloads and user-defined SLAs. Then, given the current running workload and user-defined SLA, the target scheduler can be easily inferred based on this built 


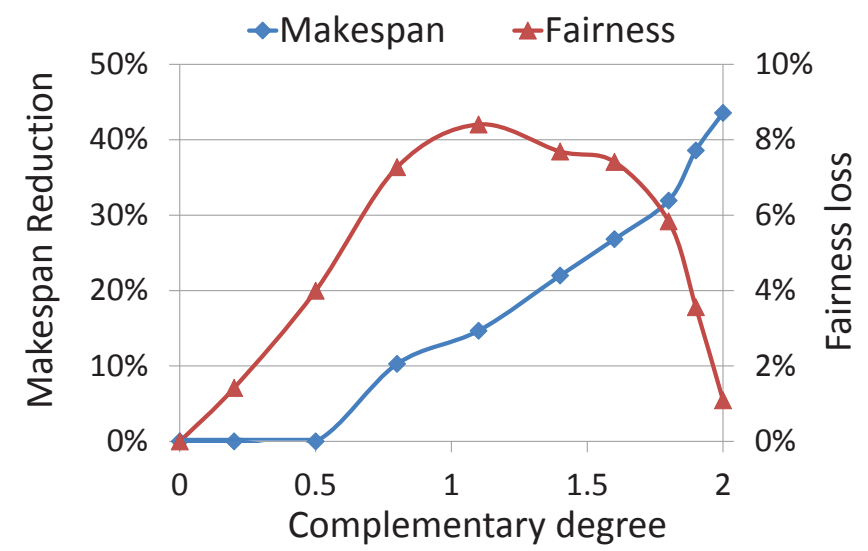

Figure 3.2: Tradeoff between resource efficiency and fairness for workloads with different complementary degrees

decision tree model.

We implement FLEX in Hadoop YARN (2.6.0). We conduct experiments with real deployment in a local cluster and perform simulation studies with production traces. FLEX outperforms than the state-of-the-art scheduling algorithm [73] in two aspects: 1) Given a predefined threshold on the fairness loss, FLEX reduces the makespan by up to $22 \%$ and $24 \%$ in real deployment and the large-scale simulation, respectively; 2) Given the predefined threshold on the makespan reduction, it reduces the fairness loss by up to $75 \%$ and $73 \%$ in real deployment and the large-scale simulation, respectively.

\subsection{Workload Characterization Model and Problem Statement}

The meta-scheduler adaptively adopts different candidate schedulers according to the characteristic of the workload and user-defined SLAs. To be aware of the variation of the workload, we propose a resource-based model to characterize the workload and optimize the resource efficiency and the fairness of the system by adaptively choosing the most proper scheduler at runtime. In this section, we first present our workload characterization model, and then formulate our optimization problem. 


\subsubsection{Workload Characterization Model}

We propose an entropy-based approach to calculate the complementary degree of the workload in this section.

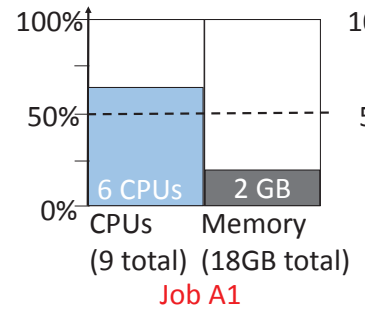

(a) Workload A
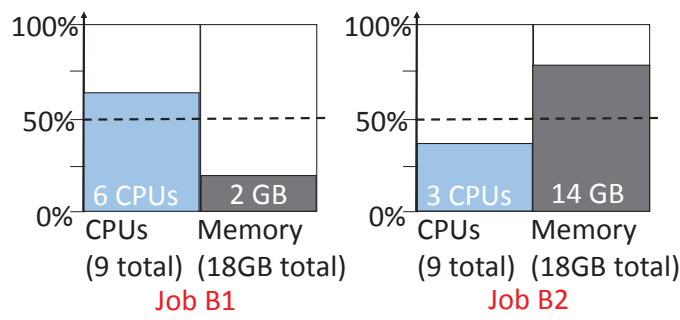

(b) Workload B

Figure 3.3: The workloads with different resource demands.

Entropy is mainly used in information theory to characterize the distribution of information content. Larger entropy indicates more random information. We treat the resource demand as the information and then utilize entropy to characterize the distribution of the resource demands of the workload. If the resource demands are randomly distributed, they are complementary for co-scheduling. The randomness of the resource demands indicates the degree of their complementarity. Therefore, we extend the definition of entropy in information theory to quantify the complementary degree of the resource demands of the workload.

We use an example to describe the basic concept of complementary degree. We consider two kinds of resources in our system: CPU and memory. Figure 3.3 shows two different workloads and each of them consists of two jobs. The job A1 and A2 in workload A are both CPU-intensive. The job B1 and B2 in workload B are CPU-intensive and memory-intensive, respectively. The resource demands of workload A are not complementary to each other as they are all CPU-intensive. In contrast, the resource demands of workload B are complementary to each other because their intensive resources are different. The complementary degree we defined below is used to quantify the complementarity of these resource demands. The complementary degree of the workload B should be higher than the complementary degree of the workload A. 
We define some terminology for a multi-resource allocation system. We consider $m$ types of hardware resources (e.g., CPU, memory, disk, network) denoted as $R=\{1, \ldots, m\}$. Let $U=\left\{u_{1}, \ldots, u_{n}\right\}$ be the set of $n$ users which share the cluster together. For each user $i$, let $D_{i}=\left(D_{i 1}, \ldots, D_{i m}\right)$ be the user's resource demand vector, in which $D_{i j}$ is the normalized amount of the resource $j$ needed by each task of user $i$ (normalized according to the capacity of the cluster). For simplicity, we only consider the running tasks and assume the demands for all users are non-negative, e.g., $D_{i j} \geqslant 0, \forall i \in U, j \in R$. We define the dominant resource of user $i$ as $k_{i}$ and it indicates the most needed resource type of user $i^{\prime} s$ tasks. $k_{i}$ is calculated as

$$
k_{i}=\underset{j \in R}{\arg \max } D_{i j}
$$

We then calculate the percentage of the users whose dominant resource type is $k$ as

$$
P(k)=\frac{\sum_{i=1}^{n} \delta\left(k_{i}, k\right)}{n}
$$

We use an indicator function $\delta(x, y)$ which is shown as

$$
\delta(x, y)= \begin{cases}1, & \text { if } x=y \\ 0, & \text { otherwise }\end{cases}
$$

Statistically, $P(k)$ is the probability of observing a job whose dominant resource type is $k$.

Based on the underlying probability distribution of jobs with different dominant resource types, we quantify the complementary degree of the workload with entropy. According to the definition of entropy [76], the complementary degree $d$ of the workload can be easily calculated as

$$
d=-\sum_{i \in R} P(i) \log _{2} P(i)
$$




\subsubsection{Problem Statement}

The workload characterization model captures the resource usage of different resource types, which essentially reflects the potential of efficiency-fairness tradeoff in the system. Due to the variation of the workload, we need a more fine-grained approach to realize the potential. Based on the workload characterization model, FLEX monitors the variation of the running workload and adaptively chooses the most proper scheduler to perform bi-criteria optimization for resource efficiency and fairness. In this work, we evaluate the resource efficiency with the makespan, e.g., the maximum execution time from the first task submitted till the lasted task completes. For the fairness, following the fairness concept of the DRF scheduler [13], we consider the fairness of dominant resource $k_{i}$ of user $i . k_{i}$ is calculated according to Equation 3.1. We assume that $R_{i}$ is the normalized total amount of obtained dominant resource of user $i$ (normalized according to the capacity of the cluster). We say the resources are allocated fairly among all users if $R_{1}=R_{2}=\ldots=R_{n}$. By following the evaluation approaches used in DRF and Tetris [73], we also evaluate the target scheduler with the improvement on the resource efficiency and the fairness loss compared with the DRF scheduler. We quantify the improvement on the resource efficiency with the percentage improvement (or reduction) on the makespan. Let the makespan under the target scheduler is $T^{*}$ and the makespan under DRF scheduler is $T$, then the makespan reduction $E$ of the target scheduler is

$$
E=\max \left\{\frac{T-T^{*}}{T}, 0\right\} .
$$

Many fairness definitions are proposed to guarantee the fair resource allocation in the shared environment $[13,73,33]$. As in the previous studies $[13,73]$, it is very natural and straightforward to measure the fairness along the lifetime of the job with its favored/degraded performance. In this work, we directly apply this kind of approach. For completeness, we also study the impact the other kind measurement approach which is defined from the resource usage's aspect [33] (more details can be referred in Section 3.4.2.4). 
Many previous studies (e.g., $[13,73])$ quantify the fairness from the per-user performance aspect because the execution time of the applications from individual users is the key factor that the users really care about in the shared cluster and the comparison of fairness can be made by considering how the schedulers favor/degrade performance among users (e.g., [73, 77, 78]). Particularly, most of those studies $[13,73]$ have measured the fairness of the proposed scheduler on the basic of slowdown of each user compared with that of a fair scheduler (such as DRF [13] scheduler and max-min fair scheduler [30]). More precisely, the slowdown refers to the difference in the expected running time for the same user between when it is scheduled with others under the proposed scheduler and when it is scheduled under a fair scheduler. We directly follow these studies and apply their definitions of fairness in this work. That is, we quantify the fairness loss of the target scheduler with the average slowdown (reduction) of the job finishing time compared with the fair scheduler. Note that, the data-processing application can be delayed by a fixed amount of time and the maximum tolerant slowdown is defined in the SLA of our optimization problem. The slowdown of the target scheduler cannot violate the user-defined SLA. We still assume $U=\left\{u_{1}, \ldots, u_{n}\right\}$ is the set of $n$ users who share the cluster together. For each user $i$, the completion time of its application under the target scheduler and fair scheduler are $t_{i}^{*}$ and $t_{i}$, respectively. Let $S_{i}$ be the reduction of the completion time of the application from user $i$ and $S_{i}$ can be easily calculated as

$$
S_{i}=\max \left\{\frac{t_{i}^{*}-t_{i}}{t_{i}}, 0\right\}
$$

Then the fairness loss $F$ of the target scheduler during the whole execution can be calculated as

$$
F=\frac{\sum_{i \in U} S_{i}}{n}
$$

Given the workload characterization model and the definition of resource efficiency as well as fairness, the optimization problem of this work is formulated as follows. We consider the bi-criteria 
optimization between resource efficiency and fairness in this work. Particularly, we study two cases for the bi-criteria optimization problem. Given a predefined threshold on the fairness loss $F$, which represents the maximum fairness loss the user can tolerant compared to fair scheduler, FLEX maximizes the makespan reduction $E$ of the system by adaptively choosing the most proper scheduler according to the characteristic of the workload. Similarly, Given the predefined threshold on the makespan reduction $E$, which indicates the makespan reduction expected by the user in comparison with the fair scheduler, FLEX minimizes the fairness loss $F$ of the system.

\subsection{System Design of Meta-scheduler}

We introduce the design and implementation of the meta-scheduler FLEX in this section. First, we list a number of rationales used in designing our meta-scheduler FLEX. Second, we give the system overview of FLEX. Third, we describe each component of FLEX in detail. Finally, we show the implementation of FLEX on Hadoop YARN.

\subsubsection{Rationale of System Design}

As we review the related work in Section 2, researchers keep inventing new schedulers for Hadoop/YARN. Ideally, any scheduler reflects some aspect of the efficiency-fairness tradeoff. Thus, a static approach based on heuristics (e.g., [24, 73]) cannot fully address the efficiency-fairness tradeoff. Motivated by the widely applicability of meta-scheduler design in different application environments [79, 80, 81], we propose to design a meta-scheduler to take advantage of the existing or future schedulers in Hadoop YARN. Our system design is driven by a number of rationales.

- Extendability. Since the system targets at a meta-scheduler design, it should be extensible to new schedulers besides the existing schedulers. FLEX should be able to adapt to the removal/addition of a scheduler. Note that, the effectiveness of meta-scheduling still depends on 
the set of candidate schedulers, and how they can cover the spectrum of the efficiency-fairness tradeoff. The design of our meta-scheduler is able to exploit the spectrum of the efficiencyfairness tradeoff exposed by the candidate schedulers.

- Workload variation awareness. Since the most proper scheduler depends on the characteristic of the workload, the system should provide an automatic mechanism to adaptively switch the scheduler when the workload varies.

- Lightweight runtime overhead. Since the decision has to be made at runtime in order to adapt to workload dynamics, the overhead of the scheduler should be lightweight.

\subsubsection{System Overview}

The overall design of our meta-scheduler FLEX is shown in Figure 3.4. FLEX integrates multiple existing schedulers in Hadoop YARN into a single aggregated view. These schedulers are candidate schedulers to be chosen by FLEX. FLEX performs the bi-criteria optimization between the resource efficiency and fairness. Given the user-defined threshold on the fairness loss, the scheduler which satisfies the user-defined SLA and maximizes the resource efficiency of the cluster is chosen, or vice versa. FLEX implements these optimizations through adaptive scheduling. As the resource efficiency and fairness of a scheduler depend on the workload, FLEX automatically detects the variation of the current running workload based on the workload characterization model and leverages the machine learning approach to adaptively select the most suitable scheduler from all candidate schedulers. The target scheduler selected from all candidate schedulers becomes the current scheduler of Hadoop YARN and is responsible for the resource allocation till another scheduler is chosen. When the resources in the cluster become available, the current scheduler of Hadoop YARN allocates the resources to the pending jobs/tasks according to its scheduling policy and launches them on the corresponding machine in the cluster. 


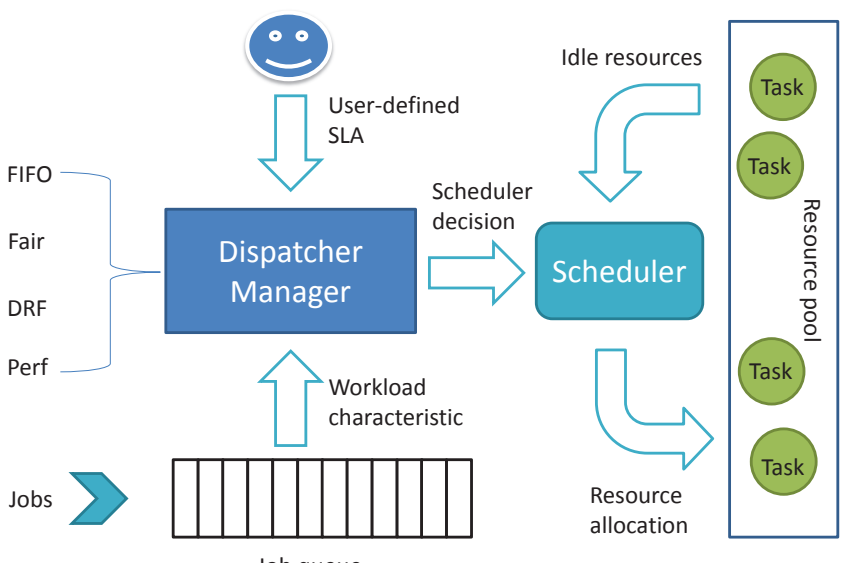

Figure 3.4: Design of the meta-scheduler FLEX

\subsubsection{Detailed Design of FLEX}

We introduce the design of FLEX in detail. FLEX consists of a number of candidate schedulers, a dispatcher manager and a scheduler switcher. FLEX integrates existing mainstream schedulers in Hadoop YARN and allows the addition of new schedulers. The dispatcher manager utilizes the machine learning approach to adaptively decide the most suitable scheduler from all candidate schedulers according to the workload characteristics and the user-defined SLAs. Once the target scheduler is decided, the scheduler switcher updates the current scheduler of Hadoop YARN and the chosen scheduler is responsible for the resource allocation till another scheduler is chosen.

Candidate schedulers. Essentially, the application scheduling in Hadoop YARN is actually a procedure of determining the job/task execution order of all running applications. All applications are added into a queue and Hadoop YARN determines their execution order according to the policy of current scheduler when resource becomes available. Resource requests for the application in the head of the queue are granted first; once all requests of the application have been satisfied, the next application in the queue is scheduled, and so on. The effectiveness of meta-scheduling in addressing the efficiency-fairness tradeoff depends on the set of candidate schedulers and how they can cover the spectrum of the efficiency-fairness tradeoff. In our current implementation, we consider three 
mainstream schedulers in Hadoop YARN and add one efficiency-oriented scheduler in our system to demonstrate that new candidate schedulers can be added in a flexible manner.

- FIFO scheduler sorts all applications in the order of submission (first in, first out).

- Fair scheduler considers the memory usage of all applications and sorts these applications in the decreasing order of how far they are from their fair memory shares.

- DRF scheduler takes the amount of dominant resource as the fair share in a system supporting multiple resource types and sorts the applications in the decreasing order of how far they are from their fair shares. Basically, Hadoop YARN has implemented the policy in the original paper [13].

- Perf scheduler is an efficiency-oriented scheduler which supports makespan-aware job packing algorithm which is proposed in Tetris [73]. It sorts all applications in the decreasing order of the similarity between their resource requests and the available resources.

Dispatcher manager. With the variation of the running workload and the user-defined SLA, FLEX needs to adaptively choose the most suitable scheduler from all candidate schedulers. We model this decision as a classification problem in the machine learning area. All available candidate schedulers are a finite set of class labels and the decision of the target scheduler is actually a process of identifying a class label for the target scheduler. Decision tree [82], a well known predictive modeling approach used in machine learning, fits our problem quite well. It creates a tree-structured predictive model which estimates the class of a target item based on the observed features about the item. We can use different metrics for measuring the splits of the set of items to be classified. These metrics quantify the homogeneity of the target label within the subsets. We use two kinds of metrics called Gini impurity and Information gain in our system. Gini impurity measures how often a randomly selected item from the set would be incorrectly labeled if it was labeled randomly based 
on the distribution of labels [83]. Information gain is based information theory and the information gain of an event if the discrepancy of the amount of information before observing that event and the amount after observation [83].

The decision tree for the optimization problem can be trained from the labeled training dataset. The training item is in the format of $(d, s, Y) . d$ is the complementary degree of the workload calculated according to Equation (3.4). The SLA $s$ is the user-defined threshold on the fairness loss or the makespan reduction. $Y$ is the target scheduler we will classify. $d$ and $s$ are the features of data and the $Y$ is the label of the data. During the training phase, the variable $Y$ is already labeled. If the goal is to optimize the resource efficiency of the cluster given the user-defined threshold on the fairness loss, then $Y$ is the scheduler which satisfies the user's requirement and maximizes the makespan reduction. Similarly, if the goal is to optimize the fairness among all jobs given the user-defined threshold on the makespan reduction, then $Y$ is the scheduler which can achieve the best fairness under the constraints on resource efficiency. Given the training dataset, we utilize a common used heuristic approach [84] to train the decision tree offline and uses it to infer the type of $Y$ at runtime according to the workload characteristic $d$ and the user-defined SLA $s$. One example of the decision tree is shown in Figure 3.5. The brunch nodes are shown with solid rectangles and the condition expressions inner these rectangles are the rules used for classification according to features of the training item. In this example, we mainly consider two features: the complementary degree of the resource demands and the userdefined threshold on the fairness loss. The leaf nodes which are shown with dotted rectangles indicate the classification results. The "Samples" is the total number of items and the vector shows the number of the different labels for these training items. The label with the highest number indicates the label of the leaf node which is used for referring. Here, the label is the target scheduler type. In inferring phase, give on data item, we can easily find its corresponding leaf node by searching on the decision tree according to the features of the data item and obtain its label.

We generate the training dataset with the following three steps. First, we synthesize a set of workload with different resource demands. Second, we specify different SLAs according to the operators' 


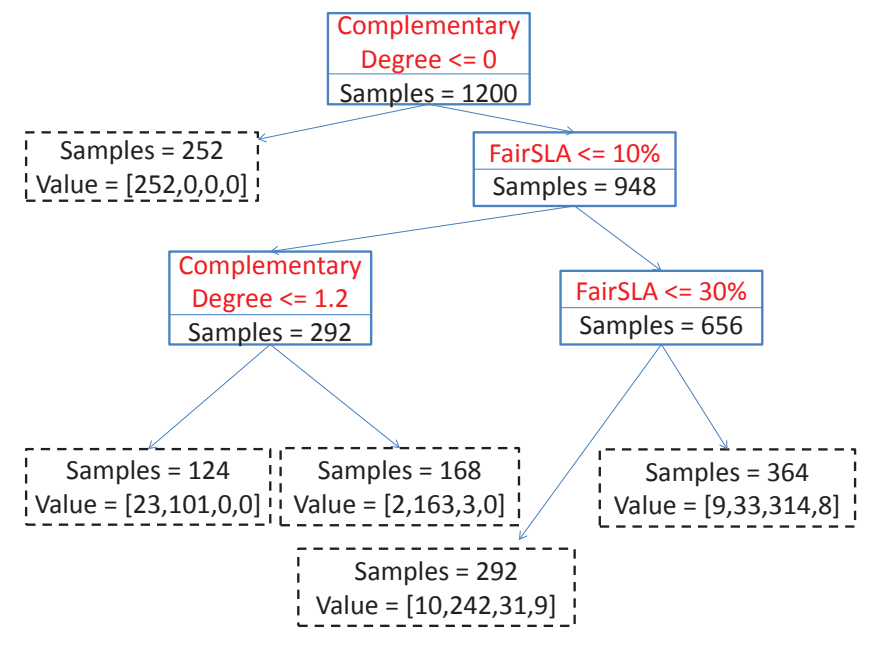

Figure 3.5: An example decision tree trained with the default parameter setting

requirements. Third, for each combination of the workload and the SLA, we run the workload with different schedulers and obtain the performance and fairness. The scheduler that achieves the best optimization for the goal while satisfying the SLA is labeled as the target scheduler type. We then calculate the complementary degree of the current workload and gain one training item consists of complementary degree, the corresponding SLA and the target scheduler type. We repeat this process for all combinations of the workload and the SLA to gain all training dataset.

Based on the decision tree, the dispatcher manager adaptively decides the most suitable scheduler for Hadoop YARN at runtime. The detail of the decision procedure is shown in Algorithm 2. The scheduler manager calculates the complementary degree when new jobs come or existing jobs finish. Once the complementary degree changes, then we predict the type of the target scheduler among candidate schedulers with the decision tree and update the current YARN scheduler with the decided scheduler. This scheduler is responsible for the resource allocation in Hadoop YARN till another new scheduler is chosen. The time complexity of our scheduling algorithm is decided by the inference time complexity of the decision tree which is linear to the hight of the tree. Thus, if $h$ is the height of the trained decision tree, then the time complexity of the scheduling algorithm is $O(h)$. 


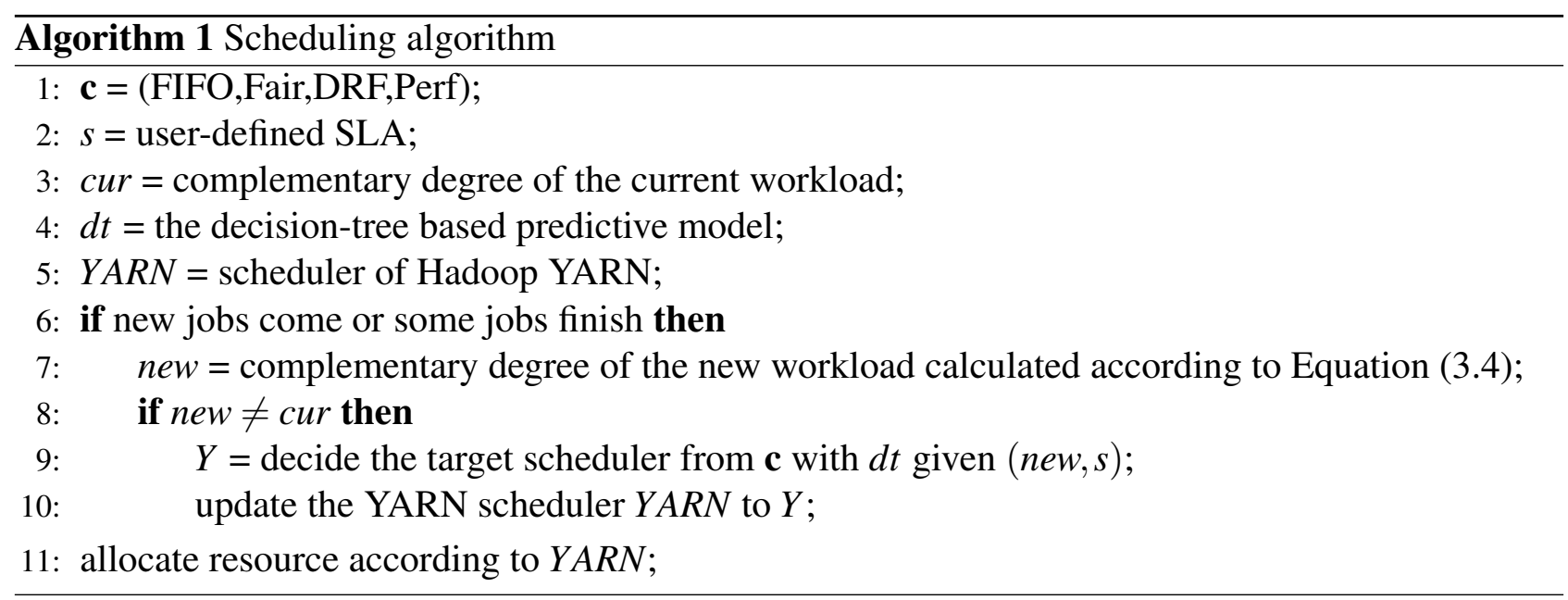

Scheduler switcher. In order to support the adaptive scheduling of FLEX, we implements a scheduler switcher that is able to dynamically change the scheduling policy of Hadoop YARN at runtime. Once the target scheduler is chosen from the candidate schedulers by the dispatcher manager, FLEX replaces the current scheduler of Hadoop YARN with the chosen scheduler and this new scheduler is responsible for the resource allocation of Hadoop YARN till another scheduler is chosen.

\subsubsection{Implementation on Hadoop YARN}

We incorporate FLEX into Hadoop YARN (2.6.0) by modifying Resource Manager of Hadoop YARN. The implementation detail is shown in Figure 3.6. In order to reduce the scheduling latency, Hadoop YARN applies the asynchronous event-based programming model. AsyncDispatcher is the core component of the asynchronous programming model. All components of Resource Manager need to register their events dispatchers in the AsyncDispatcher and communicate with each other by sending their events. AsyncDispatcher monitors all coming events and transfers each received event to the corresponding event dispatcher. We incorporate FLEX into YARN framework by making the following modifications:

- AppsManager provides a workload query API for other components to gain the information of current running workload including the input data, the application executable, the submission 
parameters and the resource demand of tasks. When a new job is coming, AppsManager notifies the other components by sending an event AppsEvent.Start to AsyncDispatcher. AsyncDispatcher notices WorkloadMonitor that a new application starts by sending an event MonitorEvent.AppStarted to it. If this application is completely new, AsyncDispatcher tells ModelTrainer by sending an event TrainerEvent.NewApp to it. When a job finishes, AppsManager tells the other components by sending an event AppsEvent.Start to AsyncDispatcher. AsyncDispatcher then notices WorkloadMonitor by sending an event MonitorEvent.AppFinished to it.

- Two new components, namely, ModelTrainer and WorkloadMonitor are integrated into Resource Manager of YARN. Their corresponding event dispatchers are firstly registered in AsyncDispatcher and listen to the corresponding events. ModelTrainer trains the workload characterization model in an offline model using decision tree method. It listens for the TrainerEvent.NewApp event and collects the information of the newly coming application from AppsManager. The ModelTrainer retrains and maintains workload characterization model periodically with the latest workloads. In general, the complexity of the decision tree training is $O(m n l o g n)$, where $m$ is the number of features and the $n$ is the number of samples in the dataset. In our experiment, the training time of a workload consisting of 1200 jobs is only 1 second, which is ignorable for data-intensive computing. On the other hand, this model training and maintainence can be in an asynchronous/offline manner, which does not interfere the execution of our meta-scheduler. WorkloadMonitor monitors the starting and finishing of all applications and notices DispatcherManager when their resource demands vary.

- We implement two new modules called DispatcherManager and SchedulerSwitcher in YARNScheduler component. All candidate schedulers (FIFO, Fair, DRF and Perf) are integrated into DispatcherManager. DispatcherManager listens for the SchedulerEvent.DemandVarying event and provides the adaptive scheduling based on the workload characterization model trained by 
ModelTrainer. SchedulerSwitcher supports the replacement of the scheduler of Hadoop YARN at runtime.

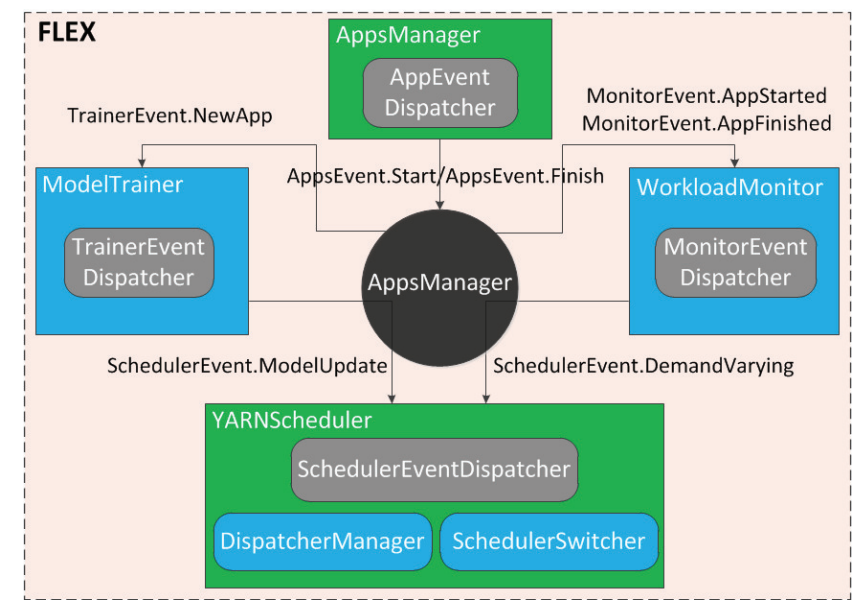

Figure 3.6: FLEX implementation on Hadoop YARN. The modified existing components are shown with green rectangles and the newly added components are shown with blue rectangles. Their corresponding event dispatchers are shown with grey round rectangles. The newly added modules of the component are shown with blue round rectangles.

\subsection{Evaluation}

In this section, we evaluate FLEX by extending Hadoop YARN in a small-scale cluster and in largescale simulator.

\subsubsection{Experiment Setup}

We evaluate FLEX through two sets of experiments. We evaluate FLEX by running our prototype implementation in our 10-nodes cluster. To evaluate the efficiency-fairness tradeoff and study the parameter impacts at large scale, we conduct a trace-driven simulations using the production trace in Google. 
Hadoop cluster. We use Hadoop YARN (2.6.0) and run the experiments in our local cluster. The local cluster consists of 10 machines, each with 12 CPU cores, 24GB memory and 500GB disk drivers. These machines are connected with $10 \mathrm{~Gb} / \mathrm{sec}$ Ethernet.

Workload. In our experiment, we use two synthesized workloads based on real traces from Facebook and Google.

- Facebook workload. We synthesize a Facebook-like workload according to the production trace (distribution of jobs sizes) [24]. This workload contains 100 jobs. Based on their resource demand, we divide them to 9 bins according to job type and job size which is shown in Table 3.1. It is consisted of many small jobs ( $1 \sim 15$ tasks) and a few large jobs (e.g., 800 tasks). The job interval is derived from Facebook production traces [85]. The demand distribution of map/reduce tasks is based on Figure 1 provided by Ghodsi et al [13]. As YARN currently only supports the allocation of CPU and memory, we also only consider these two resources in real cluster experiments and consider more types of resources in our trace-driven simulation. The actual jobs are generated from Hive benchmark [86] which contains four applications: grep, aggregation, ranking, and join.

- Google workload. We also synthesize a Google-like workload by randomly picking 100 jobs from Google trace over a one-hour period.

Metrics. We calculate the improvement on the resource efficiency and the fairness loss of the target scheduler compared with the DRF scheduler. To quantify the improvement on the resource efficiency, we use the percentage improvement (or reduction) on the makespan, illustrated in Equation 3.5. For the fairness loss, we calculate it with the average reduction of job completion times, illustrated in Equation 3.7. There are some other fairness measurement approaches which quantify the fairness from resource aspect [70, 72, 33]. For completeness, we also apply those kinds of approaches and achieve similar results which are shown in Section 3.4.2.4. In our experiments, we 
compare our proposed meta-scheduler FLEX with Tetris [73], the state-of-the-art scheduler which studies the tradeoff between the resource efficiency and the fairness in Hadoop YARN, by showing the reduction on the makespan and fairness loss of FLEX compared to Tetris.

To evaluate the accuracy of our decision tree model, we utilize the confusion matrix which is widely used to evaluate classification quality in machine learning through visualization [87]. Each column indicates the instances in a predicted class and each row of the matrix represents the instances in an actual class. The diagonal elements of the matrix represent the number of items for which the label is predicted correctly, while off-diagonal elements are those that are mis-predicated by the classifier. Higher diagonal values of the confusion matrix indicates better better prediction accuracy. Based on the confusion matrix, we use three important matrices called accuracy, recall and F1 score. The accuracy is the fraction of the total number of predictions that are correct. The recall is the proportion of the total number of actual instances that are predicted correctly. F1 score is a weighted average of the recall and prediction.

Trace-driven simulator. In order to evaluate FLEX at a larger cluster, we implement a tracedriven simulator that replays the production traces collected in Google cluster [2]. This trace provides the information of all tasks submitted by over 900 users on a cluster of about $12.5 \mathrm{k}$ machines in one month, including task submission times, execution time and normalized CPU/Memory/Disk resource demands. In order to accelerate the simulation, we simulate that 60 users are submitting tasks with different resource demands for three resource types (CPU, memory and disk) in 24 hours to a 600node cluster. We assume that all users share the cluster equally.

\subsubsection{Real Deployment Evaluations}

We evaluate the resource efficiency and fairness of FLEX with the synthetic workload in our local cluster. We compare FLEX with Tetris. First, we compare their makespan, fairness loss and resource utilizations. Then, we measure the overhead of our scheduling algorithm. Finally, we evaluate the tradeoff model used by FLEX with the cross-validation approach. 


\begin{tabular}{|c|c|c|c|c|c|c|}
\hline \multirow{2}{*}{ Bin } & \multirow{2}{*}{ Job Type } & \multicolumn{2}{|c|}{ Map Tasks } & \multicolumn{2}{|c|}{ Reduce Tasks } & \multirow{2}{*}{ \# Jobs } \\
\cline { 3 - 6 } & & $\#$ & Demand & $\#$ & Demand & \\
\hline \hline 1 & rankings & 1 & $<1,1 \mathrm{~GB}>$ & NA & NA & 38 \\
\hline 2 & grep & 2 & $<1,1.5 \mathrm{~GB}>$ & NA & NA & 18 \\
\hline 3 & aggregation & 10 & $<2,0.5 \mathrm{~GB}>$ & 2 & $<4,2 \mathrm{~GB}>$ & 14 \\
\hline 4 & rankings & 50 & $<4,1 \mathrm{~GB}>$ & NA & NA & 10 \\
\hline 5 & aggregation & 100 & $<2,1.5 \mathrm{~GB}>$ & 10 & $<2,2 \mathrm{~GB}>$ & 6 \\
\hline 6 & rankings & 200 & $<3,2 \mathrm{~GB}>$ & NA & NA & 6 \\
\hline 7 & grep & 400 & $<2,1 \mathrm{~GB}>$ & NA & NA & 4 \\
\hline 8 & join & 400 & $<1,2 \mathrm{~GB}>$ & 30 & $<2,0.5 \mathrm{~GB}>$ & 2 \\
\hline 9 & grep & 800 & $<2,0.5 \mathrm{~GB}>$ & 60 & $<1,3 \mathrm{~GB}>$ & 2 \\
\hline
\end{tabular}

Table 3.1: Job types and sizes for synthetic Facebook workloads.

\subsubsection{Overall Comparison}

We compare the makespan and the fairness loss for FLEX and Tetris. Figure 3.7(a) shows the makespan reduction of FLEX compared to Tetris for different thresholds on the fairness loss. The makespan reduction is up to $22 \%$ and $13 \%$ in average. This gain is achieved by considering the variation of the efficiency-fairness tradeoff during the computation. FLEX adaptively decides the suitable scheduler from the candidate schedulers according to the variation of the workload. Instead, Tetris applies the same scheduling policy (Perf) through the whole computation which loses many optimization opportunities for efficiency-fairness tradeoff. Similarly, FLEX significantly reduces the fairness loss compared to Tetris for different thresholds on the makespan reduction because FLEX skips the unworthy optimizations which would trade much unnecessary fairness loss for negligible makespan reduction. The result is shown in Figure 3.7(b). The reduction on the fairness loss is up to $75 \%$ and $59 \%$ in average compared to Tetris. By designing the adaptive scheduling algorithm, FLEX optimizes the resource efficiency as well as the fairness at the same time compared to Tetris. We also get the similar results with Google workload which are shown in Figure 3.8(a) and Figure 3.8(b). The main difference is that the reductions on the makespan and fairness loss for Google workload are both slightly smaller than that for Facebook workload because the resource demands of Google workload are more complementary than the resource demands of Facebook workload. 


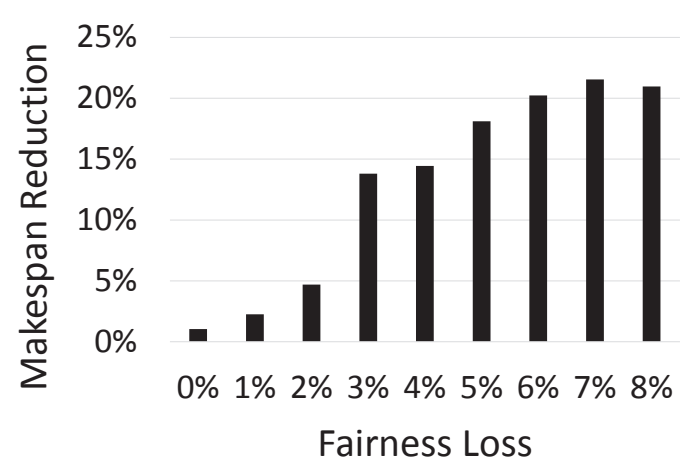

(a) Makespan reduction for different fairness (b) losses

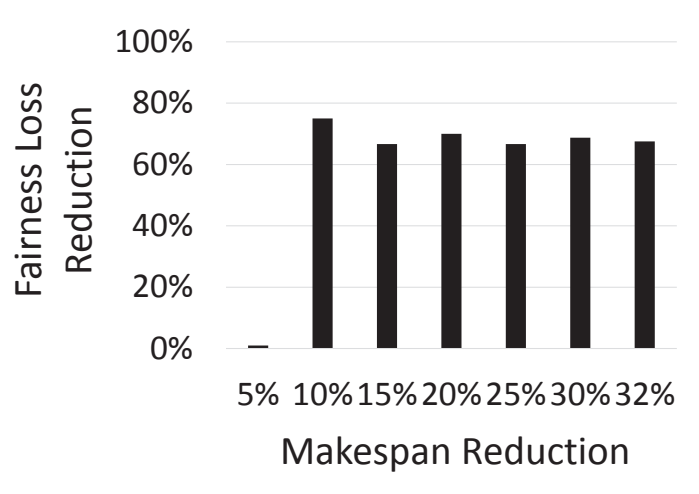

makespan reductions

Figure 3.7: The reduction on the makespan and fairness loss of FLEX compared to Tetris (Facebook workload)

To understand the improvement on the resource efficiency of FLEX compared with Tetris, we compare the resource utilization for both schedulers. As YARN currently only supports the allocation of memory and CPU, we show the utilization of memory and CPU for bother schedulers. In average, FLEX achieves $137 \%$ improvement on memory utilization and $122 \%$ improvement on CPU utilization. Figure 3.9(a) shows the detailed CPU utilization of both schedulers during the whole execution when the threshold on the fairness loss is set as $8 \%$. Similarly, the memory utilization of both schedulers is shown in Figure 3.9(b). The cluster is bottlenecked on different types of resources at different times and FLEX almost fully utilizes the bottlenecked resource all the time. In contrast, Tetris cannot fully utilize both resources due to a large number of resource fragmentation.

We conduct detailed studies on the impact of all candidate schedulers on the efficiency-fairness tradeoff in Figures 3.10-3.13 and have made the following major observations.

First, the schedulers have different optimization goals and the selection of the most suitable scheduler depends on the workload characteristics at runtime. For example, the scheduler which can gain the best resource efficiency changes with the variation of the workload and user-defined threshold on fairness loss. Figure 3.10 shows the selection distribution of all candidate schedulers for which can gain the best resource efficiency for different complementary degrees and different user-defined 


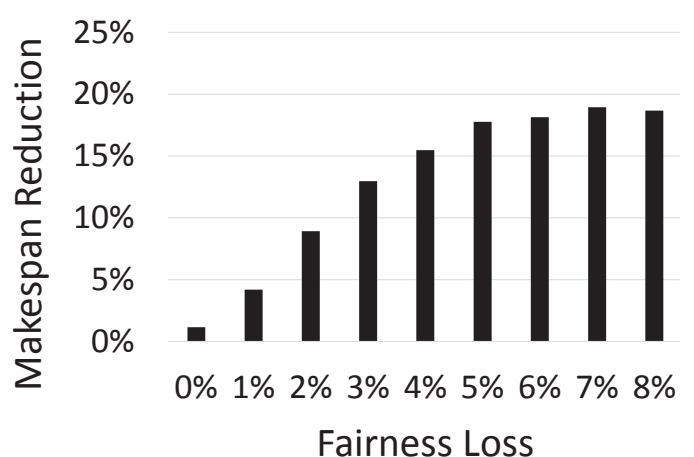

(a) Makespan reduction for different fairness losses

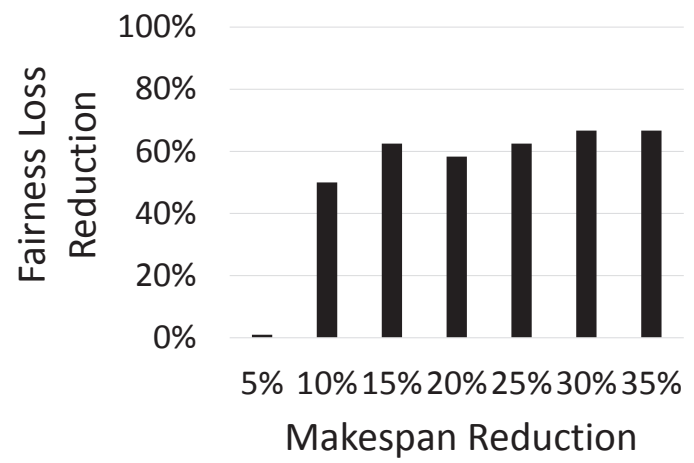

(b) Fairness loss reduction for different makespan reductions

Figure 3.8: The reduction on the makespan and fairness loss of FLEX compared to Tetris (Google workload)

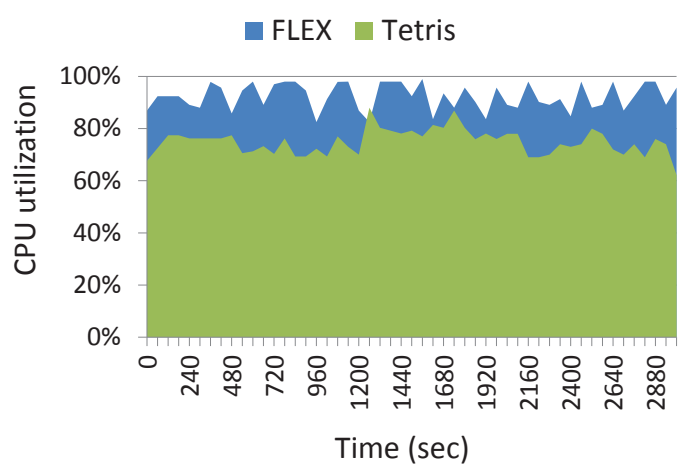

(a) CPU utilization

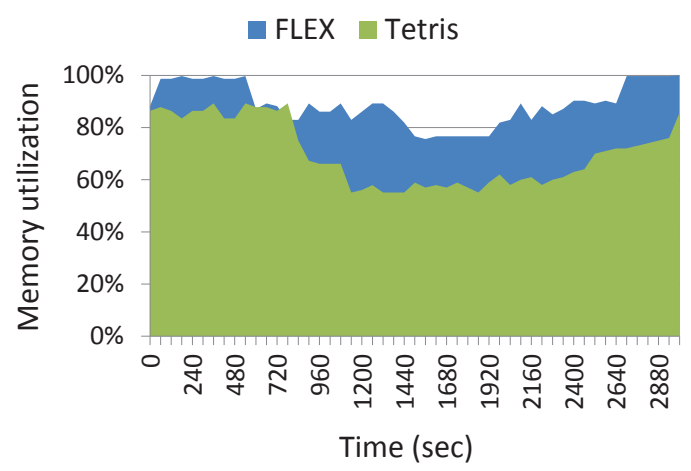

(b) Memory utilization

Figure 3.9: Resource utilization of the cluster under FLEX and Tetris during the execution

thresholds on fairness loss. DRF and Perf are mostly used than the other schedulers for all cases. It means that these two schedulers play very important roles in FLEX as we mainly study the efficiencyfairness tradeoff. With the increase of the complementary degree of the workload, the usage of Perf becomes even higher due to more optimizations which satisfies the user-defined SLA on the fairness can be conducted. Similarly, with the increase of the user-defined threshold on fairness loss, the usage of Perf also increases as more improvement on the resource efficiency can be achieved with the increase of user's tolerant on the fairness loss.

Second, the set of candidate schedulers is very important for the effectiveness of meta-scheduling. 


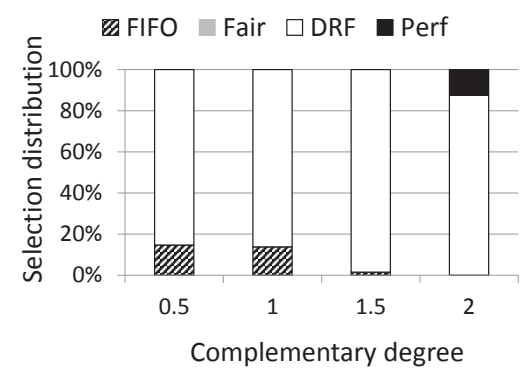

(a) Fairness loss $=0 \%$

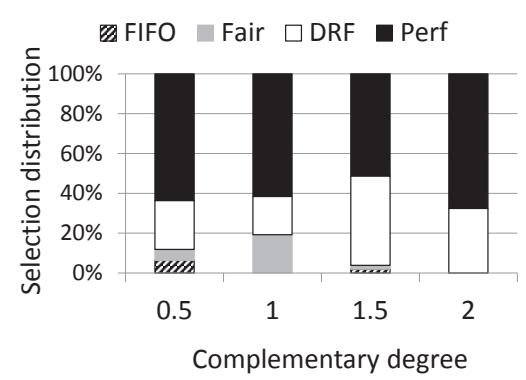

(d) Fairness loss $=30 \%$

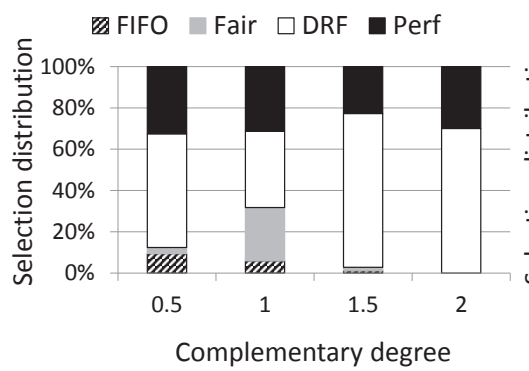

(b) Fairness loss $=10 \%$

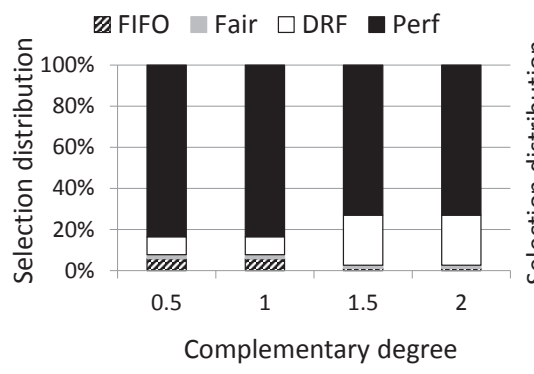

(e) Fairness loss $=40 \%$

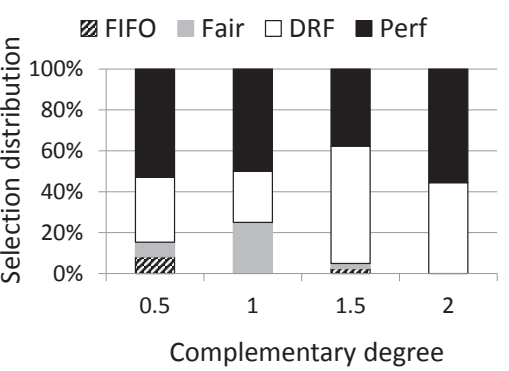

(c) Fairness loss $=20 \%$

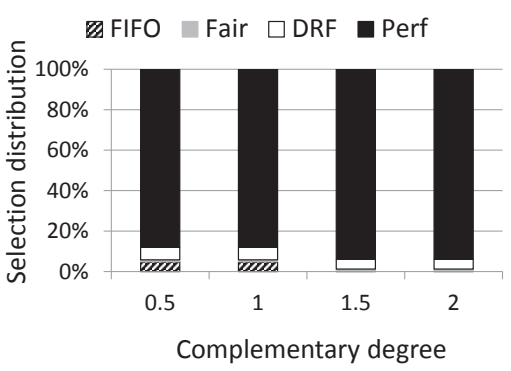

(f) Fairness loss $=50 \%$

Figure 3.10: The scheduler selection ratio

Our FLEX considers 4 candidate schedulers in the experiment. In order to show the impact of different combinations of schedulers on the effectiveness of the meta-scheduling, we enumerate all combinations of the candidate schedulers of FLEX and perform the scheduling for different workloads given different user-defined thresholds on fairness losses. We calculate the makespan reduction of each combination compared to DRF scheduler according to Equation 3.5. If the user-defined threshold on the fairness loss can not be satisfied no matter which scheduler is chosen, we give the penalty on resource efficiency by setting the makespan reduction to $-100 \%$. For each combination, we show the average makespan reduction given different thresholds on the fairness loss for all workloads. We classify these combinations into different categories according to the number of candidate schedulers which can be chosen.

- One scheduler. We assume only one scheduler can be selected. There are 4 combinations in total by choosing only one from these 4 candidate schedulers. Figure 3.11 shows their average 
makespan reduction given different thresholds on the fairness loss for all workloads. FIFO, Fair and DRF do not achieve any makespan reduction. For Perf, when the user-defined threshold on fairness loss is large enough, the makespan can be reduced.

- Tow schedulers. There are 6 combinations in total by choosing any two from these 4 candidate schedulers. Figure 3.12 shows their average makespan reduction given different thresholds on the fairness loss for all workloads. We can see that the combination of DRF and Perf outperforms all the other combinations. DRF guarantees that the user-defined threshold on the fairness can always be satisfied and Perf can reduce the makespan when the user's requirement is satisfied.

- Three schedulers. There are 4 combinations in total by choosing any three from these 4 candidate schedulers. Figure 3.13 shows their average makespan reduction given different thresholds on the fairness loss for all workloads. Combination (FIFO,DRF,Perf) and combination (Fair,DRF,Perf) achieve similar results and they both outperform than the other combinations. It further validates the conclusion observed before that DRF and Perf play very important role in our meta-scheduling.

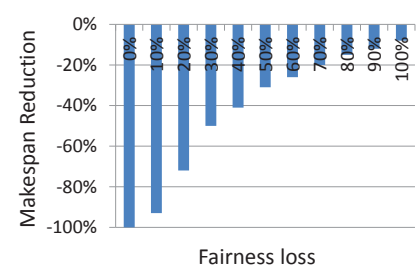

(a) FIFO scheduler

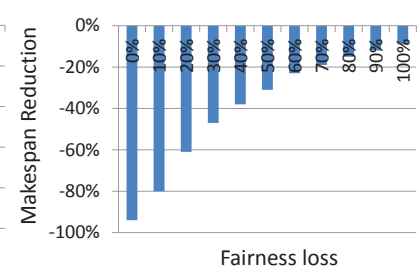

(b) Fair Scheduler

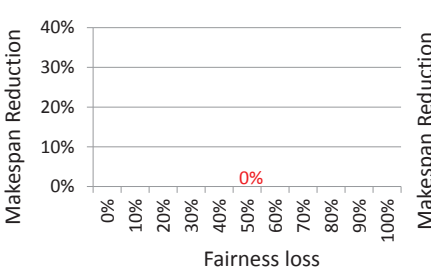

(c) DRF scheduler

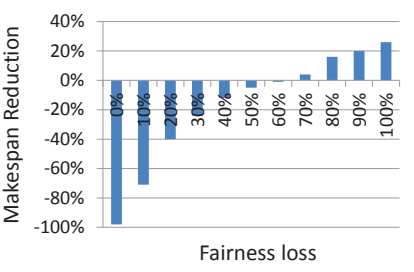

(d) Perf scheduler

Figure 3.11: One scheduler (4 combinations in total)

\subsubsection{Runtime Overhead Analysis}

In order to evaluate the runtime overhead of our scheduling algorithm, we run experiments with different numbers of jobs and tasks. We evaluate the scheduling overhead by observing the time needed 


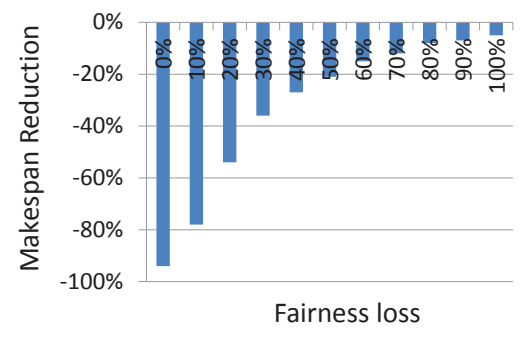

(a) FIFO + Fair

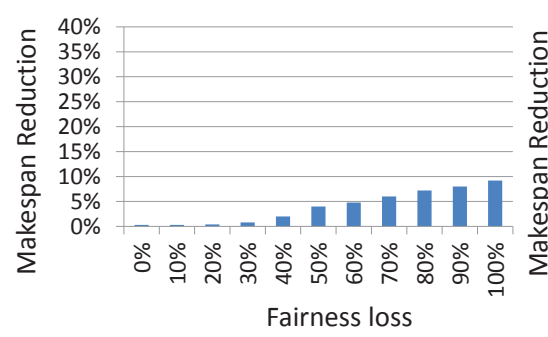

(d) Fair + DRF

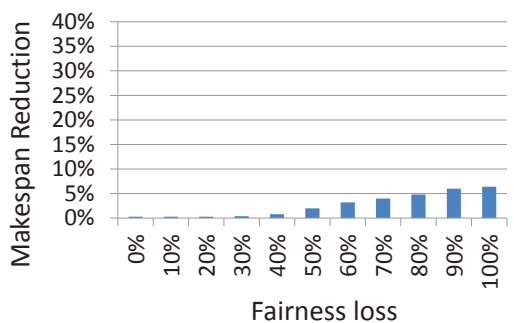

(b) FIFO + DRF

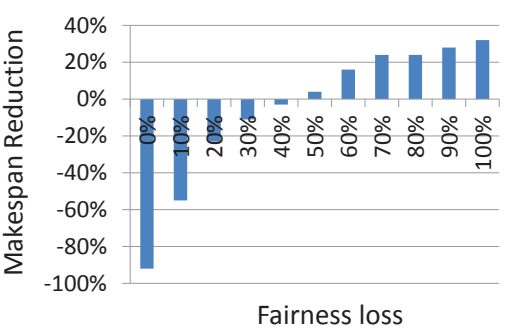

(e) Fair + Perf

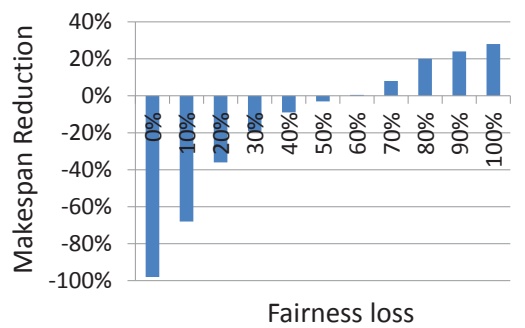

(c) FIFO + Perf

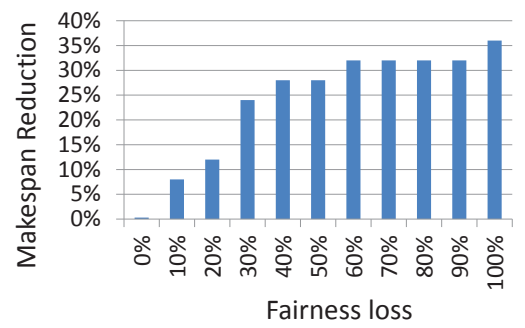

(f) DRF + Perf

Figure 3.12: Two schedulers (6 combinations in total)

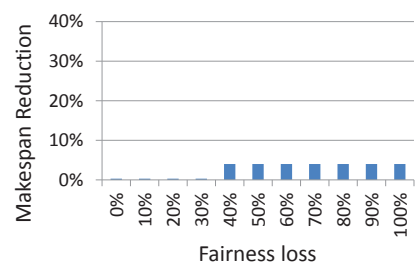

(a) FIFO + Fair + DRF

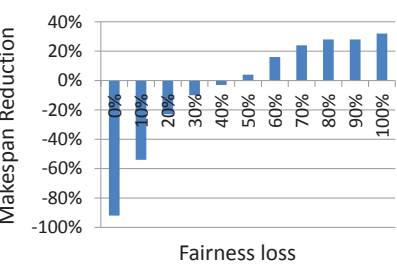

(b) FIFO + Fair + Perf

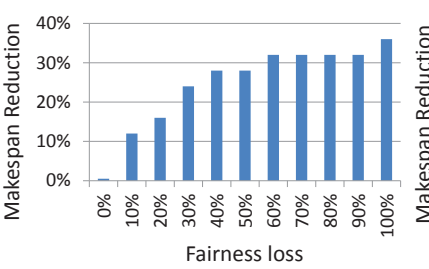

(c) $\mathrm{FIFO}+\mathrm{DRF}+$ Perf

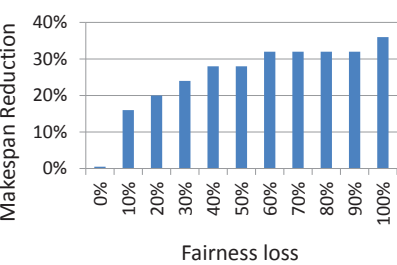

(d) Fair + DRF + Perf

Figure 3.13: Three schedulers (4 combinations in total)

by the Resource Manager (RM) to process the heartbeats coming from Application Masters (AM) and Node Managers (NM). YARN RM conducts the real resource allocation during the NM heartbeat and only updates the resource requests and responses during the AM heartbeat. The processing time of these heartbeats for different schedulers is shown in Table 3.2. For NM heartbeat, FLEX and Tetris are a bit slower than Hadoop Fair scheduler as they have more complex scheduling logic. For AM heartbeat, they all take the same time. All schedulers perform rather good scalability. We further evaluate the space overhead by monitoring the memory usage on Resource Manager and we find that FLEX consumes almost the same memory as Hadoop Fair scheduler. Our online algorithm design has 
little runtime overhead, rather than more complex optimizations based on linear programming [88].

\begin{tabular}{|l|l|l|l|}
\hline & Hadoop Fair scheduler & Tetris & FLEX \\
$10 \mathrm{~K}(50 \mathrm{~K})$ tasks & $10 \mathrm{~K}(50 \mathrm{~K})$ tasks & $10 \mathrm{~K}(50 \mathrm{~K})$ tasks \\
\hline NM heartbeat & $.05 \mathrm{~ms}(.18 \mathrm{~ms})$ & $.08 \mathrm{~ms}(.19 \mathrm{~ms})$ & $.08 \mathrm{~ms}(.20 \mathrm{~ms})$ \\
\hline AM heartbeat & $.04 \mathrm{~ms}(.04 \mathrm{~ms})$ & $.04 \mathrm{~ms}(.04 \mathrm{~ms})$ & $.04 \mathrm{~ms}(.04 \mathrm{~ms})$ \\
\hline
\end{tabular}

Table 3.2: Overheads: Average processing time of heartbeats from the Node Manager (NM) and the Application Master (AM) for different schedulers

\subsubsection{Model Evaluation}

We use grid search [89] to find the best settings for all the parameters of the decision tree. We apply these optimal parameter settings which are shown in Table 3.3 in the following experiments. Based on the optimal setting, we first evaluate the accuracy of the model with the cross validation approach and then study the impact of the prediction error on FLEX.

\begin{tabular}{|l|l|}
\hline Parameter & Best Value \\
\hline Criterion & "entropy" \\
\hline Splitter & "best" \\
\hline max-features & 2 \\
\hline max-depth & 3 \\
\hline min-samples-split & 10 \\
\hline min-samples-leaf & 5 \\
\hline max-leaf-node & 20 \\
\hline random-state & np.random \\
\hline
\end{tabular}

Table 3.3: Optimal parameter setting for the decision tree in the experiment

Cross validation with the same workload. We evaluate our decision-tree based tradeoff model with the cross validate approach which is widely used in machine learning. We shuffle the training data and split them into a pair of train and test sets. We use $70 \%$ data for training and validate the model with the remaining $30 \%$ data. With the parameter setting which is shown in Table 3.3, we firstly evaluate the accuracy of our decision tree with Facebook workload. The confusion matrix is shown in Figure 3.14 and the darker color in the diagonal of the confusion matrix indicates higher accuracy. We also show the detail of related metrics in Figure 3.15. The results show that our model is accurate enough and can effectively guide the adaptive scheduling of FLEX. 


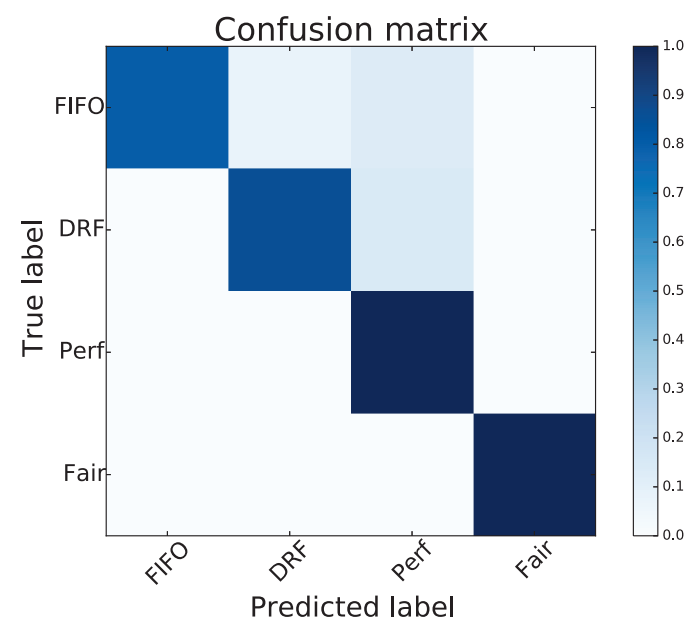

Figure 3.14: Confusion matrix (Facebook workload)

\begin{tabular}{|l|l|l|l|}
\hline & Precision & Recall & F1-score \\
\hline FIFO & $100 \%$ & $80 \%$ & $88 \%$ \\
\hline DRF & $92 \%$ & $86 \%$ & $89 \%$ \\
\hline Perf & $80 \%$ & $100 \%$ & $89 \%$ \\
\hline Fair & $100 \%$ & $100 \%$ & $100 \%$ \\
\hline avg & $93 \%$ & $91 \%$ & $91 \%$ \\
\hline
\end{tabular}

Figure 3.15: Classification accuracy of the decision tree (Facebook workload)

Cross validation with different workloads. In order to evaluate the robustness of the decision tree model across different workloads, we train our decision tree with Facebook workload and evaluate its accuracy with the Google workload. The confusion matrix is shown in Figure 3.16 and the related metrics are shown in Figure 3.17. The average precision, Recall and F1-score are all larger than $80 \%$ which further indicates that our model is accuracy even with different workloads.

Impact of the prediction error on resource efficiency. We study the impact of the prediction error of our workload model on the resource efficiency. Figure 3.18(a) shows the makespan reduction of FLEX compared with Tetris by introducing different prediction errors. The makespan reduction decreases slightly with the increase of the prediction error while our meta-scheduler still reduces the makespan significantly compared with Tetris. Specifically, the allowable threshold on the fairness 


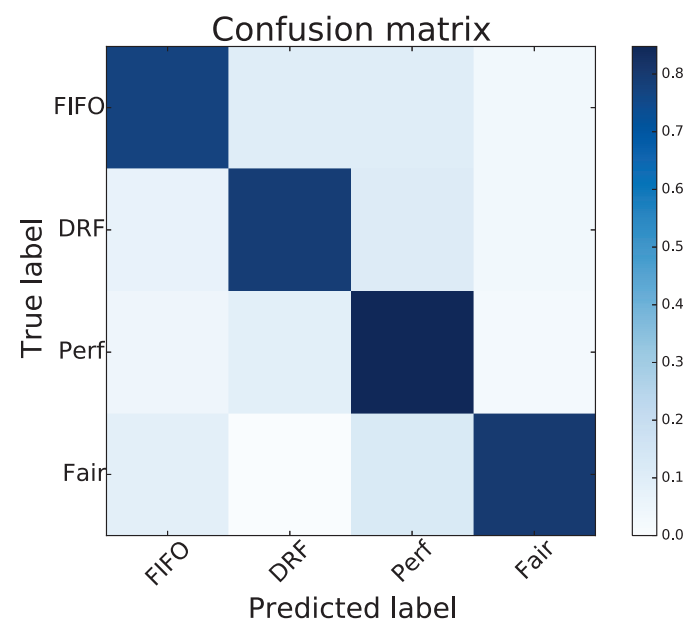

Figure 3.16: Confusion matrix (Different workloads)

\begin{tabular}{|l|l|l|l|}
\hline & Precision & Recall & F1-score \\
\hline FIFO & $80 \%$ & $77 \%$ & $78 \%$ \\
\hline DRF & $81 \%$ & $79 \%$ & $80 \%$ \\
\hline Perf & $72 \%$ & $85 \%$ & $78 \%$ \\
\hline Fair & $90 \%$ & $80 \%$ & $85 \%$ \\
\hline avg & $81 \%$ & $80 \%$ & $80 \%$ \\
\hline
\end{tabular}

Figure 3.17: Classification accuracy of the decision tree (Different workloads)

loss $w$ is $8 \%$ here. Given the a prediction error $e$, the prediction has a probability which is randomly distributed in $[w, w(1+e)]$ to randomly choose one scheduler from the candidate schedulers. We vary $e$ from $0 \%$ (no error) to $30 \%$. The result demonstrates the robustness of our optimizations, if the prediction error is reasonable.

Impact of prediction error on fairness. We also show the impact of prediction errors on the fairness loss reduction of Flex compared with Tetris in Figure 3.18(b). The fairness loss reduction slightly decreases with the increase of prediction error while our meta-scheduler still reduces the fairness loss significantly compared with Tetris. 


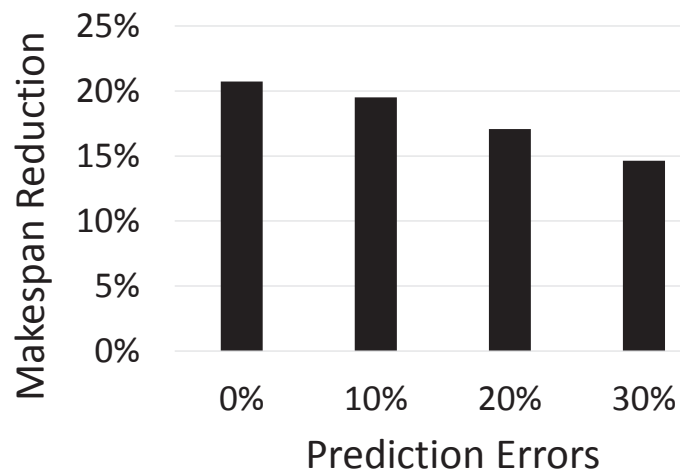

(a) The makespan reduction for different predic- (b) tion errors

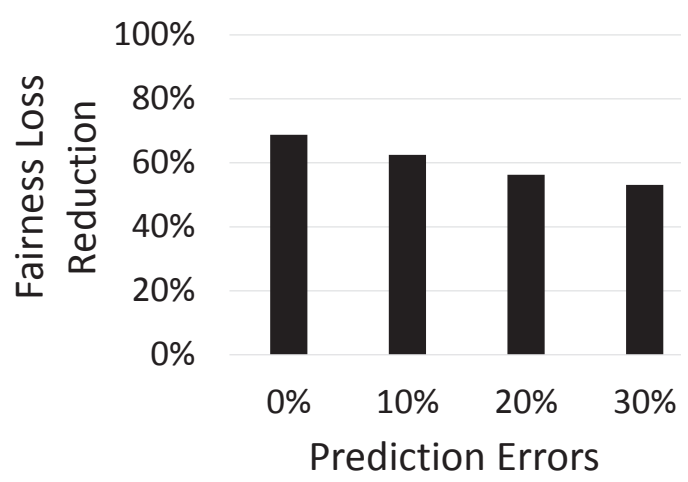

(b) The fairness loss reduction for different prediction errors

Figure 3.18: The reduction on the makespan and fairness loss of FLEX compared with Tetris for different prediction errors

\subsubsection{Impact of Long-term Fairness Definitions}

The fundamental difference between multiple fairness-oriented schedulers is that they have different efficiency-fairness tradeoffs. The choice of different fair schedulers depends on three major factors, including the workload characteristics, the user-defined SLA and the efficiency-fairness tradeoff of the fair schedulers. In fact, our meta-scheduler already integrates two fair schedulers into our system: Fair scheduler and DRF scheduler. Fair scheduler only considers memory. DRF is the state-of-the-art fair scheduler by considering multiple resource types and is treated as the baseline for comparison in our experiment. Figure 3.11(b) only utilizes the Fair scheduler in our meta-scheduler and Figure 3.11(c) only considers the DRF scheduler in the meta-scheduler. In Figure 3.11(b), Fair scheduler violates user-defined fairness loss requirement for some workloads. Therefore, the makespan reduction is penalized and the penalization decreases with the loose of the fairness constraint. In Figure 3.11(c), the makespan reductions for different thresholds on the fairness loss are all zero as DRF scheduler is treated as the comparison baseline. In Figure3.12(d), both Fair scheduler and DRF scheduler are integrated into our meta-scheduler and their cooperation actually achieves higher makespan reduction under the fairness constraint compared with the individual scheduler in Figure 3.11(b) and 
Figure 3.11(c). For some workloads, Fair scheduler is selected by the meta-scehduler because it is able to reduce makespan while satisfying the user-defined threshold on the fairness loss. For other workloads, DRF scheduler is chosen by the meta-scheduler to guarantee the user-defined threshold on the fairness loss.

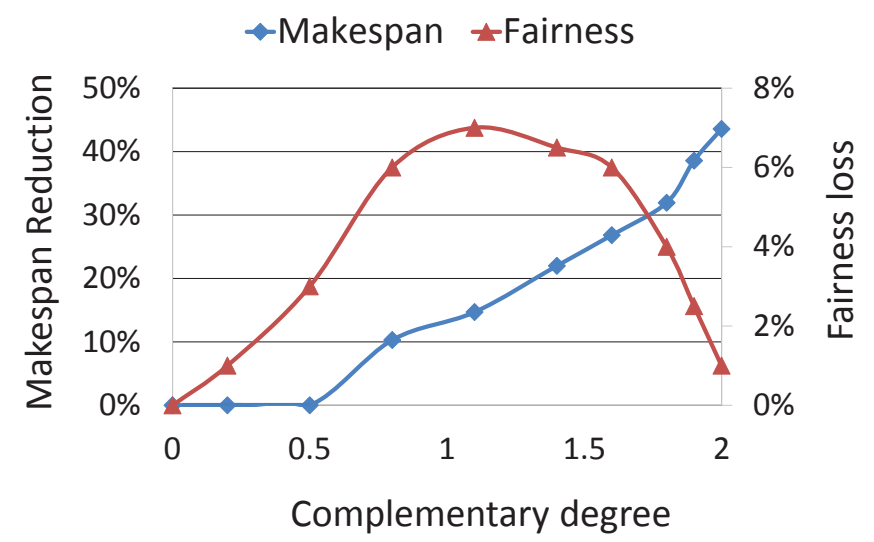

Figure 3.19: Tradeoff between the efficiency and fairness of efficiency-oriented scheduler for workloads with different complementary degrees. (long-term fairness)

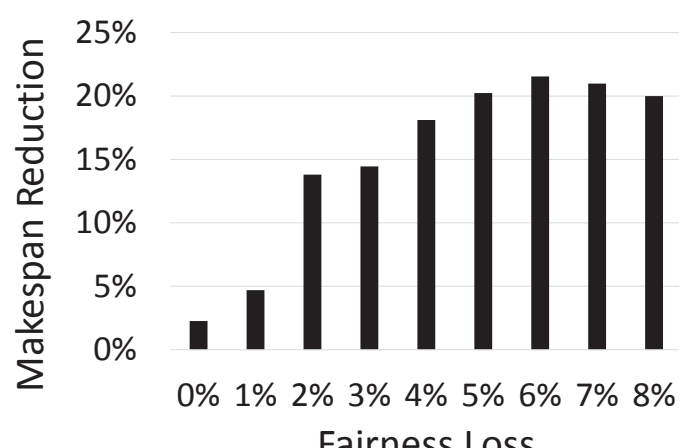

Fairness Loss

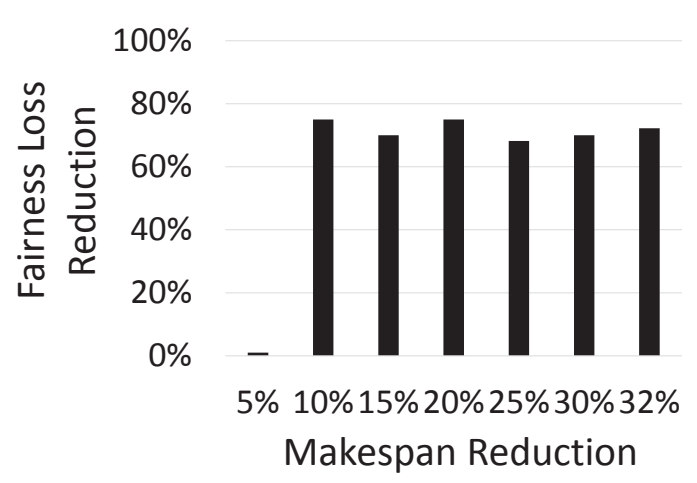

(a) Makespan reduction for different fair losses

(b) Fairness loss reduction for different makespan reductions

Figure 3.20: The reduction on the makespan and fairness loss of FLEX compared to Tetris (long-term fairness)

Both Fair and DRF only consider instantaneous resource allocation on a snapshot. In order to evaluate the fairness in one period, we apply the concept of long-term fairness [33] that ensures the fair allocation among multiple users along the time of their execution and the fairness is quantified 
from resource aspect by considering actual received allocation and purported fair allocation of all applications over their runtime. We make the following observations. First, there is still a tradeoff between the resource efficiency and this long-term fairness, which is shown in Figure 3.19. This tradeoff also depends on the workload that further verifies the importance of the adaptive scheduler in bi-criteria optimization for resource efficiency and fairness. Second, the fairness loss reduction under the long-term fairness definition is slightly higher compared with that under the definition in Section 3.4.2.1, due to the interference in the shared environment. In the context of this new fairness definition, FLEX still achieves significant makespan reduction compared to Tetris for the different thresholds on the fairness loss (as shown in Figure 3.20(a)). Similar, for the different thresholds on the makespan reduction, FLEX also significantly reduces the fairness loss, compared with Tetris (as shown in Figure 3.20(b)).

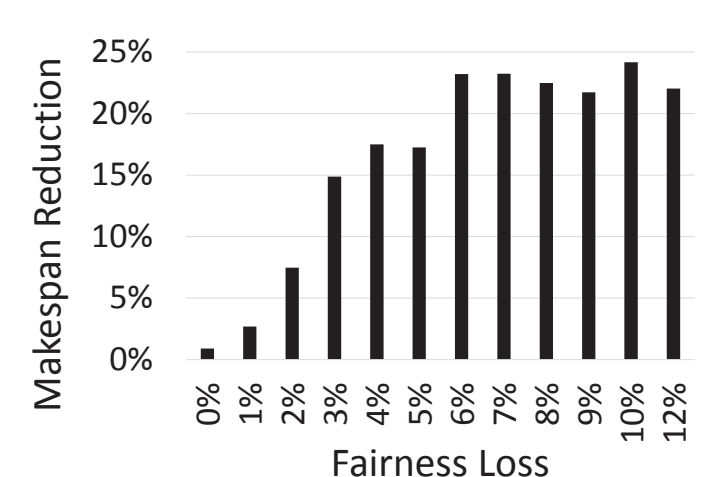

Fairness Loss

(a) Makespan reduction for different fair losses

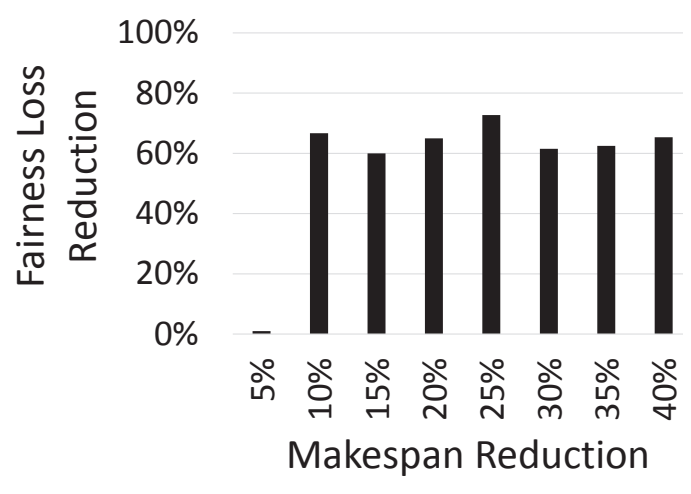

(b) fairness loss reduction for different makespan reductions

Figure 3.21: The reduction on the makespan and fairness loss of FLEX compared to Tetris in largescale simulation with Google trace

\subsubsection{Trace-driven Simulations}

Here, we evaluate the resource efficiency improvement and fairness loss of FLEX at a larger scale by mimic scheduling in a Google cluster using the production trace provided by Google. 
Figure 3.21(a) shows the makespan reduction of FLEX compared with Tetris for different thresholds on the fairness loss and Figure 3.21(b) gives the result of the reduction on the fairness loss of FLEX compared to Tetris for different thresholds on the makespan reduction. Similar to the results in our local cluster, FLEX can achieve better results than Tetris. We highlight with the following observations for the simulations with the production trace. For the same threshold on the fairness loss in Figure 3.21(a), the makespan reduction in the simulation is slightly higher than that of the local cluster, because our trace-driven simulator considers more resource types provided in Google

trace. Instead, our prototype implementation only considers two resource types as Hadoop YARN currently only supports the allocation of CPU and Memory. This results in more fragmentation and over-allocation of resources.

\subsection{Conclusion}

This study shows that due to the heterogeneous demand of multiple resources for users' jobs, being aware of the variation of the resource demand of the running workload is non-trivial for bi-criteria optimization between resource efficiency and fairness. There is a need to bridge this gap by studying the impact of workload's demand variation on the resource efficiency and fairness optimizations. In view of this, we propose a meta-scheduler called FLEX to realize the tradeoff between resource efficiency and fairness in Hadoop YARN. The experiments on real clusters and simulations show that FLEX achieves better resource efficiency as well as fairness than the state-of-the-art work. 


\section{Chapter 4}

\section{JouleMR: Towards Cost-Effective and Green-Aware Data Processing Frameworks}

In this chapter, we present our study for the cost-effective and green-aware schedulers for the dataintensive applications.

\subsection{Introduction}

Renewable energy and dynamic pricing are two major emerging trends in the energy management of modern datacenters. To leverage the renewable energy, many data centers have been built, being powered at least partially by renewable energy (or green energy, such as solar and wind). They are re-designed and integrated with the intelligence of smartly drawing power from multiple sources, including green energy from renewable and non-polluting sources and brown energy from traditional polluting sources [62]. Under this context, green-aware systems that integrate the awareness of renewable energy have become a hot research topic (e.g., [41, 57, 63, 90, 49, 65, 53]). In modern smart grids, the electricity price can be varying over time. Moreover, the variations of the price are substantial, as much as a factor of 10 between peak and non-peak periods [50]. Taking a special consideration on the dynamic pricing, cost-aware systems are proposed to exploit this variation for significant economic benefits $[50,51]$. 
Data processing frameworks (such as MapReduce [7]) are becoming important workloads in datacenters, which contribute to significant portions of energy consumption and electricity cost. Particularly, we consider each job can be delayed by a fixed amount of time and we have the opportunity to leverage green energy and the dynamic pricing by designing green-aware and cost-effective scheduling algorithms. Overall, this thesis investigates whether and how we can decrease the brown energy consumption and electricity cost for data processing frameworks in the data center with both brown and renewable energy supply.

Considering leveraging renewable energy and dynamic pricing schemes in data processing frameworks, we find that the key challenge is that they are both time-varying. Take renewable energy as an example. The renewable energy supplies are intermittent because of daily/seasonal effects and the prices of the electricity markets are varying on an hourly basis. Thus, the renewable energy supply or the low-price electricity may not match the workload demands, which results in severe under-utilization of renewable energy in a non-green-aware system or significant financial burden in a non-cost-effective system. Green-aware systems (e.g., [49, 65, 57]) and cost-effective systems (e.g., $[50,51])$ have been developed to address the mismatch in the context of data centers. The core ideas behind those studies are similar: they delay workloads according to jobs' deadline to match the renewable supply or the low-price electricity.

While those green-aware algorithms and systems can exploit the green energy and dynamic pricing at a coarse-grained level, they fail to capture the efficiency of each joule, which leads to severe waste of renewable energy and brown energy. We define the concept of joule efficiency to be the amount of work that can be done by a joule. Not all joules are equal in the sense that the joule efficiency of the energy can vary significantly, depending on when the energy comes and how the energy is used in computing. Ignoring joule efficiency can lead to significant energy waste (by $25 \%$ of the total energy consumption of Hadoop YARN on a Facebook production trace according to our study in Section 4.5). 
We have observed a number of key factors resulting in joule efficiency of data processing frameworks in data centers. First, data processing frameworks, particularly MapReduce, have non-linear performance-energy consumption speedups when the number of machines scales. Figure 4.1 shows the normalized performance and energy consumption for running three MapReduce jobs. All the three jobs are WebdataScan operations in the GridMix benchmark, with different selectivities in our local cluster. More details of the experimental setup can be found in Section 4.5. The major observation is that running on fewer nodes is actually more energy efficient (the total energy consumed for the job is lower) than running on more nodes because the network I/O becomes the bottleneck as the increase of the data to transfer. Therefore, one joule of energy can result in different amounts of work done, depending on the workload characteristics and the number of machines involved in the job. The largest difference is around 35\%, which shows the significant energy waste if the non-linear performance-energy consumption phenomenon is ignored. Second, battery has become an integral component for many data centers [91, 92, 93]. Charging/discharging causes inherent energy loss. Ideally, a green-aware and cost-effective system should be aware of not only the green energy supply and the dynamic pricing, but also joule efficiency to maximize the effectiveness of utilizing green energy as well as dynamic pricing.

In this thesis, we propose JouleMR, a cost-effective and green-aware MapReduce framework. With the slack time on each job, we develop job/task scheduling algorithms with special considerations on the factors on joule efficiency in the data center, including the energy efficiency of MapReduce workloads, renewable energy supply, dynamic pricing and the battery usage. Since finding the optimal scheduling solution is a NP-hard problem, JouleMR embraces a series of simple and effective heuristics for optimizations. We further develop a performance-energy consumption model. The model guides us to make the scheduling decision so that the deadline can be met and joule efficiency can be accurately estimated for scheduling the job/task at appropriate time.

We have implemented JouleMR on top of the latest Hadoop (Hadoop YARN 2.6.0). We evaluate JouleMR in two complementary approaches. First, we run real experiments with JouleMR on a local 


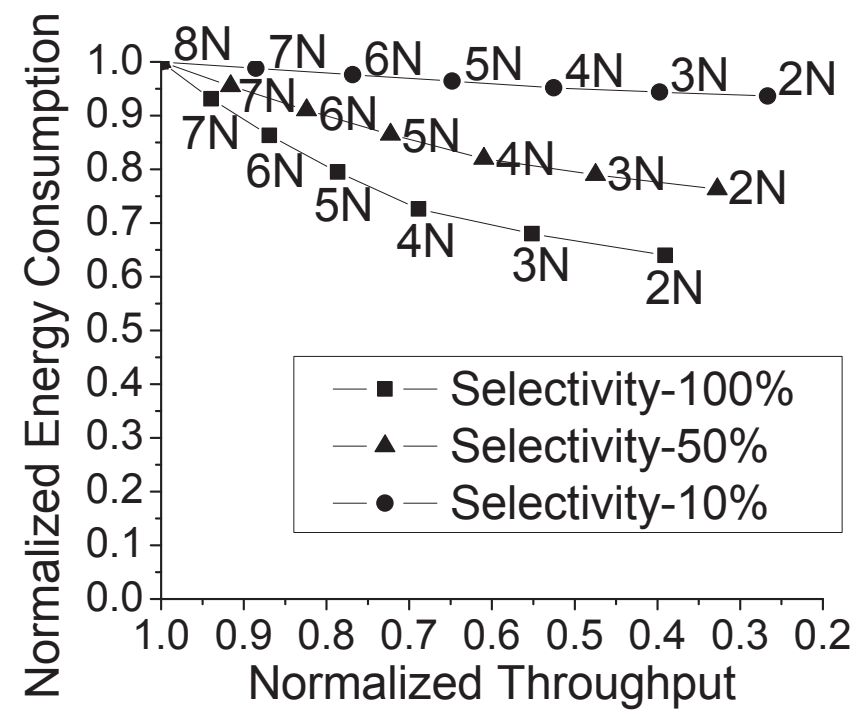

Figure 4.1: Energy consumption and performance tradeoff of Hadoop YARN in our local cluster. " $x \mathrm{~N}$ " means the result is obtained by running on $x$ nodes. Selectivity is defined as the ratio between the amount of output data of the map phase and the total input size of the benchmark (500GB in the compressed format).

cluster with micro benchmarks. Second, to allow more comprehensive studies, we have developed a simulator that replays the traces from the Facebook Hadoop cluster. Our experimental results demonstrate that 1) Our performance-energy consumption model can accurately predict the performance and energy consumption of our local cluster, 2) JouleMR significantly reduces the brown energy on both real experiments and simulations compared with GreenHadoop (the state-of-the-art green-aware Hadoop) [57] (up to $35 \%$ and $28 \%$ reduction, respectively). 3) JouleMR reduces the electricity cost on both real experiments and simulations compared to GreenHadoop (support dynamic pricing) (by $30 \%$ and $36 \%$ reduction, respectively).

\subsection{Problem Definition and Overall Design}

\subsubsection{Problem Definition}

In this thesis, we consider reducing the brown energy usage of data processing frameworks (particularly MapReduce) in a single data center. The data center is powered by both brown energy and green 


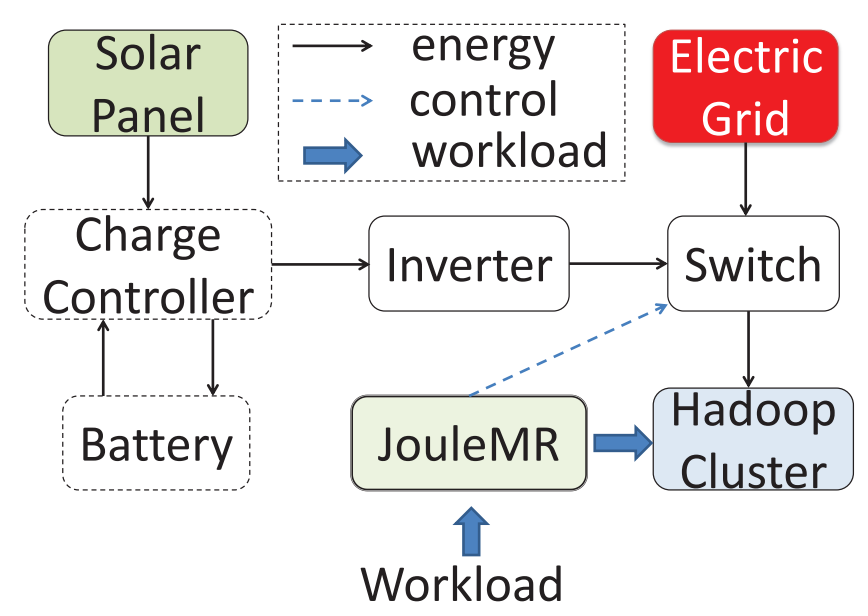

Figure 4.2: System overview of JouleMR

energy sources, with battery supports.

User constraints like deadlines are important requirements for the performance of data processing environments such as Hadoop. Thus, JouleMR allows users to specify the slack of a MapReduce job: the slack of each job is defined according to its specified deadline. JouleMR can leverage the slacks of these jobs to perform green-aware and cost-effective optimizations. We note that the deadline is "soft", mainly representing the quality of service.

The system overview of JouleMR is shown in Figure 4.2. The system consists of a Hadoop cluster, a charge controller, an inverter, batteries and a switch. Because the voltage of the green energy sources changes over time, we use charge controller to prevent any overcharging. The charge controller also monitors the charging/discharging operations. The surplus green energy is charged into battery automatically.

Effort has been devoted to optimize the smart switch connected with both brown and green sources sources [94]. When the supply of green power and battery is slower than the power demand, it immediately draws energy from brown energy sources.

Data centers may have centralized batteries in order to provide an uninterrupted power supply (UPS) for power failures. More recently, data centers also incorporate batteries at the rack- or even 
server-level, with higher energy efficiency than centralized battery. Previous studies [95] have demonstrated that batteries can be used to reduce the energy consumption cost in the scale of data centers. In order to leverage the batteries as an energy source to reduce the energy consumption as well as cost without significantly hurting their lifetime, similar to GreenSwitch [63], we limit the discharge depth to a fixed fraction of the maximum capacity at all times. The cost of the batteries is amortized during their lifetime. In our study, we estimate this component as the amortized cost multiplied by the scheduling time. Without loss of generality, we express the battery model with two parameters $<P, e>$, in which $P$ is the effective capacity of the battery that can be used for discharging and $e$ is the charging efficiency of the battery $(0 \leq e \leq 1)$. For a given charging power $\Delta x$, we calculate the increment of energy storage as $\Delta x^{\prime}=\Delta x \cdot e$. Here, we assume the charging efficiency as constant for simplicity. JouleMR can be extended to handle other battery models with variable efficiency. Due to the inherent charging/discharging loss, the battery is either charged or discharged at each time.

Optimization goal. In this section, we give the formulation of our optimization problem. We assume that the workload consists of $N$ jobs. Each job has a user-defined deadline. We have a greenaware optimization goal and a cost-effective optimization goal which aim at the two major trends in energy management. Given the MapReduce workload with bounded slacks, JouleMR schedules the workloads based on the joule efficiency and the green energy supply dynamically. Our green-aware optimization goal is to minimize the total brown energy consumption, under the constraints that all workloads are completed before their predefined deadlines. Besides, we extend our system to leverage the dynamic pricing. The cost-effective optimization goal is to minimize the electricity cost, given the jobs' deadline constraint. The brown energy consumption at time slot $i$ is $b_{i}$ and the brown energy price at time slot $i$ is $p_{i}$. The total amount of brown energy $B$ is easily calculated as

$$
B=\sum_{i \in(0, T)} b_{i}
$$


, where $T$ is the finishing time of all jobs and the green-aware optimization goal is to minimize $B$. The total monetary $\operatorname{cost} C$ is calculated as

$$
C=\sum_{i \in(0, T)} b_{i} * p_{i}
$$

The green-aware optimization goal is to minimize $C$. We then give the constraints of our optimization problem. The constraints consist of job-related constraints and energy-related constraints.

Job-related constraints. We first describe the job-related constraints. All MapReduce jobs need to finish before their deadlines. We define the the deadline of job $i$ as $d_{i}$ and finishing time of job $i$ as $f_{i}$. We can easily get

$$
\forall i, f_{i} \leq d_{i}
$$

A MapReduce job consists of map tasks and a reduce tasks. If the finishing time of all map tasks of job $i$ is $m_{i}$ and the start time of reduce phase of job $i$ is $r_{i}$. The reduce task can not start until all map tasks finishe. Thus, we have

$$
\forall i, m_{i} \leq r_{i} \leq f_{i}
$$

Then, we calculate the execution speed of each job. We use $W M_{i j k}$ to indicate whether the machine $i$ is allocated to map tasks of job $j$ at time slot $k$ or not. If $W M_{i j k}$ is equal to 1 , then it means that the machine $i$ is allocated to map tasks of job $j$ at time slot $k$. Otherwise, $W M_{i j k}$ is equal to 0. Similarly, we use $W R_{i j k}$ to indicate whether the machine $i$ is allocated to reduce tasks of job $j$ at time slot $k$ or not. We assume each machine can be allocated to map tasks or reduce tasks of one job at one time.

$$
\sum_{j}\left(W M_{i j k}+W R_{i j k}\right) \leq 1, \forall i, k
$$

We now calculate the execution speed of map tasks and reduce tasks, respectively. First, we calculate the execution speed of map tasks. Each map task reads its input off the local disk, executes 
the map function and writes the intermediate data to local disk. Thus, a map task involves the disk and CPU, and its execution speed is decided by the slowest of the two resources. We define the execution speed of one map task of job $j$ on machine $i$ as $S M_{i j}$. Hence,

$$
S M_{i j}=\min \left\{B D_{i}, B M_{j}\right\}
$$

, where $B D_{i}$ is the disk IO bandwidth per task on machine $i$ and $B M_{j}$ is the processing rate of map function of job $j$. If the CPU demand and memory demand of one map task of job $j$ are $C M_{j}$ and $M M_{j}$, respectively. Then, the number of concurrent executing map tasks of job $j$ on machine $i$ is calculated as

$$
N M_{i j}=\min \left\{\frac{C_{i}}{C M_{j}}, \frac{M_{i}}{M M_{j}}\right\}
$$

, where $C_{i}$ is the CPU cores of machine $i$ and $M_{i}$ is the memory capacity of machine $i$. We finish all map tasks of job $j$ before time $m_{j}$. Hence,

$$
\forall j, \sum_{k=0}^{m_{j}} \sum_{i=0}^{M}\left(\frac{S M_{i j}}{I_{j}} * N M_{i j} * W M_{i j k} * T\right) \geq U_{j}
$$

, where $M$ is the total number of machines, $I_{j}$ is input size of one map task of job $j, T$ is the time slot length and $U_{j}$ is the total number of map tasks of job $j$. Second, we calculate the execution speed of reduce tasks. Each reduce task consists of two phases, shuffle phase and computing phase. In shuffle phase, the input data is read remotely from the network, merged and written to the local disk. Next, the data is read off the disk and an external merge sort is performed before the data is consumed by the reduce function. The merge sort may need multiple steps if the data size is too large. We uses a factor $f$ to capture the extra data written. Thus we calculate the execution speed of reduce task of job $j$ on machine $i$ as

$$
S R_{i j}=\min \left\{\frac{B D_{i}}{f}, B N_{i}, B R_{j}\right\}
$$


, where $B D_{i}$ is disk bandwidth per task on machine $i, B N_{i}$ is the network bandwidth per task on machine $i$ and $B R_{j}$ is the processing rate of reduce function of job $j$. If the CPU demand and memory demand of one reduce task of job $j$ are $C R_{j}$ and $M R_{j}$, respectively. Then, the number of concurrent executing reduce tasks of job $j$ on machine $i$ is calculated as

$$
N R_{i j}=\min \left\{\frac{C_{i}}{C R_{j}}, \frac{M_{i}}{M R_{j}}\right\}
$$

Similarly, we finish all reduce tasks of job $j$ before time $f_{j}$. Hence,

$$
\forall j, \sum_{k=r_{j}}^{f_{j}} \sum_{i=0}^{M}\left(\frac{S R_{i j}}{I_{j} * S_{j}} * N R_{i j} * W R_{i j k} * T\right) \geq V_{j}
$$

, where $S_{j}$ is selection ratio of map tasks of job $j$ and $V_{j}$ is the total number of reduce tasks of job $j$.

Energy-related constraints. We define the brown energy usage at time slot $i$, the amount of green energy supply at time slot $i$, the total energy consumption of the cluster at time slot $i$ and the amount of energy charged/discharged into/from the battery at time slot $i$ as $B_{i}, G_{i}, E_{i}$ and $P_{i}$, respectively. $B_{i}$, $G_{i}$ and $E_{i}$ are all larger than or equal to 0 . If $P_{i}$ is larger than 0 , it means that energy is charged into the battery. Otherwise, it means that the energy is discharged from the battery. For each time slot $i$, the total amount of the energy consumed by the cluster and the amount of energy charged into the battery is less than or equal to the sum of the amount of brown energy drawn from the electric grid and the amount of green energy from the solar panels, which is shown as

$$
B_{i}+G_{i}>=E_{i}+P_{i}
$$

$E_{i}$ is the total amount of energy consumption of all machines at time slot $i$.

$$
E_{i}=\sum_{j} E M_{j i}
$$

, where $E M_{j i}$ is the energy consumption of machine $j$ at time slot $i$. The energy consumption of one machine is decided by the cpu utilization according to our cost model which is described in detail in 
section 4.4. Hence, we calculate the energy consumption of machine $j$ at time slot $i$ as

$$
E M_{j i}=\sum_{k}\left(W M_{j k i} * f_{j}\left(U M_{k j}\right)\right) * T+\sum_{k}\left(W R_{j k i} * f_{j}\left(U R_{k j}\right)\right) * T
$$

, where $f_{j}$ is the energy cost function of machine $j, U M_{k j}$ is the cpu utilization of map tasks of job $k$ on machine $j$ and $U R_{k j}$ is the cpu utilization of reduce tasks of job $k$ on machine $j . U M_{k j}$ and $U R_{k j}$ can be easily calucated as

$$
\begin{aligned}
U M_{k j} & =\frac{S M_{k j}}{B M_{k}} \\
U R_{k j} & =\frac{S R_{k j}}{B R_{k}}
\end{aligned}
$$

In each time slot, the energy can be charged into or discharged from the battery. The energy discharged from the battery is constrained by the amount of available energy of battery and the energy charged into the battery is also constrained by the capacity of the battery. Hence,

$$
0 \leq A_{i}+P_{i} \leq P
$$

, where $A_{i}$ is the amount of available energy in the battery at time slot and $P$ is the effective battery capacity.

In summary, we formulate our optimization problem as a temporal knapsack problem [101] with job-related and energy-related constraints.

\subsubsection{System Overview}

Supply driven execution (SDE) is a common approach which is utilized in green-aware and costeffective systems in the data center with multiple energy sources [41, 57, 90, 49, 65, 53]. By delaying the workload, the mismatch between the green supply (or low-price brown energy) and the workload demand can be resolved. However, SDE treats each joule of energy the same without considering the phenomenon of joule efficiency caused by workload scheduling. Thus, SDE can severely waste energy due to its obliviousness to joule efficiency. 


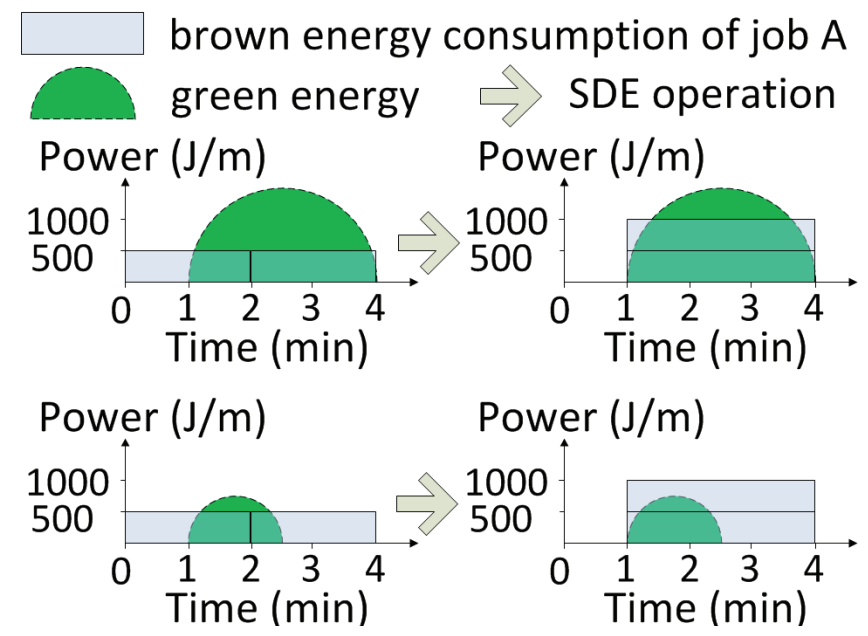

Figure 4.3: Two illustrative examples: (Top) SDE reduces the brown energy consumption; (bottom) SDE increases the brown energy consumption.

Let us study two simple examples shown in Figure 4.3. If we execute job A on one machine, its execution time is 4 minutes and energy consumption is $2000 \mathrm{~J}$. On the other hand, if we execute it on two machines, its execution time is 3 minutes and energy consumption is $3000 \mathrm{~J}$. Due to the nonlinear speedup feature of job A, increasing the computing resources by a factor of two allows us to delay the execution, but does not reduce the execution time by half. Thus, more energy is consumed if we increase the number of machines. SDE cannot fit all for different green distributions. In the above case of Figure 4.3, SDE tends to consume less brown energy. On the contrary, in the case below, executing the job earlier can actually consume less brown energy. This example shows SDE fails to capture joule efficiency on non-linear performance and energy consumption of data processing frameworks. We can easily give some examples from other joule efficiency (e.g., battery) to show that SDE does not always reduce the brown energy consumption.

There are three main technical issues for JouleMR. First, SDE defers too much workload which may cause excessive deadline violations because of the limited capacity of the Hadoop cluster. Second, we should have a systematic way of scheduling MapReduce jobs by considering joule efficiency: when to schedule the job and how many resources should be allocated to the job in order to utilize 
the brown as well as green energy efficiently. Third, integrating battery into system can optimize the intermittent green energy usage and the pricing scheme, with the overhead in charging/discharging.

We propose a MapReduce framework aware of joule efficiency (JouleMR), and develop effective cost models and efficient optimization algorithms to address the above-mentioned technical issues. The overall design of JouleMR is described in Algorithm 2. When a new job comes to the system, it is initially put into a job waiting queue. For the jobs in the job waiting queue, we need to conduct the performance and energy consumption estimations on the job (Line 2). With these estimations, we determine the most energy-efficient execution configuration that satisfies the deadline (Line 3). The configuration includes the number of servers and the amount of physical resources per server. Then, we adds the job to the job queue (Line 4). In our implementation, we use a multi-queue structure to express the energy-efficient execution plan of the workload.

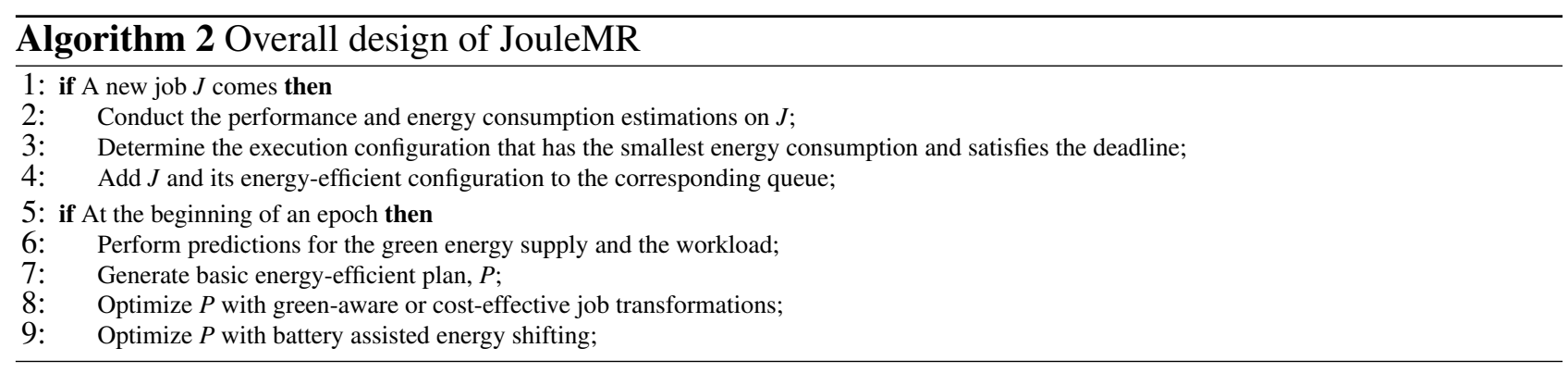

As MapReduce jobs are coming to the system in an ad hoc manner and the green energy is varying over time, it is hardly feasible to know all future information at one moment and make an optimal offline scheduling plan. The scheduling plan should be generated and adjusted at runtime to adapt to the variance of the workload and green energy. In this study, we perform the adjustment periodically for simplicity of algorithm design. JouleMR periodically generates the energy-efficient and green-aware (or cost-effective) scheduling plan (Lines 5-9) (the period is called epoch). We perform predictions on the green supply and the workload at the beginning of each epoch. Given the energy-efficient execution configuration of each job, we first generate the basic energy-efficient plan according to 
the cluster capacity. Next, we apply a series of optimizations to reduce the brown energy consumption (or electricity cost) of the plan, including green-aware or cost-effective job transformations and battery assisted energy shifting. The generation of the basic energy-efficient plan, green-aware or cost-effective job transformations and battery assisted energy shifting are orthogonal with each other, and we will evaluate their separate impact in Section 4.5.

JouleMR periodically schedules jobs/tasks and performs power management on servers according the optimized execution plan (the period is called slot). In our design, we consider fine-grained jobs/tasks scheduling and power management and an epoch consists of multiple slots. For each time slot, JouleMR decides the set of active servers and allocates them to jobs and executes them according to the execution plan. For all jobs to be scheduled in the execution plan of current slot, JouleMR adopts the deadline first scheduling algorithm, which gives priority to the jobs that are close to deadlines.

Finally, we note that the epoch/slot design improves the robustness of JouleMR to inaccurate energy usage predictions or other dynamic factors including hardware failure, I/O contention and workload imbalance [96]. JouleMR reacts to and re-evaluates the system state for each epoch/slot. For example, if the current epoch is over-estimating the energy consumption, it may utilize more brown energy when not necessary. However, these scheduled jobs can finish faster than expected, which causes JouleMR to adjust by scheduling more jobs from the job queue. In the next sections, we describe the details on the green-aware and cost-effective scheduling of JouleMR in Section 4.3 and Section 4.4, respectively.

\subsection{Green-aware Scheduling}

In this section, we describe how to minimize the brown energy consumption of the cluster through green-aware scheduling. We first generate a basic execution plan with special considerations on energy efficiency of data processing frameworks and cluster capacity. Next, we further reduce the 
brown energy consumption of the basic energy-efficient plan by green-aware job transformations and battery assisted green shifting mechanisms.

\subsubsection{Basic Energy-efficient Plan}

At the beginning of each epoch, we perform online predictions for the supply of green energy and the workload (including job types and arrival intervals, etc.) for this epoch.

Workload Prediction. Generally, it is difficult to have an accurate prediction on the workload, because of the diversifying job sizes [97]. Fortunately, we observe that small jobs and large jobs in real production traces have very different arrival patterns (see the experimental setup in Section 4.5.1). Particularly, the arrival rate of small jobs (\# maps is less than ten in our experiment) can be predicted with relatively high accuracy by using some simple time series analysis [98, 99] (prediction error is less than $8 \%$ in average for the real production trace from facebook). In our experiment, we apply exponentially weighted moving average algorithm based on the historical statistics data to predict the arrival rate for small jobs. The total energy consumption and execution time of these small jobs can be estimated accurately with similar time series prediction algorithms. In our experiments, the prediction error of energy consumption is less than $6 \%$ in average and prediction error of execution time is less than $4 \%$ in average.

As for large jobs, they come in a more ad hoc manner, and the energy consumption and execution time of large jobs vary significantly. Instead, we estimate large jobs individually using our cost model after they come. Since large jobs tend to have longer execution time, such an on-demand prediction has given sufficient optimization room for scheduling, as we observed in our experiments.

Green Supply Prediction. Green energy sources such as wind and solar are easily impacted by weather. A lot of existing weather learning methods and models are proposed (e.g., [57, 100]). This is not the focus of this thesis. We simply adopt the previous approach used in GreenHadoop [57], which has been shown to achieve very good accuracy for solar energy. Additionally, we also study the impact of prediction errors in the experiments. 
Execution Plan Generation. We now generate the basic energy-efficient plan for JouleMR. We note that, different from the SDE plan generated in GreenHadoop [57], the plan generated by JouleMR has two distinct features. First, our job execution configuration has the smallest energy consumption while satisfying the deadline. Additionally, the plan generation is guided by our energy consumption and performance model for MapReduce jobs, rather than heuristics [57].

We use a multi-queue data structure to represent the execution plan of an epoch. Each queue corresponds to one time slot and it contains the jobs/tasks starts at the beginning of the time slot. Each epoch contains $N$ time slots and duration of the slot is $t_{s}$ seconds, where one time slot corresponds to one queue. There are $N$ queues for the current epoch in our design, expressed with $q_{0}, q_{1}, \ldots, q_{N-1}$, where $q_{i}(0 \leq i<N)$ maintains all jobs with the slack time less than $(i+1) \cdot t_{s}$. A separate queue $q^{\prime}$ is used to store all jobs with the slacks beyond the current epoch. Suppose a job with a slack of $k$ is submitted after $t$ seconds of the beginning of current epoch, it will be added to $q_{\frac{t+k}{t_{s}}}$ if time $t+k$ is within current epoch, otherwise it is added to waiting queue $q^{\prime}$. As the time goes by, at the beginning of each time slot, the slacks of jobs belongs to the current queue will decreases to $\left[0, t_{s}\right]$, and they all will be assigned with the decided number of servers which are scheduled to run until all tasks completes. At the beginning of each epoch, jobs with the slacks smaller than the end of the epoch are distributed to corresponding $q_{i}$, and jobs have not finished in the previous epochs will be distributed to $q_{0}$. Thus, the jobs for an epoch include the workload from $q^{\prime}$, the jobs arriving inner the current epoch with emerging slacks which have to finish before the end of the current epoch and the jobs from the previous epochs.

After initiating each job into the corresponding queue, we ensure each slot can schedule all jobs/tasks allocated to it subject to the cluster capacity. Suppose the numbers of servers that are required in each time slot are $n_{i}(0 \leq i<N)$. If $n_{i}$ exceeds the cluster capacity, we need to move the jobs running in current time slot with the earliest submission time to the queue $j(j<i)$ with available resources. We repeat this process until all $n_{i}$ are no larger than the cluster's capacity. Otherwise, the cluster is overloaded. 


\subsubsection{Green-aware Optimizations with Job Transformations}

The MapReduce jobs consist of individually tasks which can be executed concurrently. Even the job has started, for all pending tasks, we can still dynamically adjust the number of concurrent running tasks at one moment by controlling the amount of resources allocated to the job as adjustment to the execution plan by job transformations. As observed in Figure 4.3, we can transform a job in a certain way so that it can decrease the usage of brown energy. We first define four basic job transformation operations, and next present the optimization techniques that leverage the job transformations.

JouleMR supports four basic job transformations:

1) Delay $(j, i)$ : it delays the starting time of job $j$ for $i$ time slots; 2$)$ Advance $(j, i)$ : it advances the starting time of job $j$ for $i$ time slots; 3$)$ Promote $(j, i)$ : it increases the number of machines allocated to the job $j$ by one in time slot $i$, so that it can complete faster but usually at the cost of more energy consumption; and 4) Demote $(j, i)$ : it reduces the number of machines allocated to the job $j$ by one in time slot $i$, which is a dual operation of promotion. Promotions and demotions capture the nonlinear performance and energy consumption in MapReduce jobs. Our cost model can predict the performance and energy consumption changes for the job transformations.

Those four operations can be used together to form a sequence of transformation operations. Additionally, the same operations can be used multiple times. For example, we should use promotions for $x$ times if we want to add $x$ machines to the job.

Given the generated basic energy-efficient plan of the current epoch and the predicted green energy distribution in each time slot, we need to perform the transformations for each job using the above four operations, and our goal is minimizing the total brown energy usage in the current epoch among all jobs. We need to decide how many machines to be assigned in each time slot for every job under the deadline and resource capacity constraints. We formulate this problem into a temporal knapsack problem [101] which is described in Section 4.2.1. Since finding the optimal solution to that problem is NP-hard, we propose a series of simple and effective heuristics for optimizations. Particularly, we 
propose a novel two-phase heuristics-based approach to solve this non-trivial optimization problem. Those two phases are complementary with each other. Phase 1 algorithm utilizes the delay and advance operations to shift the basic energy-efficient plan as a whole without impact on its energy efficiency. Phase 2 algorithm leverages the demote and promote operations to change the shape of the basic energy-efficient plan at the cost of the energy efficiency.

Phase 1: In this phase, we perform advance and delay operations to reduce the brown energy consumption while preserving the joule efficiency of the basic energy-efficient execution plan of the current epoch. As all jobs in the basic energy-efficient execution plan are delayed as late as possible, any delay operations will cause deadline violations. Therefore, we first perform the advance operations and then consider the delay operations if the brown energy consumption can be further optimized.

The optimization algorithm for Phase 1 is described in Algorithm 3. First, we perform advance operations on the basic energy-efficient execution plan. We iterate the execution plan in ascending order (from the first time slot to the last time slot in the epoch). For each time slot, we consider all jobs that are submitted before that time slot while start after that. If the brown energy consumption of the current epoch can be reduced by advancing the starting time of any job, we perform the advance operation on that job. Note, the brown energy consumption of an epoch is estimated to be $\sum_{i=1}^{N} \operatorname{Max}\left\{J_{i}-G_{i}, 0\right\}$, where $N$ is the number of time slots in one epoch, $J_{i}$ is the total energy consumption of all jobs running in time slot $i$ (estimated using the cost model), and $G_{i}$ is the predicted amount of green energy in time slot $i$. Then, we perform delay operations on the basic energy-efficient execution plan. We iterate the execution plan in reverse order (from the last slot forwarding to the first slot in the epoch). For each time slot, we consider all jobs start before that time slot. We iterate these jobs and perform delay operations on them if the brown energy consumption can be reduced. The time complexity of the phase 1 algorithm is decided by the number of slots in one epoch and the number of jobs. Suppose the number of time slots in one epoch is $N$ and the number of jobs is $M$. 
The time complexity of the phase 1 algorithm is $O(M N) . N$ is usually very small (one epoch consists of 20 time slots in our experiment).

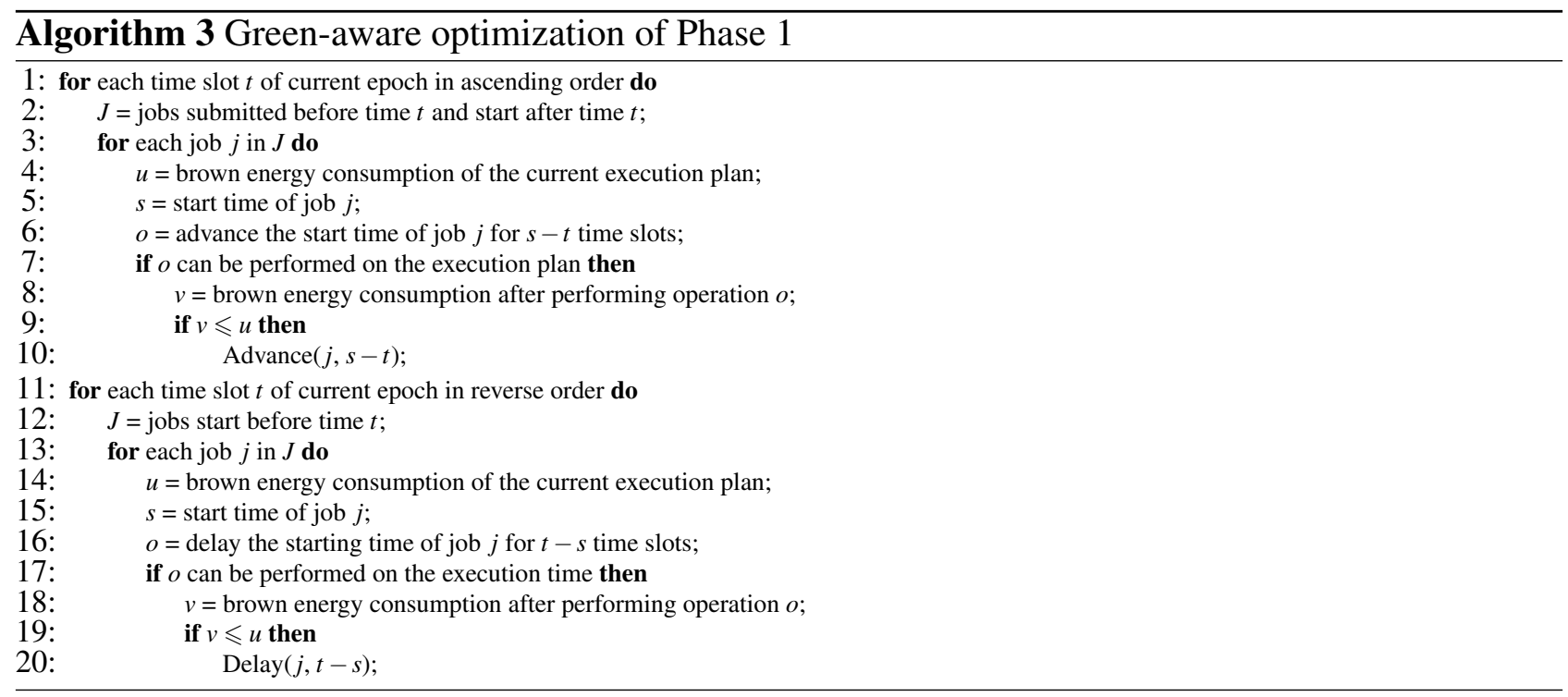

Phase 2: Complementary to Phase 1, we consider promote and demote operations on the jobs if those transformations can further reduce the brown energy consumption. Instead shifting an execution plan of a job as a whole, Phase 2 algorithm can change the shape of the execution plan at the cost of energy efficiency.

The optimization algorithm for Phase 2 is described in Algorithm 4. We iterate the execution plan optimized after Phase 1 in ascending order. For each time slot $t$ in the current epoch, we consider workload shifting through promote and demote operations to further reduce the brown energy consumption. We add all jobs, which will be scheduled in the current time slot, into a FIFO queue. We dequeue a job from the FIFO queue each time and relocate the machine assigned to the job in the current time slot to the slot with the most surplus green energy supply if the brown energy consumption can be reduced. The tasks to be scheduled on this machine are also shifted accordingly. After the shifting, we enqueue the current job in to the FIFO queue and repeats this process until the FIFO queue becomes empty. We choose the FIFO queue structure to make all jobs benefit from the green 
energy evenly, which also minimizes the total reduction of joule efficiency caused by the non-linear speedup feature of Hadoop. The time complexity of the phase 2 algorithm is $O\left(M N^{2}\right)$.

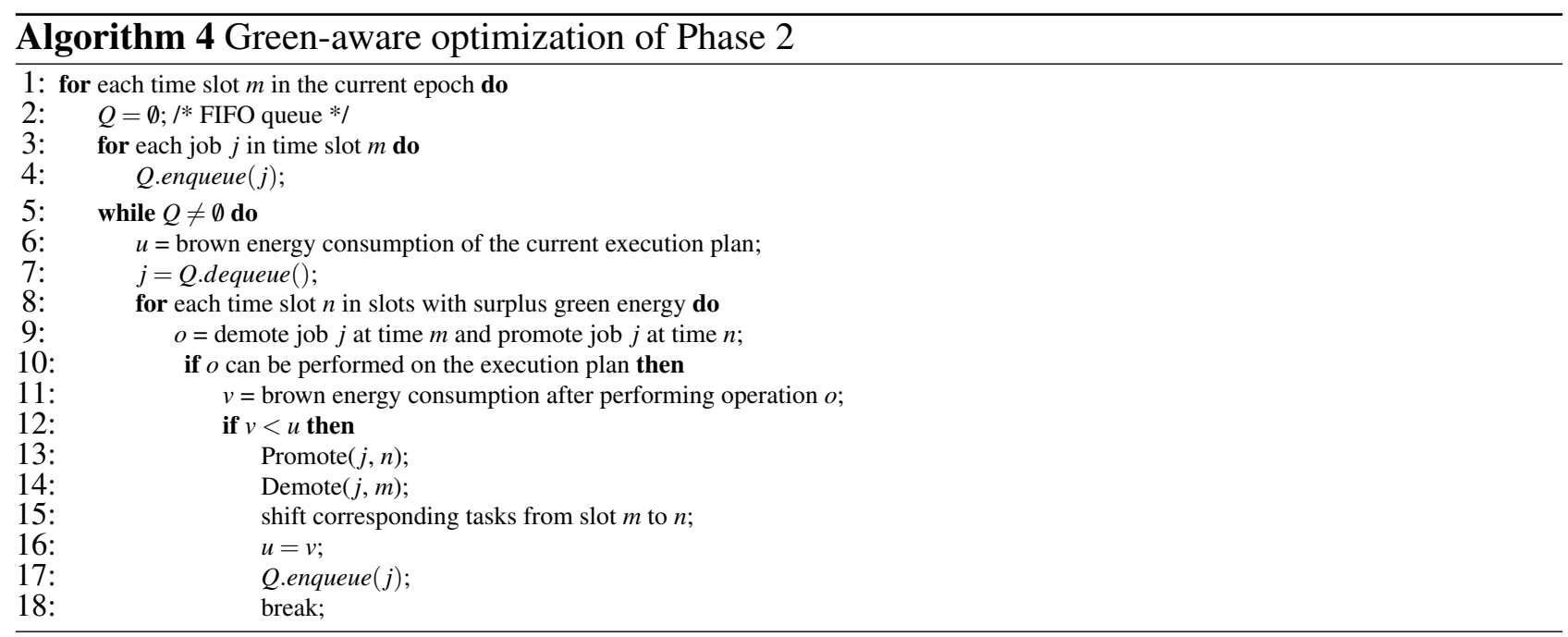

\subsubsection{Battery Assisted Green Shifting}

We can take advantage of battery to store the green energy for future use when the green energy supply is surplus. The basic approach adopted by the previous studies (e.g., [49, 63]) are as follows. The battery is charged only when there is surplus green energy (i.e., the green energy supply is higher than the total energy consumption). When the green supply is lower than the energy demand, the battery is first discharged. The discharging stops when the battery capacity reaches a predefined threshold, only for emergency usage, and then the brown energy is used.

However, the basic approach does not consider joule efficiency. If we find that storing the green energy can do more work in the future (green shifting) rather than using it at present, we can proactively store it into the battery despite of the charging loss. This charging method can be feasible, since MapReduce jobs exhibit non-linear speedup and energy consumption behavior.

With the consideration of joule efficiency, the charging of battery needs careful designs. One example is illustrated in Figure 4.4. In Figure 4.4 (a), all green energy is utilized directly. Instead, 


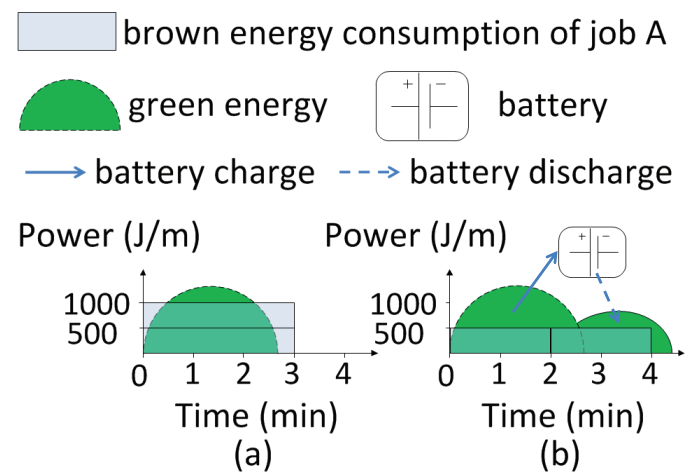

Figure 4.4: An example of battery assisted green shifting

Figure 4.4 (b), only partial green energy is used after a job transformation (demotion) and remainder is charged to the battery for future use. It results in significant brown reduction as energy is utilized more efficiently.

We propose our battery assisted green shifting as a further optimization on the execution plan optimized through job transformations. Our optimization is performed periodically at the beginning of each time slot. With the battery, we can delay workload to later slots for higher energy efficiency. As there is energy loss in charging/discharging, we need to assess the tradeoff between energy gain of battery assisted green shifting and the charging/discharging loss. Still, we can formulate this problem as a nonlinear integer programming problem with constraints (e.g., battery capacity and deadlines), which is NP-hard. Therefore, we propose a heuristic algorithm to perform the green shifting optimization on the execution plan after job transformations.

Green Shifting Algorithm: We iterate the execution plan in the ascending order. For each time slot, we consider performing demote and charging surplus green energy for future usage for each job in the current time slot. Similar to Algorithm 3, we firstly add all jobs $J$ powered by green energy into a FIFO queue $Q$, and then dequeue a job from $Q$ each time and perform demote as well as charging if brown consumption can be reduced. This process repeats until $Q$ becomes empty. For each job $j$ dequeued from $Q$, if demote will not cause deadline violation, we perform demote on job $j$. Demote operation on $j$ causes less workload to be scheduled as surplus green is not fully utilized, 
The surplus green energy can be charged to battery and used to schedule the reduced workload for higher energy efficiency in the later time slots. However, not all of the surplus green energy can be utilized in the future because of energy loss in charging/discharging and energy waste when battery is at full capacity. Therefore, we need to compare the energy consumption of the reduced workload scheduled in the future for higher energy efficiency with the actual amount of green energy calculated by subtracting energy loss and waste from the amount of surplus green energy. If the actual amount of green energy is more than the energy consumption of reduced workload to be scheduled in the future, we commit the demote operation as well as charging surplus green for future usage, and enqueue $j$ into $Q$ for further optimization. Otherwise, we withdraw the demote operation.

\subsubsection{Cost Model}

Since we use the performance-energy consumption models at run time, we aim at developing a lightweight yet sufficiently accurate cost model. Since small jobs (i.e., with less than 10 map tasks) are predicted with time series algorithms, the model applies to large jobs only.

We have a number of design considerations. First, we need to integrate the hardware profile including servers and network switches to quantitatively reflect the relationship between utilization and energy consumption. We assume that the cooling energy consumption is strongly correlated to the total energy consumption of servers and network switches, and exclude the cooling energy consumption in our estimation for simplicity. Second, we need to have the total execution time and energy consumption of a MapReduce job, for the effectiveness of any scheduling decision. Third, the runtime overhead of the cost model should be low. There are a number of performance models for MapReduce (e.g., [102, 103, 104]). We extend the prediction model used in Bazaar [102] according to our design principles for guiding the scheduling decisions of JouleMR. There is a tradeoff between runtime overhead and model accuracy. Our design decision leans towards simplicity with sufficient accuracy. 
Overall, our performance-energy consumption model estimates the energy consumption and execution time, given the program for a MapReduce job and the execution configuration as input.

Table 4.1 lists the parameters used in the cost model. The user of JouleMR does not need to know the details of the performance-energy consumption model. With the integration into YARN, our cost model mainly relies on three kinds of parameters: MapReduce execution parameters, hardwarerelated parameters and intermediate as well as target variables calculated by the model. All of these parameters are automatically figured out by our system. The MapReduce execution parameters are gained from the job profiler. The hardware-related parameters are collected from the resource manager of YARN. The intermediate and target variables are automatically calculated according to the cost model.

Table 4.1: Variables in the cost model

\begin{tabular}{|l|l|}
\hline$T_{m}$ & Execution time of the map phase (sec) \\
$T_{r}$ & Execution time of the reduce phase (sec) \\
$P_{m}$ & Power consumption of the map phase (w) \\
$P_{r}$ & Power consumption of the reduce phase (w) \\
$T_{m}^{i o}$ & Waiting time of disk I/O in the map phase (sec) \\
$T_{m}^{p}$ & Processing time of the map functions (sec) \\
$T_{s}$ & Shuffle time of the reduce phase (sec) \\
$T_{r}^{i o}$ & Waiting time of disk I/O in the reduce phase (sec) \\
$T_{r}^{p}$ & Processing time of the reduce functions (sec) \\
$U_{m}$ & CPU utilization of nodes in the map phase \\
$U_{r}$ & CPU utilization of nodes in the reduce phase \\
$U_{s}^{m}$ & Switch utilization in the map phase \\
$U_{s}^{r}$ & Switch utilization in the reduce phase \\
$N$ & \# of nodes \\
$N_{s}$ & \# of Switches \\
$R$ & Physical resources per node (CPU, MEM) \\
$R_{m}$ & Physical resources required by each map task (CPU, MEM) \\
$R_{r}$ & Physical resources required by each reduce task (CPU, MEM) \\
$|I|$ & The size of the input data (MB) \\
$B_{d}$ & Disk bandwidth under MapReduce-like access patterns (MB/s) \\
$B_{n}$ & Network bandwidth (MB/s) \\
$S_{m}$ & Data selectivity of map tasks \\
$S_{r}$ & Data selectivity of reduce tasks \\
$B_{m}$ & Processing rate of user-defined map function (MB/s) \\
$B_{r}$ & Processing rate of user-defined reduce function (MB/s) \\
$h$ & A factor captures the extra disk I/O relative to the data read in per reduce task \\
\hline &
\end{tabular}

Job Profiling: It is generally difficult to estimate the cost of user-defined functions directly. Similar to the previous studies [102], we profile the MapReduce job by running it using a random sample of the input data (in our experiment, the number of map tasks is ten). The profiler gains the running 
time for each task and each phase, the amount of data consumed and produced by each task. All those statistics are gathered during execution, and can be used to decide the data selectivity of map/reduce function $\left(S_{m}, S_{r}\right)$ and the processing rate of map/reduce function $\left(B_{m}, B_{r}\right)$. For instance, the processing rate of the job's map function can be easily obtained as the ratio of the data consumed by individual map functions to the actual running time of map function, measured with Linux "time" command. We take the averages of the sample executions as our predictions.

Hardware Models: The hardware components include network switches and servers. Total power consumption of hardware consists of two types of power: static and dynamic consumption. Static consumption (including storage architecture, power supply, etc.) can be measured easily by keeping hardware in idle state. Here we mainly study the dynamic consumption. For the server, we use the model from the previous study [45]. The basic idea is to define a function $\mathfrak{f}(c)$ as the power of a server, where $c$ is the CPU utilization. For network switch, we adopt the power consumption model from a previous study [105]. It is also a utilization based model. The power of the switch is defined as $\mathfrak{g}(c)$, where $c$ is the switch utilization. For space interests, we omit the details on calibrating the model in our local cluster and refer readers for more details in the original papers $[45,105,106]$.

We model I/O and CPU processing of a MapReduce job execution with the following two phases.

Map Phase: We divide the map phase into two components: disk I/O and data processing.

For the disk I/O, each map task reads from local disk (assuming the input is locally available), and then also writes the intermediate data produced by map function to local disk. It is bounded by the effect of the disk bandwidth. Thus, we calculate the waiting time of disk I/O in the map phase $\left(T_{m}^{i o}\right)$ to be $\frac{|I| \times\left(1+S_{m}\right)}{N \times B_{d}}$.

For the data processing, each map task applies the user-defined map function on its input split. Thus, We calculate the processing time of the map functions $\left(T_{m}^{p}\right)$ to be $\frac{|I|}{N \times\left(R / R_{m}\right) \times B_{m}}$.

We assume that the CPU is idle during the disk/network I/O, the CPU utilization of the nodes in map phase $\left(U_{m}\right)$ is estimated to be $\frac{T_{m}^{p}}{T_{m}^{i o}+T_{m}^{p}}$. The switch utilization in the map phase $\left(U_{s}^{m}\right)$ is estimated to be zero. Hence, we calculate the execution time and power consumption of the map phase as follows, 


$$
\begin{array}{r}
T_{m}=T_{m}^{i o}+T_{m}^{p} \\
P_{m}=N \times \mathfrak{f}\left(U_{m}\right)+N_{s} \times \mathfrak{g}\left(U_{s}^{m}\right)
\end{array}
$$

Reduce Phase: The reduce phase is split into two components: data shuffling and reduce processing.

For the data shuffling, reduce tasks includes two operations. First, each reduce task reads its partition of the intermediate data across the network, and then merges and writes it locally. Thus, the bandwidth of this operation is the minimal value of $B_{d}$ and $B_{n}$, which is expressed as $\operatorname{Min}\left(B_{d}, B_{n}\right)$. Second, the data is read locally and an external merge is conducted before the data is consumed by the reduce phase. This operations is bounded by disk bandwidth $B_{d}$. We use a factor $h$ to capture the extra disk I/O relative to the data read in for a reduce task, which is obtained from job profiling. Thus, we calculate the shuffle time $\left(T_{S}\right)$ to be $\frac{|I| \times S_{m}}{N} \times\left\{\frac{1}{\operatorname{Min}\left(B_{n}, B_{d}\right)}+\frac{h}{B_{d}}\right\}$.

For the reduce processing, each reduce task reads its input from disk, applies the user-defined reduce function and writes the final result to disk. Similar to the map phase, we calculate the waiting time of disk I/O in the reduce phase $\left(T_{r}^{i o}\right)$ to be $\frac{|I| \times S_{m} \times\left(1+S_{r}\right)}{N \times B_{d}}$ and the processing time of the reduce functions $\left(T_{r}^{p}\right)$ to be $\frac{|I| \times S_{m}}{N \times\left(R / R_{r}\right) \times B_{r}}$.

The CPU utilization of the nodes in the reduce phase $\left(U_{r}\right)$ is estimated to be $\frac{T_{r}^{p}}{T_{s}+T_{r}^{i o}+T_{r}^{p}}$. Given the amount of data shuffled, we can easily derive the utilization $U_{s}^{r}$ for the switches involved. Hence, we calculate the execution time and power consumption of the reduce phase as follows,

$$
\begin{array}{r}
T_{r}=T_{s}+T_{r}^{i o}+T_{r}^{p} \\
P_{r}=N \times \mathfrak{f}\left(U_{r}\right)+N_{s} \times \mathfrak{g}\left(U_{s}^{r}\right)
\end{array}
$$

With these two phases modeled, the total execution time of the job is simply $T_{m}+T_{r}$, and the total energy consumed is $T_{m} \times P_{m}+T_{r} \times P_{r}$. 
As we will see in the experiments, the cost model is simple and lightweight, with only a small number of pre-executed tasks and very low computation overhead. This profiling process is done for large jobs only. Also, the cost model is sufficiently accurate in capturing the performance and energy consumption of MapReduce/Hadoop, and is effective in guiding the scheduling and optimizations in JouleMR.

\subsubsection{Server Power Management}

To reduce the state transition overhead, we develop a simple approach with data replication. At the beginning of a time slot, we determine the set of machines that will be on to process the workload. We maximize the input data chunks to those workloads when we choose the server. We develop a simple and effective approach. We greedily choose a server that has the largest number of input data chunks for the workload of the current slot, which has not been covered in the pre-selected servers. We repeat the process until the number of servers reached the limit, or all the input data chunks are covered. If all the input data chunks are covered, we simply choose the server with the largest number of input data chunks (no matter whether they are covered in the pre-selected servers). Given the selected servers, we now consider data replications or keeping more servers on to make sure all the input data chunks are in the selected servers. The more servers that we plan to transit to the S3 state, the higher probability that the data blocks needed to replicate (the more energy will be saved). In our implementation, we use a heuristic approach to determine whether we transit a server to the S3 state. We limit the accumulated data transfer time to be smaller than a predefined threshold of the slot length. That threshold should be reasonably small for a low overhead. In our experiments, we set this threshold to be $5 \%$ of the slot length. If the accumulated data transfer time exceeds the threshold, we stop the consideration of transiting a server to the $\mathrm{S} 3$ state. Otherwise, we transit a server to the $\mathrm{S} 3$ state, and evenly replicate all the data chunks from that server to the already selected servers. 


\subsection{Cost-effective Scheduling}

In order to leverage dynamic pricing to reduce the electricity cost of the cluster, we provide costeffective scheduling by extending JouleMR. We find that the algorithms proposed in green-aware scheduling (presented in Sections 4.3) can be extended to develop cost-effective optimizations with taking special consideration on the electricity cost. In the following, we mainly focus on the major differences to green-aware scheduling: cost-effective job transformations and battery assisted cheap brown shifting algorithm.

\subsubsection{Basic Energy-efficient Plan}

We use same approach used in green-aware scheduling to generate energy-efficient plan. Moreover, we take special consideration on the dynamic pricing which is able to guide our cost-effective scheduling. We apply the same type of variable grid energy pricing as used in GreenHadoop [57]. It assumes on-peak/off-peak pricing. The energy costs more during on-peak times and less during off-peak times. Figure 4.5 shows the variation of the energy prices charged by PSEG in New Jersey. In this price scheme, energy price is less between $11 \mathrm{pm}$ and 9am (off-peak times) compared with that between 9am ad 11pm (on-peak times). We apply the same energy prices scheme used in GreenHadoop: $\$ 0.08 / \mathrm{kWh}$ (off-peak) and $\$ 0.13 / \mathrm{kWh}$ (on-peak). The peak grid power is charged at $\$ 13.61 / \mathrm{kW}$. Similar to GreenHadoop [57], we monitor the average brown power consumption every 10-minutes and calculate peak brown power as the maximum of these average that has been reached so far. If the brown power consumption beyond this peak value in one time slot, the brown energy will be charged at peak price. In our thesis, we assume the self-generated green energy is free. However, our cost-effective optimization algorithm can be extended to handle the cases where renewable energy is charged [107].

By considering the joule efficiency, delaying workload and scheduling it with low-price brown energy cannot reduce the electricity cost all the time. Let us study two simple examples shown in 


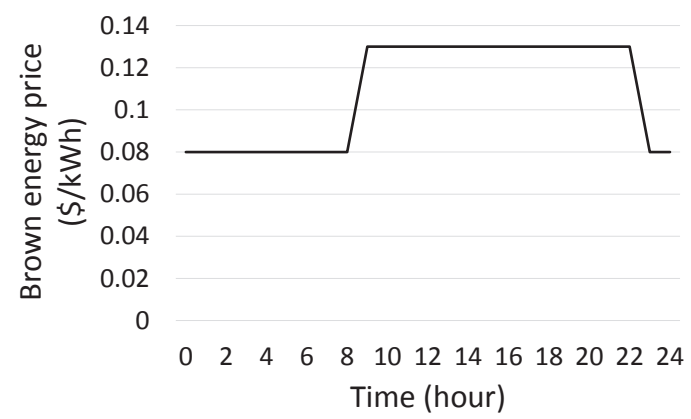

Figure 4.5: The price of brown energy over time

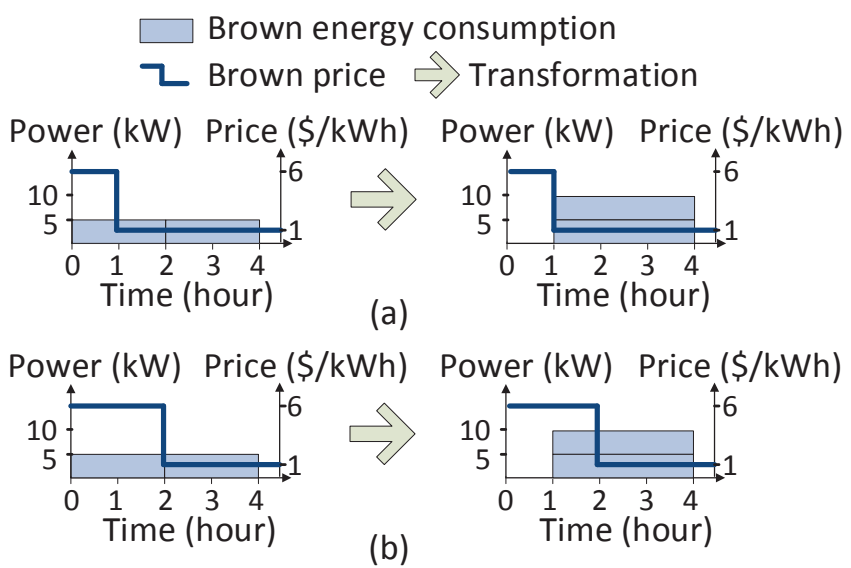

Figure 4.6: Two illustrative examples: (a) Delay and promote operation reduces the electricity cost; (b) Delay and promote operation increases the electricity cost.

Figure 4.6. If we execute the workload on one machine, its execution time is 4 hours and energy consumption is $20 \mathrm{kWh}$. On the other hand, if we execute it on two machines, due to the non-linear speedup feature of the workload, its execution time is 3 hours and its energy consumption is $30 \mathrm{kWh}$. In the case (a) of Figure 4.6, we delay the scheduling of the workload in order to utilize more lowprice brown energy, then the electricity cost can be reduced. On the contrary, in the case (b), executing the workload earlier even when the price of brown energy is high leads to a smaller electricity cost due to the brown energy is utilized more efficiently. This example shows that joule efficiency must be considered in order to effectively reduce the electricity cost of the cluster. 


\subsubsection{Cost-effective Optimizations with Job Transformations}

We perform further cost-effective optimizations on the basic energy-efficient execution plan by considering electricity prices and peak brown power charges. The basic idea of the cost-effective optimizations is scheduling workload with cheaper brown energy and shifting the peak brown consumption to the other time slots if the total electricity cost of the current epoch can be reduced.

Similar to the green-aware job transformations, we also utilize the 4 basic operators (Delay, Advance, Promote and Demote) to perform the job transformations on the basic energy-efficient plan. We extend the 2-phase algorithms (Algorithm 3 and Algorithm 4) to support the cost-effective optimizations by considering electricity cost of the execution plan in an epoch instead of the brown energy consumption. The electricity cost of the execution plan in an epoch is calculated as $\sum_{i=1}^{N} C_{i}$, where $N$ is the number of time slots in one epoch and $C_{i}$ is the electricity cost of time slot $i . C_{i}$ is defined as follows:

$$
C_{i}= \begin{cases}p_{i}^{g} \times J_{i}, & \text { if } p_{i}^{g}<=p_{i}^{b} \text { and } G_{i}>=J_{i} \\ p_{i}^{g} \times G_{i}+p_{i}^{b} \times\left(J_{i}-G_{i}\right), & \text { elif } p_{i}^{g}<=p_{i}^{b} \text { and } G_{i}<J_{i} \\ p_{i}^{b} \times G_{i}, & \text { elif } p_{i}^{g}>p_{i}^{b}\end{cases}
$$

, where $p_{i}^{g}$ is the price of green energy at time slot $i, p_{i}^{b}$ is the price of brown energy at time slot $i$, $J_{i}$ is the total energy consumption of all jobs running in time slot $i$ (estimated using the cost model), and $G_{i}$ is the predicted amount of green energy in time slot $i$. If the green energy is cheaper than the brown energy, we utilize the green energy with higher priority until it is used up. Otherwise, the brown energy is utilized.

\subsubsection{Battery Assisted Cheap Brown Shifting}

We develop our battery assisted cheap brown shifting as a further optimization for the execution plan optimized by the cost-effective job transformations. Our optimization is also performed at the beginning of each time slot. With the help of batteries, we can purchase the brown energy at off-peak period 
with lower price and use it at on-peak period. As there is energy loss during charging/discharging, we need to compare the electricity cost saving due to the price differences and the loss in the charging/discharging. Still, we can formulate this problem as a nonlinear integer programming problem with constraints, which is NP-hard. Therefore, we propose a heuristic algorithm to perform the cheap brown shifting on the execution plan.

Cheap Brown Shifting Algorithm: We iterate the execution plan in the ascending order. For each off-peak time slot, we consider purchasing the brown energy at lower price and charging it for future usage for on-peak hour. As not all of the purchased brown energy can be utilized in the future because of energy loss in charging/discharging. Therefore, we need to compare the electricity cost saving due to the on-peak/off-peak price differences with the loss due to the energy waste. If the benefit from the price differences is larger than waste during charging/discharging, we commit the purchasing and charging for future usage.

\subsection{Evaluation}

We evaluate JouleMR in a local small-scale cluster and a large-scale simulator in this section.

\subsubsection{Experiment Setup}

We evaluate JouleMR with two complementary sets of experiments. The first experiment is on a real testbed on a 10-node testbed with micro benchmarks including TeraSort in Hadoop APIs and GridMix ${ }^{1}$ benchmarks. This small-scale experiment in the real cluster is to reveal micro-level details by fully controlling the choices on the scheduling and to evaluate the energy consumption and performance models. The second set of our experiments is to perform simulations with the real-world trace comes from data centers (particularly from Facebook) to assess the effectiveness of JouleMR in large-scale systems.

\footnotetext{
${ }^{1}$ http://hadoop.apache.org/docs/stable/gridmix.html
} 
In both sets of experiments in the local cluster and simulations, the battery and solar panels are simulated. The battery charging efficiency is set to be 0.82 , according to our measurements on a Fujitsu lithium ion battery with $10.8 \mathrm{~V}$ voltage. The charging/discharging model for lithium ion battery can also be applied to lead-acid batteries but with different charging efficiencies. The leadacid batteries also account for the fact that only percentage of the energy charged will be available for later use [63]. Our model is general for both kinds of batteries. By default, the battery capacities are $1 \mathrm{kWh}$ and $20 \mathrm{kWh}$ for the experiments in the local cluster and simulations, respectively. We utilize the real-world traces for solar energy from Measurement and Instrumentation Data Center (MIDC), as solar energy is widely available. By default, we use the two days (May 1-2, 2011), as illustrated in Figure 4.7. The solar energy supply is intermittent due to the weather impact. The peak solar power supply is provisioned according to capacity of the cluster by default. The time slot size is one minute and the epoch size is 20 minutes.

We implement JouleMR based on top of the latest version of Hadoop (Hadoop YARN 2.6.0). We consider Hadoop YARN 2.6.0 as the baseline, denoted as "Hadoop". Hadoop uses the default scheduler, and jobs are scheduled for executions without delay. We choose the state-of-the-art green-aware Hadoop [57] (“GreenHadoop”) for comparison. Unlike JouleMR, GreenHadoop has not considered the joule efficiency of MapReduce or the energy efficiency of the battery usage. We have got the original source code of GreenHadoop and adapted GreenHadoop to run on Hadoop YARN. To evaluate the benefits of different optimization techniques more clearly, we enable/disable specific optimizations manually in both real testbed and simulations. We have three optimization variants: "JouleMR(BE)", "JouleMR(JT)" and "JouleMR". The detailed configurations of optimization techniques in all compared algorithms are summarized in Table 4.2. JouleMR has all the optimizations on joule efficiency proposed in this thesis. The difference between JouleMR(BE) and JouleMR(JT) measures the effectiveness of our job transformations. The difference between JouleMR(JT) and JouleMR measures the effectiveness of our battery assisted energy shifting optimization. Also, all algorithms use battery, 
either in the basic approach or with our proposed battery assisted energy shifting technique. In terms of the low and high utilization of the data center, JouleMR makes sure that the energy is utilized efficiently. We adaptively adjust the number of active machines according to the execution plan and the other machines are turned to low power state (i.e., ACPI S3), and use the replication method [57] to guarantee the data availability when a machine is set to the low power state. Our approach can work regardless of cluster utilization. We study the impact of cluster utilization in Section 4.5.4.

The runtime overhead of running JouleMR scheduler is small. In our experiment, that overhead for a typical MapReduce job is less than 0.1 millisecond on a single Intel Xeon X5675 CPU core. This is mostly ignorable for data-intensive workloads.

Table 4.2: Configuration of optimization techniques for all schedulers

\begin{tabular}{|c|c|c|c||c|c|}
\hline $\begin{array}{c}\text { Optimization } \\
\text { Scheduter }\end{array}$ & SDE & $\begin{array}{c}\text { Energy- } \\
\text { efficient } \\
\text { Plan }\end{array}$ & $\begin{array}{c}\text { Job Trans- } \\
\text { formation }\end{array}$ & $\begin{array}{c}\text { Basic } \\
\text { Battery } \\
\text { Usage }\end{array}$ & $\begin{array}{c}\text { Green } \\
\text { Shifting }\end{array}$ \\
\hline Hadoop & & & & $\checkmark$ & \\
\hline GreenHadoop & $\checkmark$ & & & $\checkmark$ & \\
\hline JouleMR(BE) & & $\checkmark$ & & $\checkmark$ & \\
\hline JouleMR(JT) & & $\checkmark$ & $\checkmark$ & $\checkmark$ & \\
\hline JouleMR & & $\checkmark$ & $\checkmark$ & & $\checkmark$ \\
\hline
\end{tabular}

\subsubsection{Micro Benchmark Setup}

The local cluster consists of 10 nodes, each with 12 CPU cores, 24 GB memory and 500G SATA disks. The idle and peak power consumptions of one machine are $150 \mathrm{w}$ and $280 \mathrm{w}$, respectively. The machines are connected with $10 \mathrm{~Gb} / \mathrm{sec}$ Ethernet. One node is configured as master, and the others are deployed as slaves. We use multiple meters to measure the energy consumption of the whole system, including machines and networking.

We assemble a micro benchmark based on the daily pattern of Google search workload [108], as illustrated in Figure 4.7. The solid line represents the solar energy $(\mathrm{kWh})$ while the dashed line represents the assembled workload (\#jobs/hour). At the peak load point, each core of all the machines have one task (either map or reduce) to execute. We consider four kinds of jobs: Terasort and GridMix 


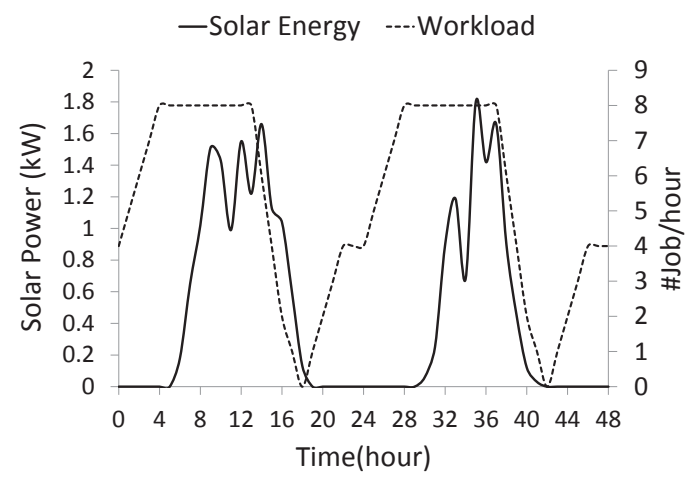

Figure 4.7: A snapshot of solar trace gained from MIDC, and a mixed workload simulating Google's search jobs

(WebdataSort, WebdataScan and APISort). By default, the input size of each job is around 80GB and the deadline is one hour. We consider a mixed workload, where each job is randomly generated from four kinds of jobs.

\subsubsection{Simulation Setup}

We develop a large-scale simulator with by taking as input the solar traces and production workload traces from data centers. We simulate the power-performance tradeoff model for the workload and charging/discharging life cycles of battery. In particular, we use a synthetic trace that models multiuser production workload in Facebook ${ }^{2}$ with a 600-node cluster. We generate the actual jobs with "loadgen" which is a configurable MapReduce job generating tool from the Gridmix benchmark included in the Hadoop distribution. The deadline of each job is computed based on its arrival time, expected execution time and slack. By default, the slack is set to $100 \%$ of the expected execution time. This setting is reasonable in practice, in the sense that users can tolerate a longer slack for a larger job. We also experimentally study other slack settings. The servers is configured with the same performance-energy consumption models as those in our local cluster. We apply two-level treestructured networks in our simulation: intra-pod switch with $1 \mathrm{~Gb} / \mathrm{sec}$ bandwidth, and inter-pod switch with $10 \mathrm{~Gb} / \mathrm{sec}$ bandwidth. Each pod has 32 machines.

\footnotetext{
${ }^{2}$ https://github.com/SWIMProjectUCB/SWIM/wiki
} 
Table 4.3: Facebook production trace characteristics

\begin{tabular}{|c|c|c|c|c|}
\hline Cat. & \% Energy & \% Jobs & \# Maps & \# Aver Reds \\
\hline 1 & $6 \%$ & $70.68 \%$ & {$[1,10]$} & 4 \\
2 & $4.7 \%$ & $10.73 \%$ & $(10,50]$ & 5 \\
3 & $4.6 \%$ & $4.61 \%$ & $(50,100]$ & 13 \\
4 & $5.03 \%$ & $7.02 \%$ & $(100,1000]$ & 116 \\
5 & $25.00 \%$ & $5.36 \%$ & $(1000,10000]$ & 595 \\
6 & $48.56 \%$ & $1.53 \%$ & $(10000,100000]$ & 5683 \\
7 & $6.11 \%$ & $0.06 \%$ & $(100000,1000000]$ & 8831 \\
\hline
\end{tabular}

We analyze the arrival pattern and energy consumption statistics of jobs with different sizes. Table 4.3 summarizes the statistics of the Facebook trace for 7 categories of jobs in different sizes. For each category, the 2nd column lists the fraction of energy consumption and 3rd column lists the fraction of the jobs; the 4th column lists the range of the number map tasks; the 5th column lists the average number of reduce tasks. Small jobs (\# Maps is smaller than 10) contributes to over $70 \%$ of the number of jobs, but their total energy consumption is less than $6 \%$. In contrast, the total energy consumption of the jobs with more than 1000 map tasks accounts for over $79 \%$ of the total energy usage. As discussed in Section 4.3.1, we develop a job size-aware prediction on performance and energy consumption. The arrival rate, energy consumption and execution time of small jobs is predicted with a time series algorithm, and large jobs are scheduled in an ad hoc manner.

\subsubsection{Green-aware Scheduling: Micro Benchmark Study}

\subsubsection{Model Accuracy}

In this section, we evaluate the accuracy of the cost model. We present the evaluation on the total energy consumption, since we have observed similar results on the performance. We evaluate the accuracy of our energy cost model by comparing energy consumption of individual jobs in our micro benchmark. We choose four kinds of jobs: Terasort and Gridmix (WebDataScan, WebDataSort and APISort). In our experiment, the inputs are generated uniformly by using TeraGen included in the Hadoop distribution and the data generators included in Gridmix benchmark. Figure 4.8 shows the estimated and measured energy consumption for running each job in the local cluster. For all jobs, 


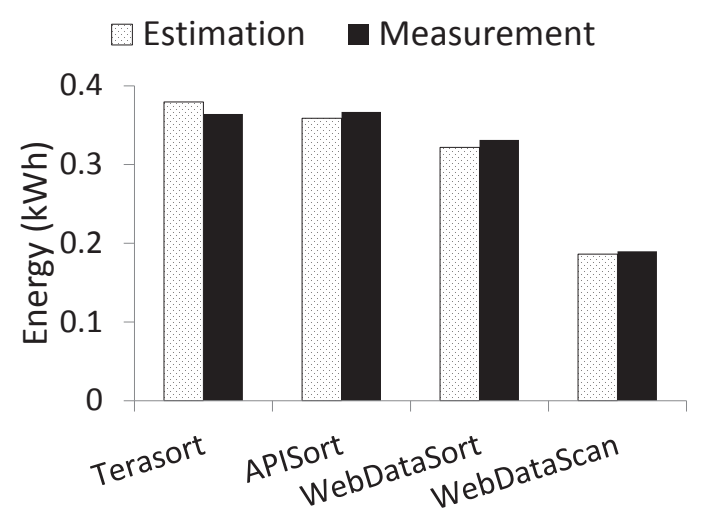

Figure 4.8: Estimated and measured energy consumption of individual jobs

the difference between estimation and measurement is smaller than $5 \%$ of the measurement when the number of profiling tasks is larger than 10 , which validates the accuracy of the energy consumption model. We also evaluate the average prediction for Terasort and Gridmix workload by varying the number of map task used for profiling. As we can see from the Table 4.4, our cost model achieves higher accuracy with the increase of map tasks to be profiled. Many dynamic factors including hardware failure, I/O contention and workload imbalance can result in inaccurate prediction, we study its impact to JouleMR in section 4.5 .4 by manually simulating the prediction error.

Table 4.4: Prediction error with different profiling overhead

\begin{tabular}{|l|l|l|l|l|}
\hline \# map task used for profiling & 5 & 10 & 20 & 40 \\
\hline average prediction error & $12.8 \%$ & $4.9 \%$ & $3.5 \%$ & $2.9 \%$ \\
\hline
\end{tabular}

\subsubsection{Overall Comparison}

Figure 4.9(a) shows the brown energy consumption of different schedulers in running the micro benchmark. Overall, both GreenHadoop and JouleMR consume less brown energy than Hadoop, thanks to the alignment between power demand and green supply. Compared with GreenHadoop, JouleMR still consumes less brown energy, by embracing the energy-efficient optimizations. JouleMR(BE) reduces the brown consumption by $7.1 \%$ compared with GreenHadoop by considering energy efficiency. JouleMR(JT) applies job transformations to the basic energy-efficient plan and further re- 
duces the brown consumption by $8.5 \%$ over JouleMR(BE). By integrating battery-assisted shifting, JouleMR reduces the brown consumption by $6 \%$ further over JouleMR(JT). We can see that the proposed approaches are orthogonal with each other in the optimization and result in accumulated energy reductions.

Figure 4.9(b) shows the energy breakdown. We breakdown the total energy usage into five parts: brown energy usage, green energy consumed by the workload directly, green energy charged into battery, green energy wasted and data replication overhead. Green energy wasted includes energy loss during charging/discharging and the discarded part when battery is fully charged. GreenHadoop can utilize more green energy than Hadoop by aligning power demand with green supply. However, without considering joule efficiency, it uses more brown energy than JouleMR. Particularly, JouleMR(BE) utilizes every joule of energy more efficiently and consumes less brown energy than GreenHadoop. Job transformations in JouleMR(JT) use more green energy in an efficient manner. Finally, battery assisted green shifting techniques in JouleMR charges more green energy into battery rather than using it directly, because some green energy can be utilized with higher energy efficiency in the future and thus further reduces the brown energy.

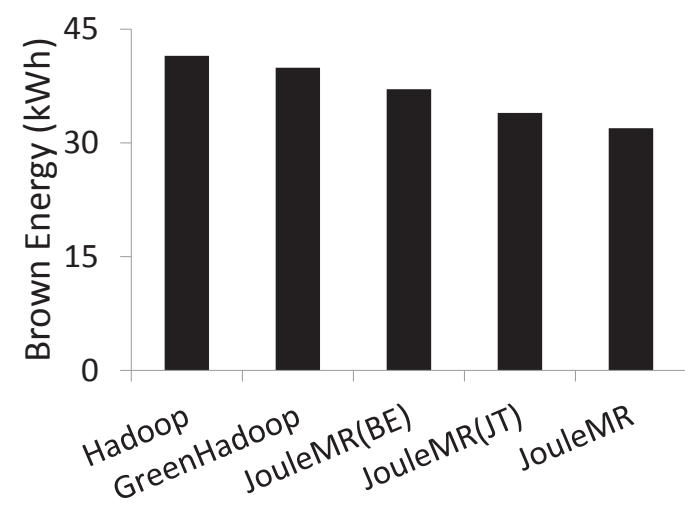

(a) Brown energy consumption

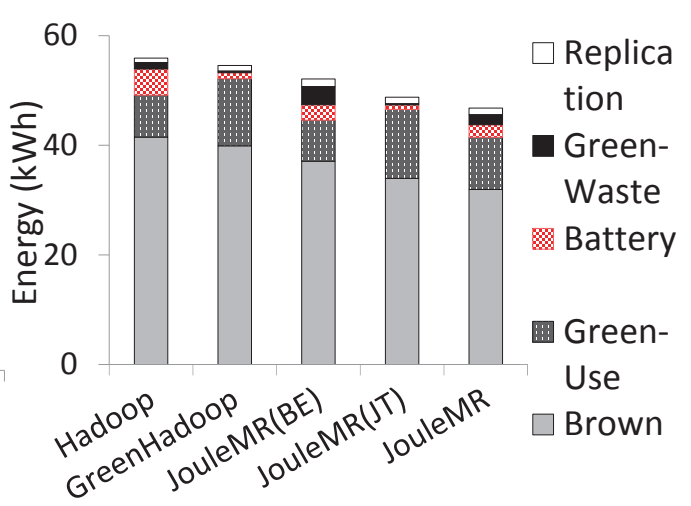

(b) Energy consumption breakdown

Figure 4.9: Overall comparison of green-aware optimizations with the micro benchmark

We further study the replication overhead. Because we take advantage of ACPI S3 to put servers to a lower power state for energy saving, some data blocks should be replicated to other servers 
for processing. We observed that the replication overhead is negligible (smaller than $20 \%$ of the energy saving by transiting the server to S3 when appropriate). Two more issues are worth further discussions. First, the state transition of a server is lightweight. The period of a server transiting from S3 back to the active state is around 7 seconds, and transiting from the active state to S3 takes only 1 second. Second, the network performance of our cluster is reasonably good. A data transfer of one HDFS chunk of $128 \mathrm{MB}$ takes less than 0.3 second in our local cluster.

\subsubsection{Green-aware Scheduling: Large-scale Trace Study}

First, we evaluate the accuracy of our large-scale simulator, followed by the detailed results on the production trace.

\subsubsection{Simulation Validation}

We use the SWIM workload replay tool to scale down Facebook production trace for our local cluster. With a similar scale-down approach in the previous study [57], we scale down the workload as follows: we reduce the data by a factor of 8 , and eliminate the largest $1.9 \%$ of jobs. We schedule the scaled-down workload in our local cluster and validate the simulation result with the real-measured value. Figure 4.10 shows the measured and simulated energy consumption. The difference between simulation and measurement is small (less than $2 \mathrm{kWh}$ ), which validates the accuracy of our simulator.

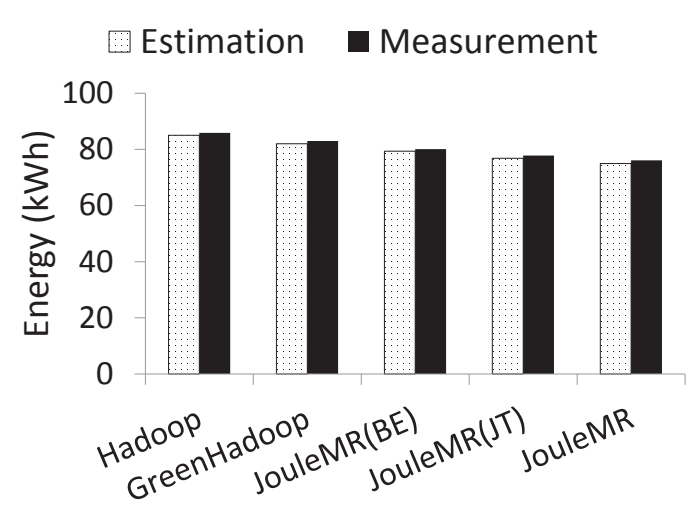

Figure 4.10: Validation of the green-aware simulations on replayed Facebook trace 


\subsubsection{Overall Comparison}

Figure 4.11(a) shows the brown energy consumption result on Facebook production workloads under five schedulers. The brown energy reduction of JouleMR is even larger than that in the local cluster, by $35 \%$ and $28 \%$ over Hadoop and GreenHadoop, respectively. Moreover, JouleMR has a lower total energy consumption, by $21 \%$ and $19 \%$ smaller than Hadoop and GreenHadoop, respectively. That shows the importance of integrating energy efficiency into the system.

The energy consumption breakdown is shown in Figure 4.11(b). In the experiments, we have observed similar results to the local cluster. We highlight with the following observations for simulations with the production workloads. First, the replication overhead is more significant than that of the local cluster, because data replications tend to be more frequent on a large-scale cluster. However, it is still kept in a reasonable low level (less than $5 \%$ of the total energy usage). Second, the battery plays a more important role in the large-scale cluster especially for the variants of JouleMR, because green energy is utilized with higher efficiency and more surplus green energy can be charged into battery. Meanwhile, as higher green supply and battery capacity are provisioned in our simulation, Figure 4.11(b) shows more green energy waste for the simulation study than our micro benchmark. Third, we study the detailed energy consumption along the timeline. All variants of "JouleMR" significantly reduce the peak usage of brown energy, in comparison with GreenHadoop.

Figure 4.12 shows the detailed energy consumption for Facebook production trace for GreenHadoop, JouleMR(BE), JouleMR(JT) and JouleMR. We can see 1) All variants of "JouleMR" significantly reduce the usage of brown energy, in comparison with GreenHadoop. 2) Even though JouleMR(BE) consumes less green energy compared to GreenHadoop, the consumption of brown energy is still reduced by considering energy efficiency. 3) JouleMR(JT) applies job transformations to utilize green energy better, therefore, the brown consumption is further reduced. 4) JouleMR reduces the brown consumption the most, because battery-assisted optimization charges the current available green energy into battery if it can be utilized more efficiently. 


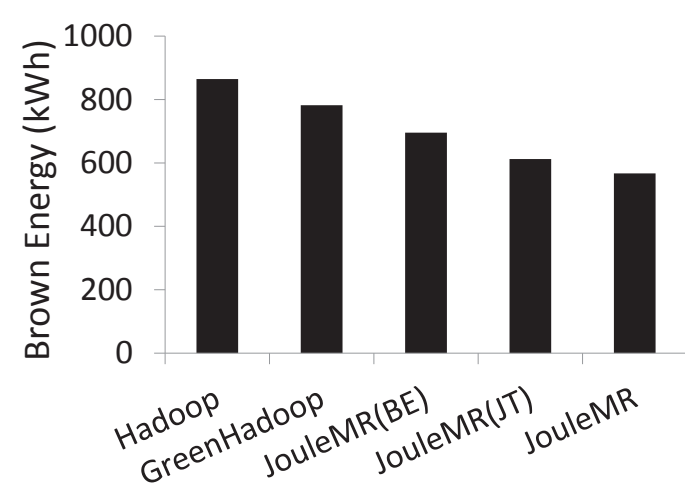

(a) Brown energy consumption

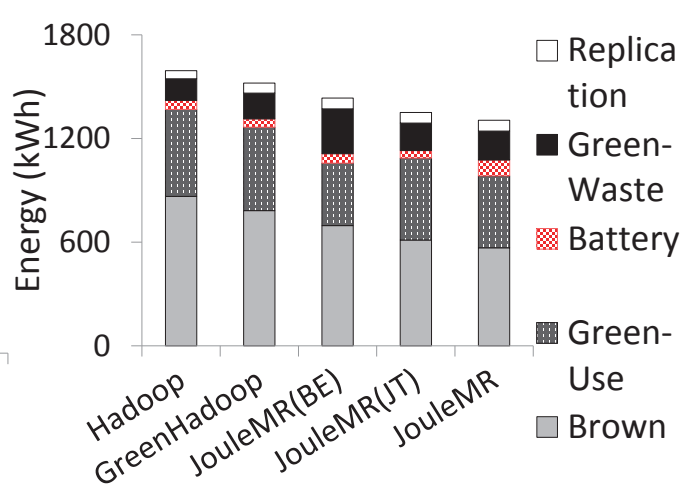

(b) Energy consumption breakdown

Figure 4.11: Overall comparison of green-aware optimizations with the Facebook production workloads

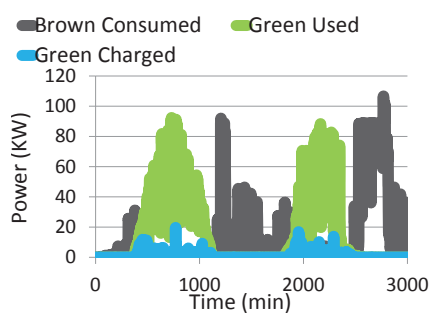

(a) GreenHadoop

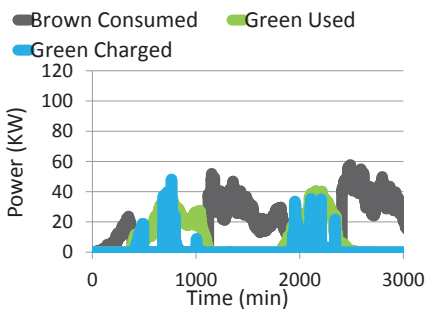

(b) JouleMR(BE)

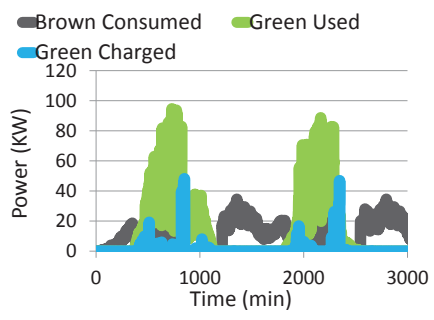

(c) JouleMR(JT)

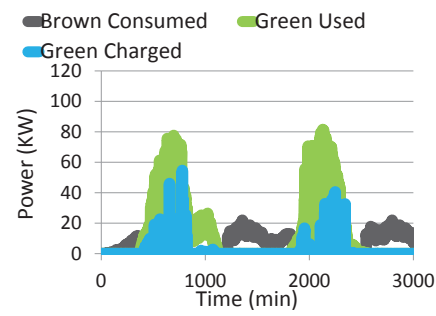

(d) JouleMR

Figure 4.12: Detailed energy consumption of different green-aware optimizations

\subsubsection{Sensitivity Studies}

In this section, we perform sensitivity studies on the parameters involved in JouleMR. Overall, JouleMR outperforms other schedulers in all different settings on those parameters. We present the results mainly with Facebook production trace.

Impact of Battery Capacity. Figure 4.13 shows the result by varying the battery capacity. The brown energy consumption of all schedulers decreases significantly at the beginning and finally becomes stable later as the increasing of the battery capacity. The increasing of the battery capacity has more effect for the variants of JouleMR, because more green energy can be saved and charged into battery by applying optimizations considering joule efficiency. Among all optimizations, battery 
assisted green shifting benefits the most from the increase of battery capacity.

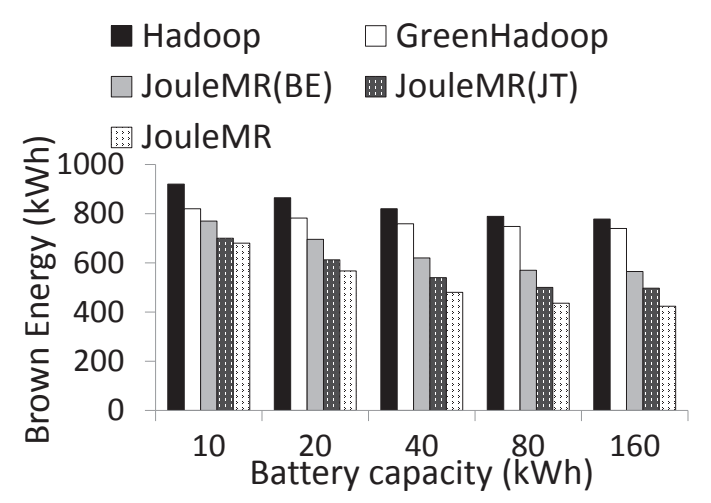

Figure 4.13: The brown energy usage varying the battery capacity

Impact of Slacks. Figure 4.14 shows the result of different slacks for Facebook production trace. We vary the slack in proportion to the default setting, in addition to setting the same deadline setting (one day) for all jobs used in GreenHadoop [57]. When the slacks increase, the opportunity to utilize green energy also increases, and thus energy can be utilized with higher efficiency. Regardless of different deadline settings, JouleMR can reduce the brown consumption significantly compared to GreenHadoop, due to the optimizations considering joule efficiency. JouleMR(BE) performs worst and stays stable when the slacks is larger than $400 \%$. The reason is that JouleMR(BE) only considers the energy efficiency rather than the supply of green energy. As the increase of the slacks, the execution plan generated by JouleMR(BE) varies for higher energy efficiency at the beginning, and remains unchanged when the slacks reaches a threshold.

Impact of Energy Estimation. Figure 4.15 shows the brown energy consumption under different energy prediction errors. Specifically, given the real energy usage $w$ and a prediction error $e$, the estimation is randomly distributed in $[w, w(1+e)]$ in Figure 4.15(a) and is distributed randomly between $[w(1-e), w(1+e)]$ which is shown in Figure 4.15(b). The results demonstrate the robustness of our optimizations, if the prediction error is acceptable (less than $30 \%$ in our evaluation). We observed similar results on the prediction errors of execution time. 


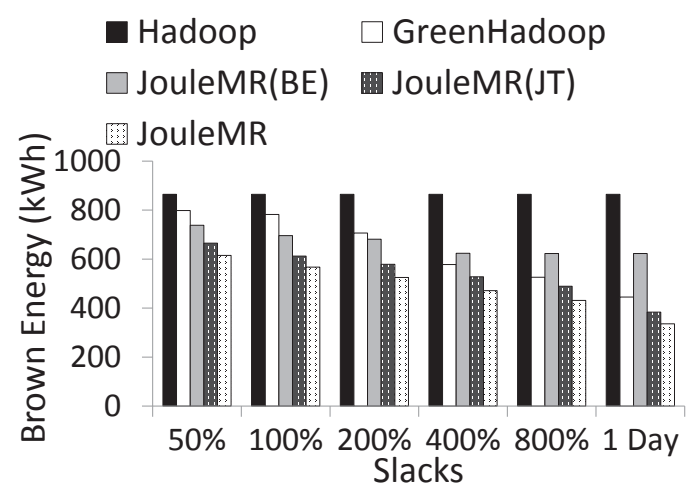

Figure 4.14: The brown energy usage varying slacks

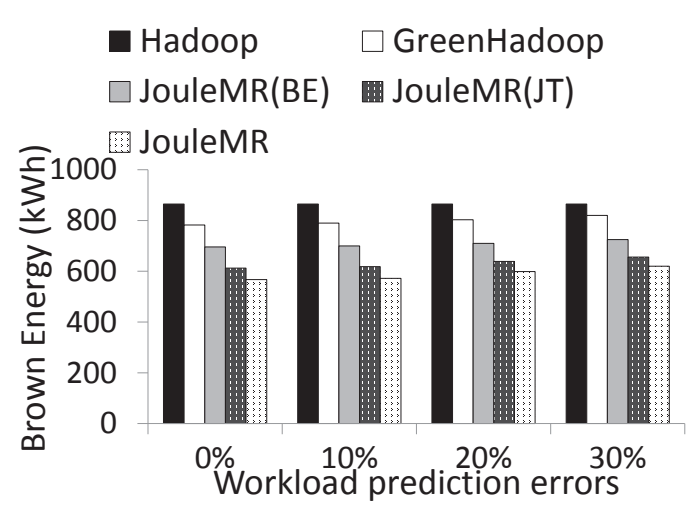

(a) Positive Error

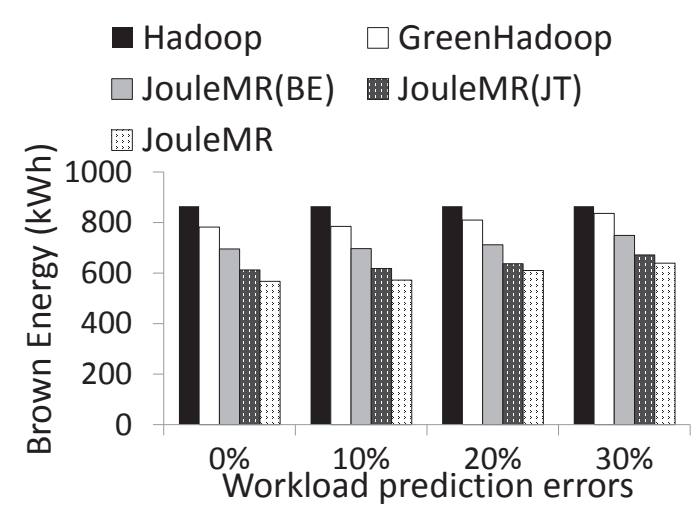

(b) Random Error

Figure 4.15: The brown energy usage varying workload prediction errors

Impact of Varying the Scale of Solar Energy. Figure 4.16 shows the brown energy usage by varying the scale of the solar energy supply. The scale can be increased by provisioning more number of solar panels and/or by increasing the area of solar panels. With the increases of the scale of the solar energy, the brown energy consumption of all approaches decreases and the brown energy usages are getting closer due to more workloads can be scheduled with green energy. However, in practice, the peak power of green energy is provisioned at most to the peak power usage of the cluster. JouleMR consumes more energy than other approaches when the scale of solar energy is larger than 400\%, because it does not aware the distribution of green energy and only executes following the generated plan. 


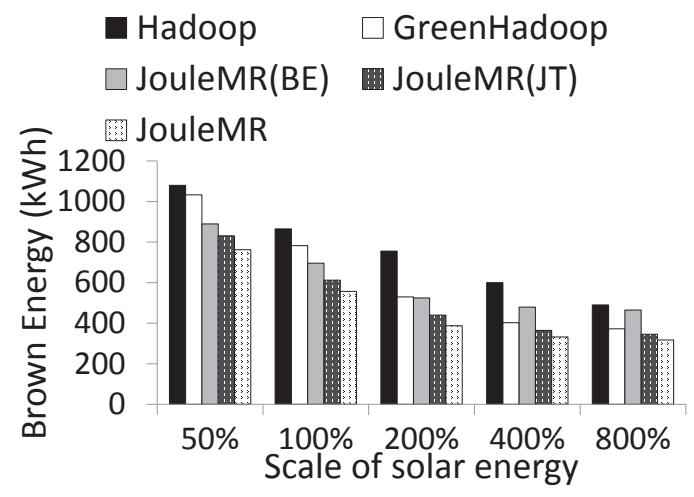

Figure 4.16: The brown energy usage varying the scale of solar energy

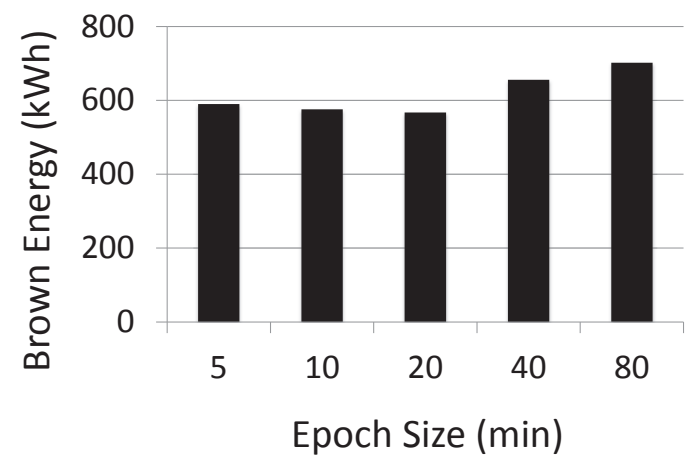

Figure 4.17: The brown energy usage varying the epoch size

Impact of varying epoch size in scheduling. Figure 4.17 shows the result by varying epoch sizes in scheduling. The brown energy consumption decreases slightly at the beginning with the increase of the epoch size (from 5 mins to 20 mins) as the scheduling plan benefits from the more future information which can be predicted accurately. After that, with the increase of the epoch size (from 20 mins to 80 mins), the brown energy consumption increases significantly due to the less accurate prediction as well as less robustness. Thus, this thesis chooses 20 minutes as a suitable epoch size.

Effects of JouleMR under highly variable solar energy. The trace we used in the evaluation contains the highly variant case due to the impact of the weather and other factors and the trace is shown in Figure 4.18(a). We synthesize a workload with the trace in the trace-driven simulator according to the length of this choosing period and schedule them with different schedulers. Figure 4.18(b) shows that JouelMR still performs efficiently even in the highly variably trace and the 
battery becomes more important when the solar energy becomes more variable.

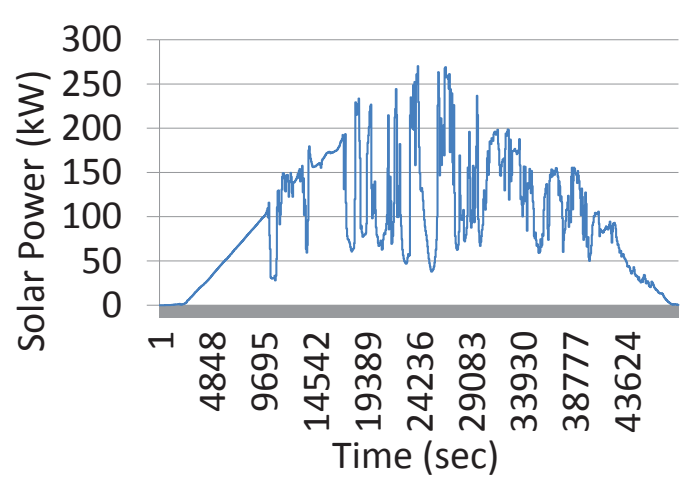

(a) Highly variable trace

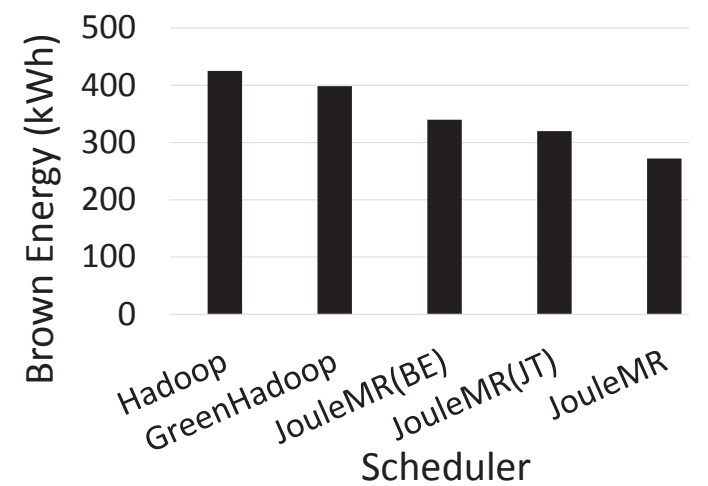

(b) Brown energy consumption

Figure 4.18: Brown energy usage when solar trace is highly variable

Impact of cluster utilization. Figure 4.19 shows the impact of different data center utilizations by varying the cluster size. In term of different data center utilizations, JouleMR performs effectively compared to Hadoop and GreenHadoop. The improvement increases with the increase of the cluster size (i.e., with the cluster resource utilization decreases).

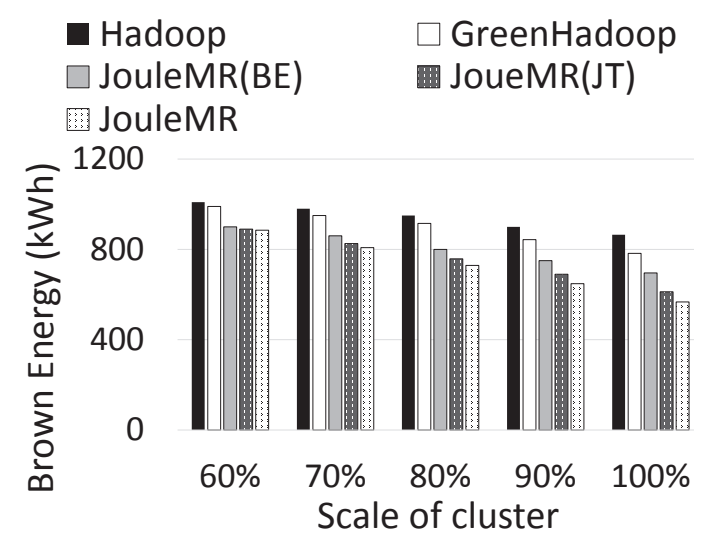

Figure 4.19: The brown energy usage varying the cluster size 

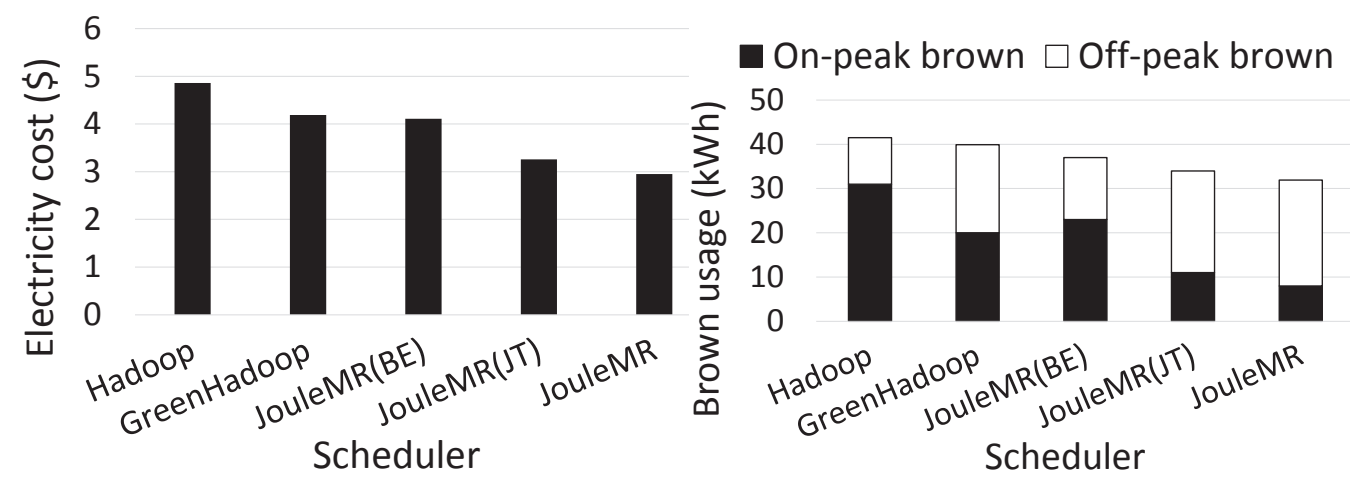
(a) Electricity cost of different schedulers
(b) Brown energy usage breakdown (on- peak/off-peak)

Figure 4.20: Overall comparison of cost-effective optimizations with the micro benchmark

\subsubsection{Results on Cost-effective Scheduling}

Micro Benchmark Study. Figure 4.20(a) shows the electricity cost of different schedulers in running the micro benchmark. Overall, both GreenHadoop and JouleMR have smaller electricity cost than Hadoop, since they have special considerations on the variation of the price. Compared with GreenHadoop, JouleMR still reduces the electricity cost further, by embracing the energy-efficient optimizations. JouleMR(JT) applies cost-effective job transformations to the basic energy-efficient plan and further reduces the electricity cost. By integrating batter-assisted cheap brown shifting, JouleMR further reduces the electricity cost over JouleMR(JT). Figure 4.20(b) shows the brown usage breakdown. It shows the amount of brown usage at on-peak/off-peak times. JouleMR maximizes the usage of brown energy during off-peak periods and minimizes the usage of brown energy during on-peak periods.

Large-scale Trace Study. Similar to simulation validation in the green-aware scheduling, we also shows the accuracy of our simulator in the context of cost-effective optimizations. Figure 4.22 shows the measured and simulated electricity cost in our local cluster. The difference between simulation and measurement is very small which validates the accuracy of our simulations.

Figure 4.21(a) shows the electricity cost on Facebook production workload under different sched- 


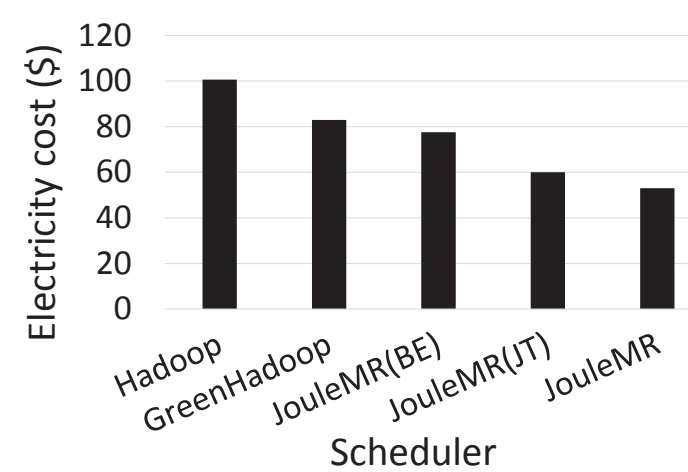

(a) Electricity cost of different schedulers

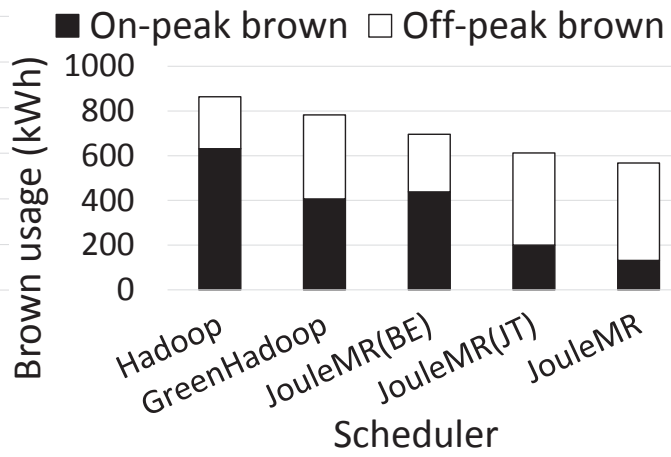

(b) Brown energy usage breakdown (onpeak/off-peak)

Figure 4.21: Overall comparison of cost-effective optimizations with the Facebook production workloads

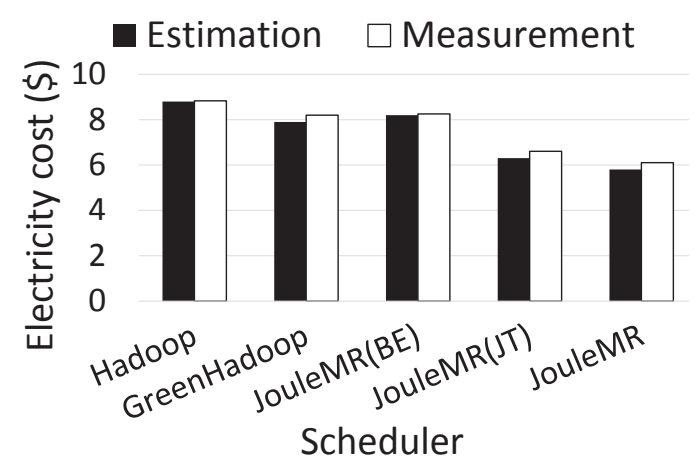

Figure 4.22: Validation of the cost-effective simulations on replayed Facebook trace

ulers and the on-peak/off-peak brown usage breakdown is shown in Figure 4.21(b). We find that our cost-effective scheduling still outperforms the state-of-the-art approaches in the large-scale environment.

Figure 4.23 shows the detailed usage of brown energy overtime. JouleMR not only matches the low-price brown energy with the workload demand very well but also utilizes the brown energy efficiently. That shows the effectiveness of leveraging joule efficiency with dynamic pricing schemes. 


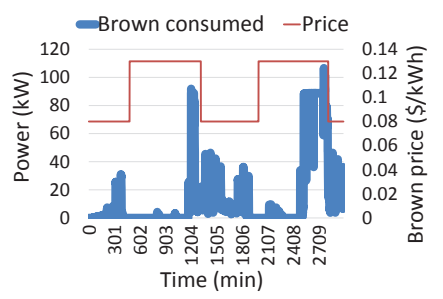

(a) GreenHadoop

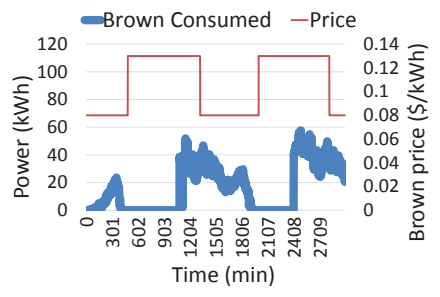

(b) JouleMR(BE)

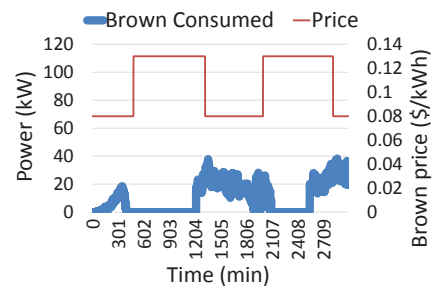

(c) JouleMR(JT)

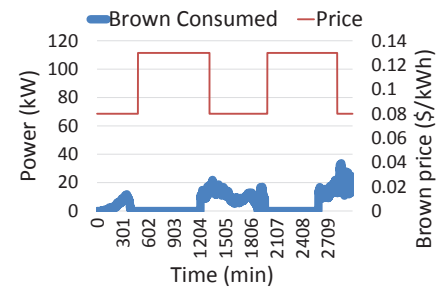

(d) JouleMR

Figure 4.23: Detailed brown usage of different cost-effective optimizations

\subsection{Conclusion}

This study proposes JouleMR by integrating green-aware and cost-effective job/task scheduling into MapReduce. It considers the key aspects of joule efficiency in a MapReduce cluster, including energy efficiency of MapReduce workloads, renewable energy supply, dynamic pricing schemes and battery usage. An analytical model is developed to guide scheduling decisions. We have implemented JouleMR on top of Hadoop. Our results show that JouleMR reduces the brown energy significantly on both real experiments and simulations compared with GreenHadoop [57] (up to 35\% and 28\% reduction, respectively). Besides, JouleMR reduces the electricity cost on both real experiments and simulations compared to GreenHadoop (by $30 \%$ and $36 \%$ reduction, respectively). 


\section{Chapter 5}

\section{Multi-objective Optimizations in Geo-distributed Data Analytics Systems}

In this chapter, we present the system GeoSpark, a job scheduler which supports multiple-objective optimization on geo-distributed data analytics systems.

\subsection{Introduction}

With the distribution of big data on multiple geographically datacenters (DCs), it has become more and more popular to deploy big data analytics jobs onto multiple geo-distributed DCs. On one hand, such deployment can provide low service access latency to users around the world. For example, major cloud providers like Amazon, Microsoft and Google each deploys tens to hundreds of geodistributed DCs world-wide $[109,110,111]$. On another hand, due to the security and privacy constraints, some data are not allowed to be moved around to other DCs $[112,113]$ and thus have to be processed distributedly. Applications such as web search, product recommendation, advertisement and business analysis generate huge volumes of data (e.g., query logs, url clicks, user behaviors) everyday at different DCs. It would be extremely expensive to move those data across DCs to perform centralized analytics $[113,114]$. Thus, it is more efficient and sometimes inevitable to perform geo-distributed data analytics. 
Recently, we have witnessed many geo-distributed data analytics systems are designed and developed for this geo-distributed environment, such as Iridium [38], SWAG [39], Spanner [115] and JetStream [116]. Most of those studies only consider optimizing a single objective, including resource efficiency [113, 114, 38], job latency [39, 116] and fairness [39]. However, modern job schedulers and resource coordinators in data processing frameworks often need to consider multiple objectives simultaneously due to various system operators' requirements on data analytics. More importantly, these objectives can be translated to discordant actions [73] and thus make the multi-objective optimization quite challenging. For example, a fair scheduler such as DRF [13] may decide to give resources to a certain job while the efficiency-aware heuristic that maximizes resource efficiency may prefer a different job with better data locality. Finding an optimal scheduling plan for multiple discordant objectives is very costly, especially for production workload in large-scale geo-distributed data analytics systems [117].

Distributed data analytics in the geo-distributed data analytics systems poses new challenges to the multi-objective optimization problem due to the special features of geo-distributed DCs.

First, the network performance in the geo-distributed data analytics systems is highly heterogeneous. Different DCs are connected using a congestion-free network in the geo-distributed environment, and the performance bottlenecks are only between the DCs and the core $[118,38]$. Additionally, the bandwidths of uplinks and downlinks between the DCs and the core are heterogeneous due to the widely different link capacities and the applications sharing these links. For example, Table 5.1 and 5.2 show the upload and download bandwidths between Amazon EC2 and Windows Azure clouds and the Internet, respectively. The upload bandwidth can be $8 \mathrm{X}$ as high as the download bandwidth. This feature of the geo-distributed data analytics systems can greatly affect some optimization objectives such as fairness and job latency while impacting less other objectives. Thus, it is important to study the impact of those features of the geo-distributed data analytics systems to different optimization objectives. 
Table 5.1: Uplink/downlink bandwidths (GB/s) of cc2.8xlarge instances from three Amazon EC2 regions to the Internet.

\begin{tabular}{|c|c|c|c|}
\hline & US East & AP Singapore & AP Sydney \\
\hline Uplink Bandwidth & 0.52 & 0.55 & 0.48 \\
\hline Downlink Bandwidth & 2.8 & 3.5 & 2.5 \\
\hline
\end{tabular}

Table 5.2: Uplink/downlink bandwidths (MB/s) of H8 instances from three Windows Azure cloud regions to the Internet.

\begin{tabular}{|c|c|c|c|}
\hline & US East & West Europe & Japan East \\
\hline Uplink Bandwidth & 64 & 42 & 36 \\
\hline Downlink Bandwidth & 485 & 210 & 44 \\
\hline
\end{tabular}

Second, data movement in the geo-distributed data analytics systems is constrained. On one hand, the security and privacy regulations prevent moving sensitive data around. On another hand, the high data movement cost due to the scarce cross-DC network bandwidths make it less preferable to move data across DCs. The original dataset is processed locally and the intermediate data needs to transfer across DCs. Thus, the data locality of at the DCs level becomes more important especially when these is substantial skew across DCs in the intermediate data of a job. Such constraints further complicate the job scheduling and resource coordination problems in the geo-distributed data analytics systems.

To address the challenges above, we propose GeoSpark, an extension of Apache Spark which is an efficient and general data processing framework commonly used in large-scale clusters, to support multi-objective optimizations for the geo-distributed data analytics systems. We perform studies on the key features of the geo-distributed data analytics systems and explore the trade-off between different optimization objectives. Based on the obtained insights, we give the formal definitions of resource efficiency, job latency and fairness for the geo-distributed data analytics systems and formulate the multi-objective optimization problem mathematically. We convert the multi-objective optimization problem to a single-objective optimization using weighted-sum approach, where weights reflect system operators' preferences on different objectives. Our optimization problem is actually a special case of the Generalized Assignment problem (GAP) which has been proven to be APX-hard [117]. 
Solving this problem is costly especially for production workload in the geo-distributed data analytics systems. Thus, we model this scheduling problem as a bipartite graph matching problem and propose a efficient maximum weight matching algorithm to solve it.

We implement GeoSpark on top of Apache Spark and conduct testbed evaluation in our emulated geo-distributed environment with network performance measured in the real geo-distributed DCs. Evaluation using synthetic workloads shows that GeoSpark effectively performs the multi-objective optimizations based on system operators' preferences on different objectives. GeoSpark achieves up to $30 \%$ makespan reduction, $28 \%$ job latency reduction and better fairness guarantee compared with existing schedulers in Apache Spark in the geo-distributed DCs. We also open-source our implementation here: https://github.com/hustnn/GeoSpark.

In summary, we make the following contributions.

- We analyze the trade-off between multiple optimization objectives, including resource efficiency, job latency and fairness, for the geo-distributed data analytics systems.

- We formulate the multi-objective optimization problem mathematically and propose an efficient online heuristic algorithm to perform the multi-objective optimization for the geo-distributed data analytics systems.

- We implement GeoSpark, an efficient geo-distributed data analytics systems, on top of Apache Spark and the evaluation result shows that geoSpark can perform multi-objective optimization efficiently.

\subsection{Background and Motivation}

We first present an overview of job scheduling in geo-distributed DCs. Next, we illustrate the trade-off between discordant objectives in the geo-distributed setting with motivating examples. 


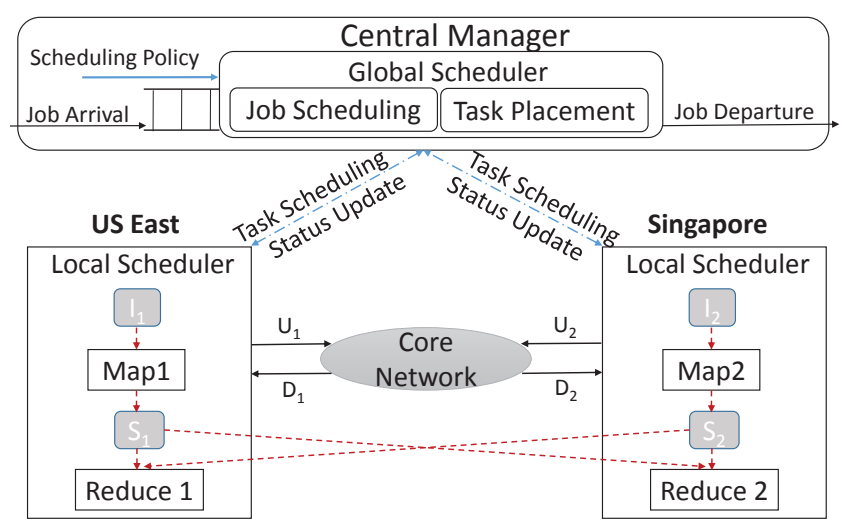

Figure 5.1: System architecture of geo-distributed job scheduling

\subsubsection{Job Scheduling in Geo-distributed DCs}

System Architecture. Figure 5.1 depicts the general framework for the geo-distributed data analytics. Similar to the architecture of the geo-distributed data analytics framework in Microsoft [38], we assume that the DCs are physically isolated at different geographic locations and are connected using a network with congestion-free core. The network performance bottlenecks are thus the uplinks and downlinks between DCs and the core [118]. Due to the widely different link capacities and the interferences from other applications sharing the same links, there can be significant heterogeneity in the network bandwidths. We use $U_{i}$ and $D_{i}$ to denote the uplink and downlink bandwidths of DC $i$ assigned to one job, respectively. We also assume that each DC has relatively abundant resources for computation.

Our geo-distributed data analytics system consists of a central manager and a set of local schedulers residing on each DC. When a job arrives, the central manager decides the job scheduling and task placement plan for all tasks in the job, using the scheduling policy specified by system operators and system status such as network bandwidths gathered from local schedulers. The local schedulers are responsible for executing tasks according to the optimized plan.

Analytics Jobs. An analytics job is a DAG consists of a set of stages (e.g., Map and Reduce). The tasks of input stage (e.g., map tasks) are executed on DCs with their input data, and write their 
outputs (e.g., intermediate data) locally to take advantage of data locality for better performance. Tasks of other intermediate stages of the job, such as reduce and join, can be placed at other DCs for efficient transfer of intermediate data across DCs. We use $I_{i}^{j}$ and $S_{i}^{j}$ to denote the amount of input and intermediate data of job $i$ at DC $j$, respectively.

In the geo-distributed setting, the input stage is extremely quick as a result of data locality [24] and efficient in-memory caching [37], and the latency of data analytics jobs is mainly dominated by intermediate stages which involve data communication on the Wide Area Network (WAN) links between different DCs [38]. For data-intensive applications, different placements of intermediate tasks can lead to different amounts of cross-DC data transfer [29, 119, 38]. Thus, it is important to carefully schedule those tasks to reduce the amount and time of intermediate data communication.

In the following, we consider analytics jobs with two stages, namely map and reduce stages for illustration purposes. We execute data analytics jobs using Apache Spark data processing framework, which is initially designed for a single DC.

Objectives. We mainly consider three objectives in this paper, namely resource efficiency, job latency and fairness. The three objectives are usually discordant with trade-offs between each other. The goal of this study is to provide a framework that can efficient perform multi-objective optimization according to the weights of different objectives. Formal definitions of the three objectives are presented in Section 5.3.

- Resource efficiency. The resource efficiency represents system throughput. In the geo-distributed data analytics system, the cross-DC data transfer is the most costly part, thus we utilize the data locality at DC level to quantify the resource efficiency.

- Job latency. We quantify the latency of a job at runtime, using the the number of running tasks and the number of unfinished tasks. 


\begin{tabular}{|c|c|c|c|}
\hline Parameter & D1 & D2 & D3 \\
\hline Reduce Input (MB) of Job 1 & 240 & 30 & 30 \\
\hline Reduce Input (MB) of Job 2 & 30 & 240 & 30 \\
\hline Uplink (MB/s) & 100 & 100 & 100 \\
\hline Downlink (MB/s) & 100 & 10 & 100 \\
\hline
\end{tabular}

Table 5.3: Workload information and configuration of the geo-distributed cluster.

- Fairness. The fairness provides the isolation guarantee for multiple jobs running concurrently in a shared environment. Due to the heterogeneous network bandwidths in geo-distributed DCs, we apply the definition of Dominant Resource Fairness (DRF) [13] and define the bottleneck link as the dominant resource of a task. Thus, we calculate the fairness of resource allocation using the resources allocated from the bottlenecked link.

\subsubsection{Motivating Examples}

In this subsection, we illustrate the importance of being aware of multiple discordant objectives for job scheduling in the geo-distributed setting with intuitive examples. As discussed above, there are two main features that affect the job scheduling results in the geo-distributed data analytics systems, namely the intermediate data distribution and the heterogeneous network bandwidths.

\subsubsection{Intermediate Data Distribution for trade-off (resource efficiency vs. fairness)}

Consider two MapReduce jobs with intermediate data (input of reduce tasks) distributed in three DCs $D 1, D 2$ and $D 3$ as shown in Table 5.3. The intermediate data of job 1 is highly skewed in $D 1$ and the intermediate data of job 2 is highly skewed in D2. As shown in Figure 5.2(a) and 5.2(b), task scheduling with consideration of the input data distribution can greatly reduce the amount of crossDC data transfer. An efficiency-aware scheduler may prefer to place the reduce tasks of job 1 in $D 1$ and the tasks of job 2 in $D 2$ to achieve good data locality and resource efficiency. However, a fair scheduler may choose tasks from jobs with the least resource allocation. 


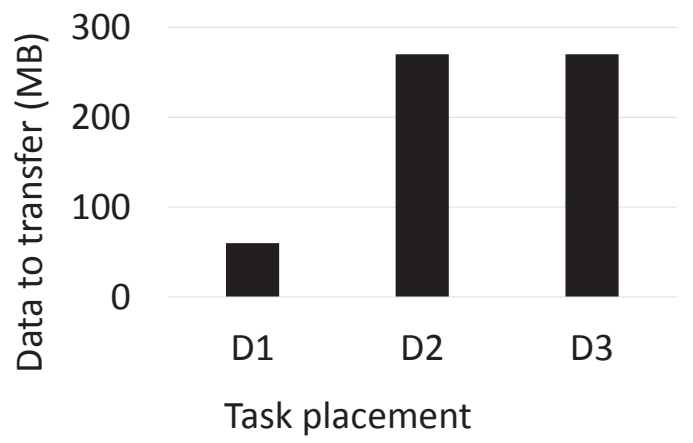

(a) Job 1

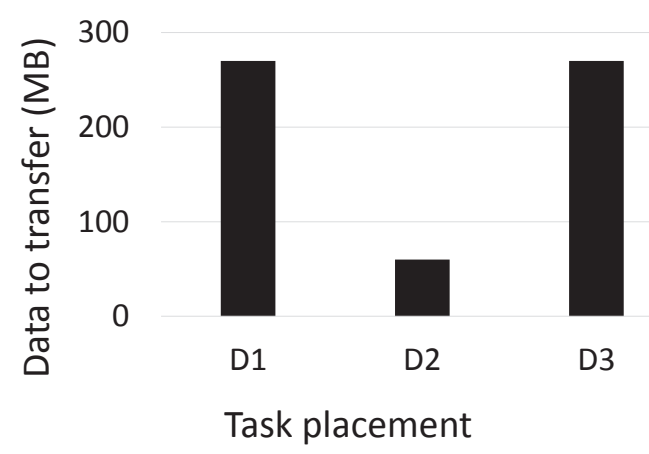

(b) Job 2

Figure 5.2: The amount of cross-DC data transfer for different placement of one reduce task.

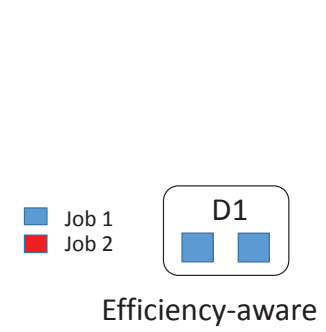

(a) Job assignment of different schedulers

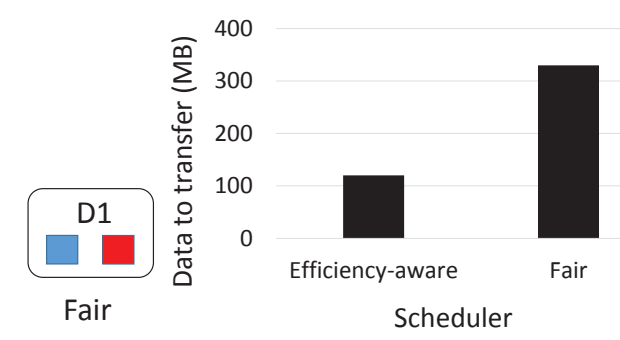

(b) Resource efficiency

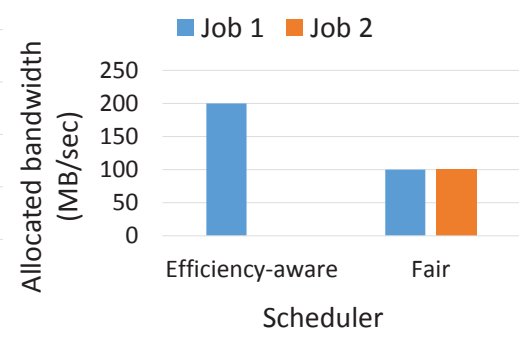

(c) Fairness

Figure 5.3: Illustration example of the trade-offs between resource efficiency and fairness impacted by the intermediate data distribution (Two free slots in $D 1$, efficiency-aware scheduler assigns 2 tasks of job 1 to $D 1$ and fair scheduler assigns 1 task of job 1 and 1 task of job 2 to $D 1$.)

Assume two slots in $D 1$ become available. The efficiency-aware scheduler schedules two tasks of job 1 to $D 1$ for good data locality. On the contrary, the fair scheduler schedules one task of job 1 and one task of job 2 to the slots, as shown in Figure 5.3(a). As shown in Figure 5.3(b), the efficiency-aware scheduler leads to less data transfer compared to the fair scheduler. At the cost, the efficiency-aware scheduler does not allocate resources fairly compared to the fair scheduler as shown Figure 5.3(c). We can see that the resource efficiency and fairness may not be achieved at the same time due to the heterogeneous intermediate data distributions. 


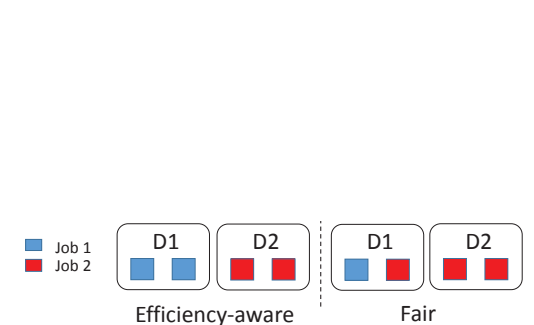

(a) Job assignment of different schedulers

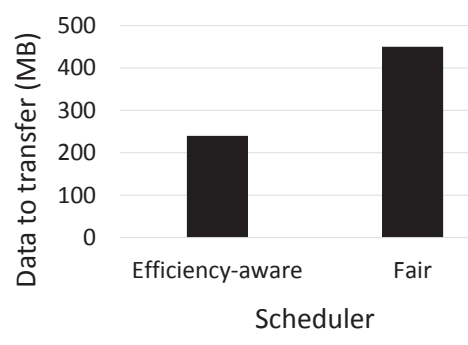

(b) Resource efficiency

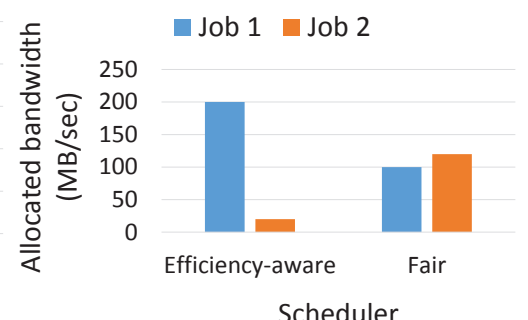

(c) Better fairness

Figure 5.4: Illustration example of the trade-offs between resource efficiency and fairness impacted by the heterogeneous WAN link bandwidth $(D 1$ and $D 2$ each has 2 available slots. Efficiency-aware scheduler assigns 2 tasks of job 1 to $D 1$ and 2 tasks of job 2 to $D 2$. Fair scheduler assigns 1 task of job 1 to $D 1,1$ task of job 2 to $D 1$ and 2 tasks of job 2 to $D 2$.)

\subsubsection{WAN Link Bandwidths for trade-off (resource efficiency vs. fairness)}

Table 5.3 shows that the bandwidths of up/down links in the three DCs are heterogeneous. The bandwidth of the down link of $D 2$ is much smaller than the bandwidths of other links.

Assume two slots in $D 1$ and two slots in $D 2$ become available. The efficiency-aware scheduler assigns two tasks of job 1 to $D 1$ and two tasks of job 2 to $D 2$ for good data locality as shown in Figure 5.4(a). As the down link of $D 2$ is the bottlenecked link, with our definition of DRF, the fair scheduler assigns 3 slot to job 2 as shown in Figure 5.4(a). Again, the efficiency-aware scheduler has less cross-DC data transfer compared to the fair scheduler while the latter guarantees fairer resource allocations, as shown in Figure 5.4(b) and 5.4(c). This example shows that heterogeneous WAN link bandwidth is another key factor which hinders multiple objectives from being optimized at the same time.

In the above examples, we only consider the resource efficiency and fairness for simplicity. In fact, more trade-off exists when considering more objectives such as job latency. For example, the shortest job first scheduler always chooses tasks from the job with the least remaining time while the efficiency-aware scheduler may choose tasks from another job with better data locality. 


\subsubsection{Summary}

Considering multiple objectives, including resource efficiency, job latency and fairness, at the same time is non-trivial for job scheduling in the geo-distributed data analytics systems. We have demonstrated that there are interesting trade-offs between them. We also observed that they are impacted by the heterogeneous intermediate data distribution and the heterogeneous WAN link bandwidths in geo-distributed data analytics systems. Due to various system operators' requirements, it is important to perform multi-objective optimization for the three discordant objectives.

\subsection{Multi-objective Optimization}

In this section, we formally define the multi-objective optimization problem for job scheduling in geodistributed data analytics systems and provide a light-weight and efficient algorithm for the problem.

We first present the metrics used to quantify the three optimization objectives, namely resource efficiency, job latency and fairness. To solve the multi-objective optimization problem, we combine the three objectives as a single objective using weighted sum and formulate the weighted-sum maximization problem as a Generalized Assignment problem (GAP).

Second, finding the optimal solution for our formulated optimization problem is too costly especially for the production workload in the geo-distributed data analytics systems. Thus, we propose an light-weight heuristic scheduling algorithm to perform the multi-objective optimization based on a weighted bipartite graph approach.

\subsubsection{Problem Formulation}

We summarize the notations used in our problem formulation in Table 5.4.

Input parameters. Consider $M$ jobs are submitted to a geo-distributed data analytics system consists $N$ DCs. The number of available nodes in DC $i$ is represented as $A_{i}$. We use $B_{i}$ to represent the bandwidth of the link $i$. The intermediate data is located in different DCs and the size of the input 


\begin{tabular}{|c|c|c|}
\hline Type & Notation & Description \\
\hline \multirow{9}{*}{ Input } & $M$ & the number of jobs \\
\hline & $N$ & the number of DCs \\
\hline & $A_{i}$ & the number of available nodes in DC $i$ \\
\hline & $B_{i}$ & the bandwidth of link $i$ \\
\hline & $I_{i j}$ & the size of input data of job $i$ in $\mathrm{DC} j$ \\
\hline & $D_{i j k}$ & $\begin{array}{l}\text { the bandwidth demand on link } k \text { when one task of job } i \text { is } \\
\text { running in DC } j\end{array}$ \\
\hline & $W_{c}$ & the weight of resource efficiency \\
\hline & $W_{q}$ & the weight of job latency \\
\hline & $W_{f}$ & the weight of fairness \\
\hline Output & $S_{i j}$ & the number of nodes job $i$ gets in DC $j$ \\
\hline \multirow{4}{*}{ Objective } & $E$ & resource efficiency \\
\hline & $Q$ & overall job latency \\
\hline & $F$ & fairness \\
\hline & $O$ & optimization goal \\
\hline \multirow{10}{*}{ Intermediate } & $L_{i j}$ & the data locality of job $i$ in DC $j$ \\
\hline & $L_{i}$ & the data locality of job $i$ \\
\hline & $R_{i j}$ & $\begin{array}{l}\text { the maximum number of running tasks in job } i \text { that can } \\
\text { be executed concurrently in one node of DC } j\end{array}$ \\
\hline & $P_{i}$ & the number of pending tasks of job $i$ \\
\hline & $c_{i}$ & CPU cores of one node in DC $i$ \\
\hline & $m_{i}$ & memory capacity of node in DC $i$ \\
\hline & $u_{i}$ & CPU demand of one task of job $i$ \\
\hline & $v_{i}$ & memory demand of one task of job $i$ \\
\hline & $H_{i j}$ & $\begin{array}{l}\text { the amount of resource gained by job } i \text { when it is running } \\
\text { in one node of DC } j\end{array}$ \\
\hline & $G_{i}$ & the amount of resource allocated to job $i$ \\
\hline
\end{tabular}

Table 5.4: Notations used in problem formulation. 
data of job $i$ in DC $j$ is denoted as $I_{i j}$. We use $D_{i j k}$ to denote the bandwidth demand on link $k$ when one task of job $i$ is running in $\mathrm{DC} j$.

Outputs. The outputs is the solution of the multi-objective optimization problem. Specifically for job scheduling, a solution is the number of nodes allocated to each submitted job from different DCs. We use $S_{i j}$ to denote the number of nodes allocated to job $i$ from DC $j$. Note that $\sum_{i} S_{i j} \leq A_{j}$.

Optimization objective. We formally define the evaluation metrics for our optimization objective.

Resource efficiency. We use data locality at DC level to represent the resource efficiency in geodistributed data analytics systems. Specifically, we calculate the data locality of job $i$ in DC $j$, denoted as $L_{i j}$ as below.

$$
L_{i j}=S_{i j} * \frac{I_{i j}}{I_{i k}}
$$

where $k=\arg \max _{j} I_{i j}$ is the DC which has the most input data of job $i$ among all DCs. We calculate the overall data locality achieved by job $i$ as $L_{i}=\sum_{j} L_{i j}$.

The resource efficiency $E$ of a scheduling decision is evaluated using the overall data locality achieved by all jobs.

$$
E=\sum_{i} L_{i}
$$

Job latency. The overall job latency objective $Q$ of a scheduling solution can be calculated as below.

$$
\begin{aligned}
Q & =\sum_{i} \sum_{j} \frac{R_{i j} * S_{i j}}{P_{i}} \\
R_{i j} & =\min \left\{\frac{c_{j}}{u_{i}}, \frac{m_{j}}{v_{i}}\right\}
\end{aligned}
$$

where $R_{i j}$ is the maximum number of running tasks of job $i$ that can concurrently run on one node of DC $j . c_{j}$ and $m_{j}$ are the number of CPU cores and memory capacity of one node in DC $j$, respectively. $u_{i}$ and $v_{i}$ represent the CPU and memory demand of one task of job $i$, respectively. 
Fairness. According to our DRF definition, we use the bandwidth of the bottlenecked link as the dominant resource for fairness quantification. That is, we calculate the amount of resource allocated to a job using the resource allocated from the bottlenecked link. Denote $H_{i j}$ as the amount of resource allocated to job $i$ from one node of DC $j$, we have $H_{i j}=B_{b t l}$, where $b t l=\arg \max _{m} \frac{D_{i j m}}{B_{m}}$ is the bottlenecked link. We quantify the fairness of a scheduling solution with the variance of allocated resource of all jobs. Denote $G_{i}$ as the amount of resource allocated to job $i$ and $F$ as the fairness objective evaluation.

$$
\begin{array}{r}
G_{i}=\sum_{j}\left\{H_{i j} * S_{i j}\right\} \\
F=\operatorname{Var}\left(G_{i}\right)
\end{array}
$$

Multi-objective optimization. We utilize the weighted sum approach to transform the multiobjective optimization problem to a single objective optimization problem, which has been widely used in the previous studies $[120,74]$. Denote the weights for resource efficiency, job latency and fairness are $W_{e}, W_{q}$ and $W_{f}$, respectively. The weights reflect system operators' preferences on different objectives. We use the normalized value in the weighted sum. Our objective is to maximize the weighted sum $O$, where

$$
O=W_{e} * E_{n}+W_{q} * Q_{n}-W_{f} * F_{n}
$$

$E_{n}, Q_{n}$ and $F_{n}$ are the normalized value of resource efficiency $E$, job latency $Q$ and fairness $F$, respectively.

\subsubsection{Light-Weight Scheduling Algorithm}

According to the above problem formulation, our optimization problem is actually a special case of GAP where the profit (allocated resource) and demand (data to transfer) of the task can vary based on the specific DC where the task is assigned. GAP has been proven to be APX-hard [117]. Thus, 


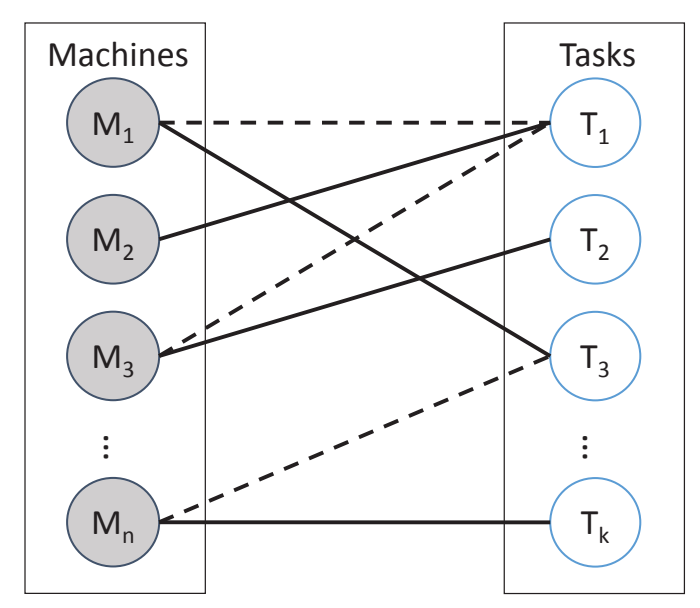

Figure 5.5: Bi-partitioned graph matching.

we propose an light-weight heuristic algorithm to provide an efficient scheduling according to the weights of different objectives.

The scheduling procedure is triggered when new resources become available. The global job scheduler decides a list of tasks to be assigned to the available resources given a set of submitted jobs. The challenge is to decide the optimal task assignment on the available resources by considering the resource efficiency, job latency and fairness at the same time.

We model this task assignment procedure as a assignment problem for a weighted bipartite graph which is shown in Figure 5.5. Each vertex in the left part of the graph represents an available machine and each vertex represents a task (or a set of tasks). In this weighted graph, each edge has an associated value which indicates the suitability of assignment of the task to the machine which is decided according to the weights of different objectives which are specified by the system operators. Finding a optimal assignment is known as a maximum weighted bipartite matching which is defined as a matching where the sum of the values of edges in the matching have a maximal value. For example, as Figure 5.5 shows, the matching edges are labeled with solid line. Each matching edge connecting a machine vertex and a task vertex means that the task is allocated to the machine.

Heuristic matching algorithm. For many practical scheduling problems involving very large 
graphs, the overhead of many existing algorithms which search for the optimum matching are too costly. Instead, we are interested in approximation algorithms for the weighted matching problem, which ideally have running time linear to the number of edges and a lower bound on the performance ratio that indicates how far the searched approximate solution is from the optimum solution. Formally, a performance ratio $c$ means the approximate solution is at least $c$ times the optimal solution in the worst case. We apply the Path Growing Algorithm [121] which is a well known and easy to implemented approximation algorithm to our job scheduling problem. The path growing algorithm has running time linear to the number of edges in the graph and has a $1 / 2$ performance ratio [121] in the worst case. Our heuristic algorithm further improves on the performance ratio while still keeping the linear running time.

Our heuristic matching algorithm consists of two steps. First, we generate a set of paths with a novel Path Growing Algorithm that is shown in Line 4-11 of Algorithm 5. The main idea is to grow a set of vertex disjoint paths in the given graph. The Path Growing Algorithm starts with a path of length 0 , i.e., a single vertex in the graph, and tries to extend the current path in one direction for as long as possible. The path is always extended along the heaviest edge currently available. Once the heaviest edge has been chosen, the current vertex is deleted in the graph to ensure that the paths are vertex disjoint. Then the vertex on the other end of the heaviest edge is chosen as the new base vertex to repeat the path growing process. If there is no possible extension to the current path from any edge, a new path is started with a new vertex.

Second, given each path generated in the first step, we use dynamic programming to get the maximum weight matching for each path. The maximum weight matching algorithm is shown in Line 12-35 of Algorithm 5. Similar to other dynamic programming algorithms, the maximum weight matching algorithm also consists of a solution find step (Line 12-24) and a solution build step (Line 25-35). Such a maximum weight matching can be computed in linear time for trees and therefore also for paths with a dynamic programming approach. We repeat the two steps until all vertices of the graph belong to some paths. 


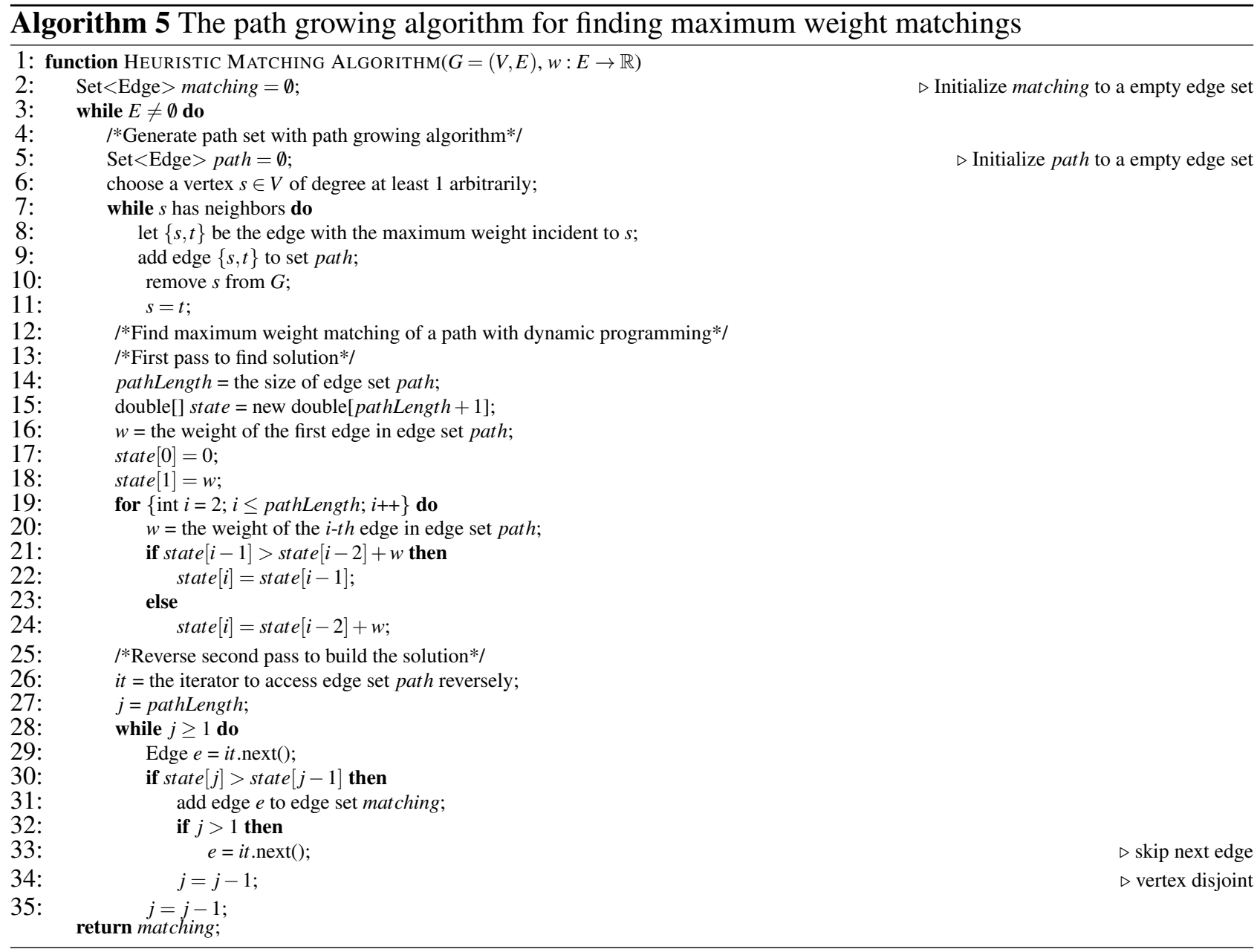


Edge weight. Assigning tasks to the nodes in different DCs have different impacts on resource efficiency, job latency and fairness. The weight of edges in the bipartite graph is decided by our optimization goal as shown in Equation 5.7. Before considering multiple objectives simultaneously, we first calculate the matching score for each individual objective. The scores for different objectives are calculated as below.

- Resource efficiency score. The input of one task can be partitioned and placed in different DCs. Executing the task on different machines can result in different amount of data transfer over the WAN network. When a task is assigned to a machine, we calculate the resource efficiency score eScore according to Equation 5.2.

- Job latency score. We calculate the job latency score $l S c o r e$ according to Equation 5.3.

- Fairness score. We apply the dominant resource fairness on the WAN links and the bottlenecked link is treated as the dominant resource. The fairness score $f S c o r e$ is calculated according to Equation 5.6.

GeoSpark attempts to simultaneously consider the above three objectives. We combine the three objectives as a single score with weighted sum of normalized eScore, lScore and $f$ Score and this score is taken as the weight of an edge in the bipartite graph.

The complexity of our algorithm is linear to the number of edges in the graph. Thus, GeoSpark is light-weight, as also demonstrated in our evaluations ( $<1 \mathrm{~ms}$ for $10 \mathrm{~K}$ tasks).

\subsubsection{Visualizing Pareto Fronts and Calibrating Weights}

To help the system operators guide the weight calibration, we need to demonstrate to them the extent of trade-offs. Therefore, we use a tool that is also used in ClavisMO [120] to periodically visualize the Pareto-optimal front of potential trade-offs by sampling workload from our system. To find a Paretooptimal front, we explore the scheduling plans with different weight combinations. Each scheduling plan is characterized by its resource efficiency, job latency and fairness. 


\subsection{Evaluation}

We evaluate GeoSpark with synthetic workload in a real testbed. We emulate the network according to the bandwidths measured from real geo-distributed data analytics systems.

\subsubsection{Experimental Setup}

Hadoop cluster. We use Apache Spark (2.11) and run the experiments in our local cluster. The local cluster consists of 10 compute nodes, each with two Intel X5675 CPUs (6 CPU cores per CPU with 3.07 GHz), 24GB DDR3 memory and 500GB 7200RPM disk drivers. These machines are connected with $10 \mathrm{~Gb} / \mathrm{sec}$ Ethernet. We mimic a geo-distributed data analytics system deployed on three Amazon EC2 regions, including US East DC, West Europe DC and Japan East DC. We use one node as the global scheduler and partition 9 nodes evenly to 3 DCs. We use the same bandwidths measured from Amazon cloud as shown in Table 5.1. We denote the US East, West Europe and Japan East DCs as DC 1, DC2 and DC3, respectively.

Workload. We synthesize a Facebook-like workload based on the distribution of jobs sizes and inter-arrival time at Facebook provided by Zaharia et. al. [24]. The workload consists of 100 jobs. The jobs can be PageRank, Terasort, Kmeans, Wordcount or Grep Search applications running on Apache Spark. We cache the input dataset in memory to accelerate the data processing with Alluxio, an open sourced, distributed, fault-tolerant and in-memory file system [122]. By default, the input size of each job is around $80 \mathrm{~GB}$. We consider a mixed workload, where each job is randomly generated from the five kinds of applications. We generate the synthetic data according to Zipf distribution with varying parameter $s$, with which we can control the skewness of data distribution. $s$ is set to 1 by default.

Compared methods. We experimentally compare GeoSpark with the following methods, including First In First Out scheduler (FIFO), Shortest Job First (SJF) scheduler, and Dominant Resource Fairness scheduler (DRF). 


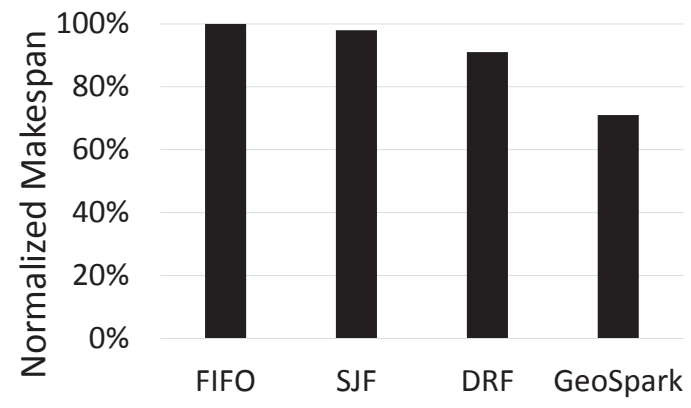

Figure 5.6: Resource efficiency comparison of different schedulers $\left(W_{e}=1, W_{q}=0, W_{f}=0\right.$ for GeoSpark).

FIFO scheduler is supported by Apache Spark. We implement SJF in Spark and extend the fair scheduler to DRF scheduler in Spark. We can configure the resource efficiency weight $W_{e}$, job latency weight $W_{q}$ and fairness weight $W_{f}$ for GeoSpark according to the importance of different optimization objectives. For example, if $W_{e}$ is set to 1 , GeoSpark actually becomes the efficiency-aware scheduler which tries to maximize the resource efficiency of the cluster at the cost of increases of average job latency and fairness loss.

\subsubsection{Overall Results}

Resource efficiency optimization. We set the resource efficiency weight $W_{e}$ to 1 to show the effectiveness of GeoSpark on optimizing resource efficiency. We compare the makespan of FIFO, SJF, DRF and GeoSpark. Figure 5.6 shows that GeoSpark achieves up to 30\% makespan reduction compared with the other schedulers. The performance improvement is achieved due to the following two reasons. First, GeoSpark achieves better data locality by considering the data distribution across DCs. Second, as shown in Figure 5.7, GeoSpark reduces the amount of data to transfer across different DCs and thus decreases the loads on WAN links which are usually the performance bottleneck in the geo-distributed data analytics systems. As Figure 5.8 shows, the average CPU utilization is only 50\% for DRF scheduler as the geo-distributed data analytics system is bottlenecked by the WAN links. GeoSpark increases the CPU utilization by performing data locality-aware optimizations. 


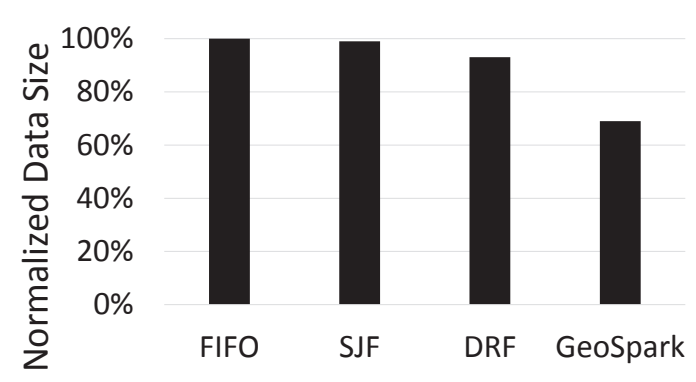

Figure 5.7: The amount of data to transfer across different DCs for different schedulers.

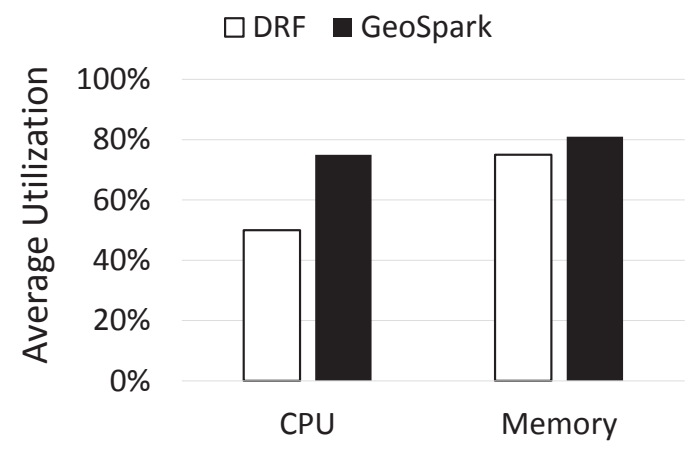

Figure 5.8: Average resource utilizations on CPU and memory for different schedulers.

Job latency optimization. We set the job latency weight $W_{q}$ to 1 to validate the job latency optimization effectiveness of GeoSpark. The optimized job completion time results are shown in Figure 5.9.

SJF scheduler obtains lower job latency compared to FIFO and DRF as short jobs are scheduled first to finish as soon as possible. GeoSpark is in fact a kind of SJF scheduler when $W_{q}$ is set to 1 . It further reduces the job latency compared to SJF by considering data locality. For example, when one node becomes available, if two jobs have the same amount of pending tasks, the job with better data locality is chosen.

Fairness optimization. We set the fairness weight $W_{f}$ to 1 to study the fairness guarantee of GeoSpark. As DRF does not consider the scarce WAN link bandwidth in resource allocation, it cannot guarantee fairness for the geo-distributed data analytics systems. To study the fairness guarantee of different schedulers, we use two jobs with the same amount of data input and skewness factor. Job 


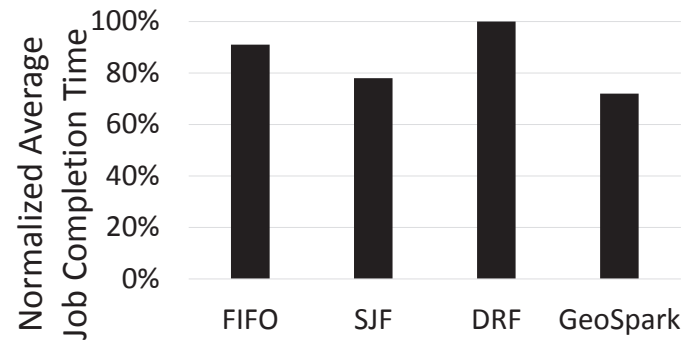

Figure 5.9: Job latency comparison of different schedulers $\left(W_{e}=0, W_{q}=1, W_{f}=0\right.$ for GeoSpark).

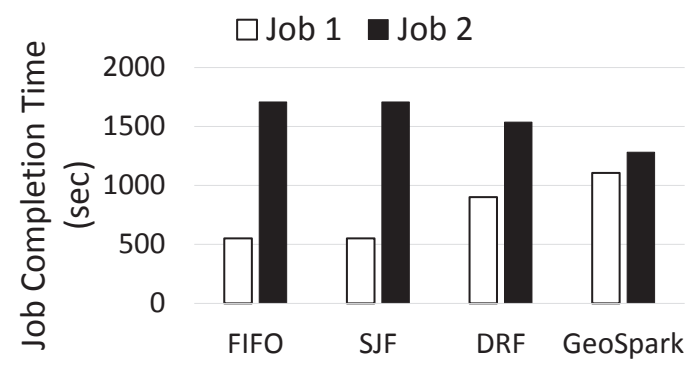

Figure 5.10: Completion time of two jobs under different schedulers $\left(W_{e}=0, W_{q}=0, W_{f}=1\right.$ for GeoSpark).

1 is skewed in DC1 and job 2 is skewed in DC 3. Figure 5.10 shows the completion time of the two jobs under different schedulers.

GeoSpark achieves the best fairness guarantee compared to other schedulers by considering the heterogeneous WAN links across different DCs. Job 1 finishes earlier because it is submitted first and the resource cannot be reallocated until some running tasks of job 1 finish. We take GeoSpark as the baseline and shows the fairness loss of the other schedulers as shown in Figure 5.11. The fairness loss is calculated as the average reduction of job completion time [13]. This demonstrates that GeoSpark provides better isolation guarantee compared to other schedulers in geo-distributed data analysis systems.

\subsubsection{Sensitivity Study}

In this subsection, we perform sensitivity studies on GeoSpark by varying the weights between different objectives, the skewness factor, network heterogeneity and the number of DCs. 


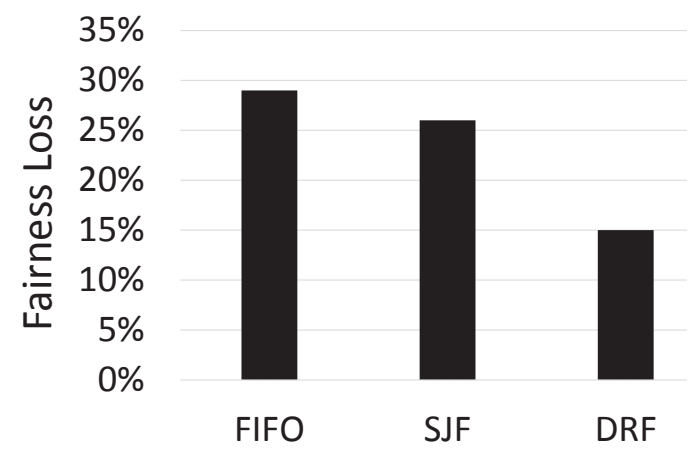

Figure 5.11: Fairness loss of different schedulers (baseline: GeoSpark $\left(W_{e}=0, W_{q}=0, W_{f}=1\right)$ )

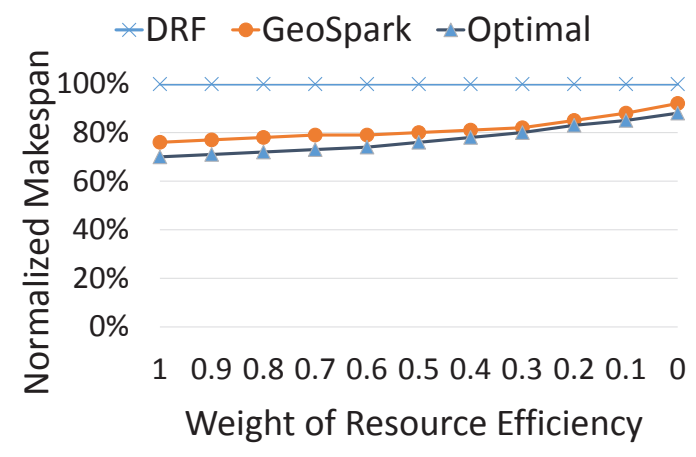

Figure 5.12: Makespan of GeoSpark by varying the weight on resource efficiency.

Makespan for different resource efficiency weights. We show the makespan of GeoSpark by varying the weight of resource efficiency. When $W_{e}$ is set to $x$, the other two weights are both set to $\frac{1-W_{e}}{2}$. In this setting, we consider fairness and job latency with the same weights.

Figure 5.12 shows that the makespan of job scheduling under GeoSpark decreases with the increase of resource efficiency weight. We also show the result of optimal scheduling solution obtained by the Kuhn-Munkras algorithm [123] (denoted as Optimal in the figure). All results are normalized to that of DRF. The makespan difference between GeoSpark and Optimal is less than 2\% under all weight settings, which validates the effectiveness of our heuristic matching algorithm.

Trade-off between resource efficiency and fairness. We show the trade-off between resource efficiency and fairness by varying the ratio between $W_{e}$ and $W_{f}$ from $(1,0)$ to $(0,1)$. Figure 5.13 shows the fairness loss and efficiency improvement results of GeoSpark under different ratios when 


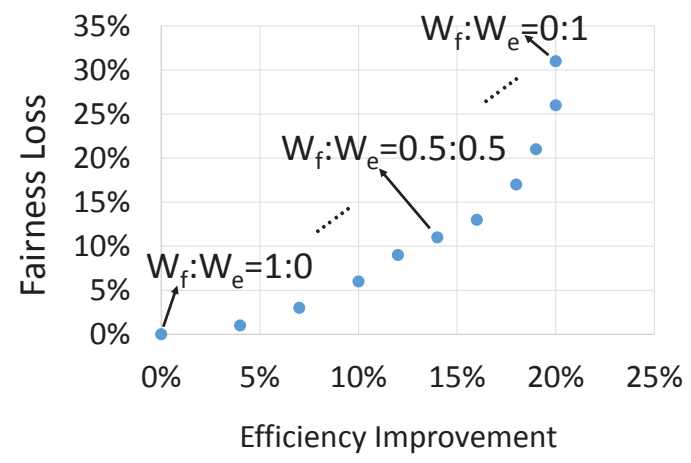

Figure 5.13: Trade-off between resource efficiency and fairness.

compared to the fair scheduler. When the improvement of resource efficiency increases, the fairness loss also increases. This demonstrates the trade-off between discordant objectives and they have non-linear relationship. It is necessary to consider the weights of multiple optimization objectives in geo-distributed data analysis systems.

Impact of data skewness. We vary the skewness of input data distribution for different skew factor $s(0.5,1,1.5)$ in the Zipf distribution function. The larger $s$ is, the more skewed the data are distributed. Figure 5.14 shows the optimization results of resource efficiency (normalized to FIFO), job latency (normalized to DRF) and fairness (compared to GeoSpark) with different schedulers. With the increase of data skewness, GeoSpark acheves larger makespan reduction and job latency reduction due to the larger optimization space for data locality.

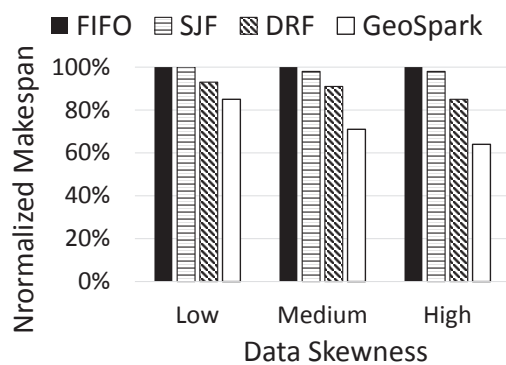

(a) Resource efficiency

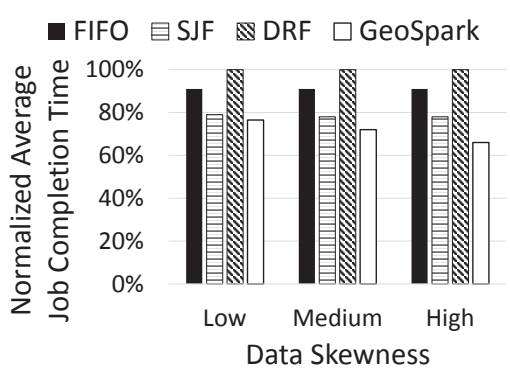

(b) Job latency

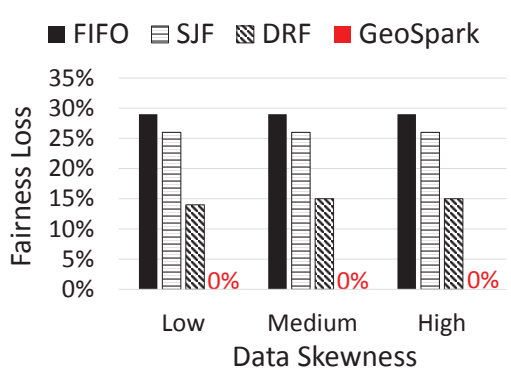

(c) Fairness

Figure 5.14: Impact of data skewness on different optimization objectives. 


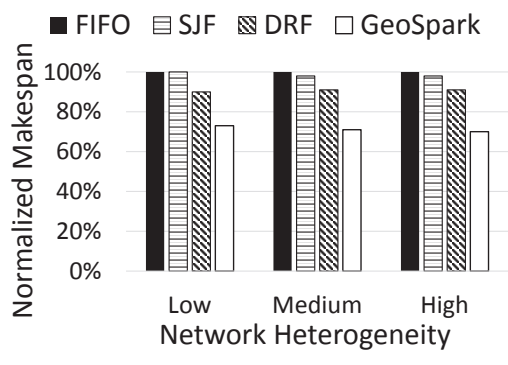

(a) Resource efficiency

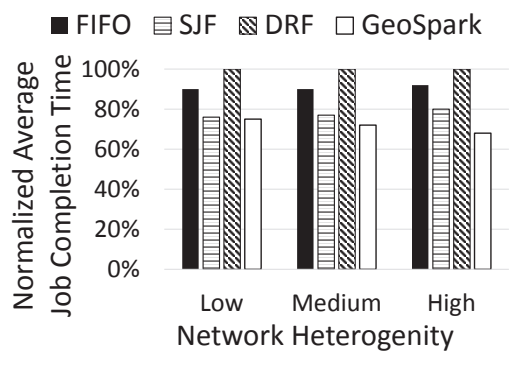

(b) Job latency

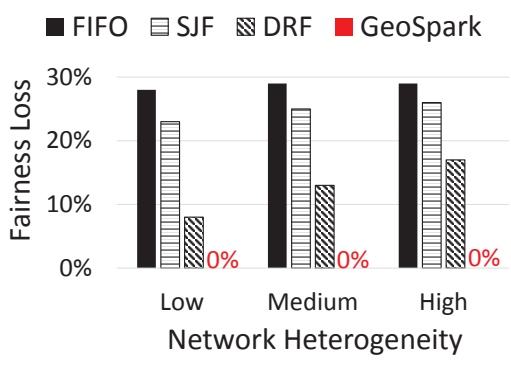

(c) Fairness

Figure 5.15: Impact of network heterogeneity on different optimization objectives.

Impact of network heterogeneity. To study the impact of network heterogeneity on different optimization objectives, we construct three distributed environments with "Low", "Medium" and "High" network heterogeneity. Specifically, in Low, the uplink and downlink of all DCs have the same bandwidths. In Medium, all DCs have the same upload/download bandwidths as those measured from the US East region of Azure. In High, we randomly select half of nodes in Medium and proportionally limit their upload/download bandwidths to a half of their original bandwidths.

Figure 5.15 shows the optimization results of resource efficiency, job latency and fairness with different schedulers. The resource efficiency and job latency objectives are less impacted by the network heterogeneity level than fairness. This is mainly because the first two objectives are also affected by workload distributions while the fairness objective is mainly affected by the dominant resource (i.e., the bottlenecked link). With the increase of network heterogeneity, GeoSpark greatly outperforms the compared schedulers on fairness guarantee.

Impact of the number of DCs. We vary the number of DCs to study its impact on different optimization objectives. The result is shown in Figure 5.16. Compared to the previous studies, the number of DCs mainly impacts the fairness objective slightly because of the link heterogeneity of different DCs. This demonstrates that GeoSpark can perform well in large-scale geo-distributed environment. 


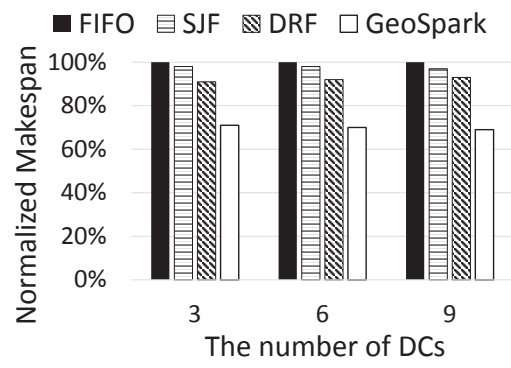

(a) Resource efficiency

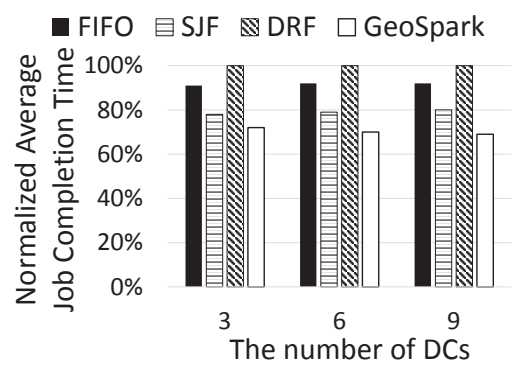

(b) Job latency

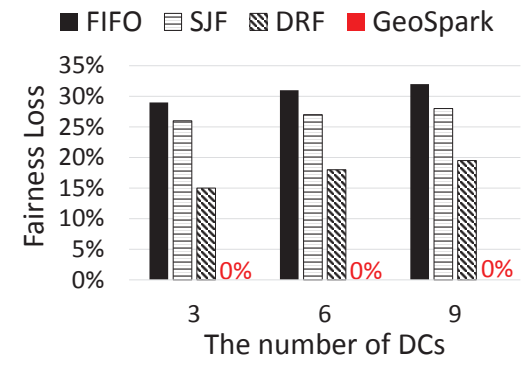

(c) Fairness

Figure 5.16: Impact of the number of DCs on different optimization objectives.

\subsubsection{Overhead Analysis}

In order to evaluate the overhead of our scheduling algorithm, we run experiments with different numbers of jobs and tasks. We present the overhead of different schedulers when there are in total $10 \mathrm{~K}$ and $50 \mathrm{~K}$ tasks from all jobs. We measure two types of scheduling overhead, namely the offline scheduling overhead for task placement at the time of job submission and the online scheduling overhead when new resources become available after some tasks are finished. The overhead results of different schedulers are shown in Table 5.5. We can see that GeoSpark has almost the same scheduling overhead compared to the existing Spark schedulers. This shows that our light-weight scheduling algorithm is able to efficient for multi-objective optimization problems.

\begin{tabular}{|l|l|l|l|}
\hline & FIFO & Fair & GeoSpark \\
\hline Offline overhead & $0.08(0.23)$ & $0.09(0.28)$ & $0.098(0.24)$ \\
\hline Online overhead & $0.03(0.03)$ & $0.03(0.03)$ & $0.03(0.04)$ \\
\hline
\end{tabular}

Table 5.5: Average scheduling overhead (ms) of different schedulers. Results are obtained when using 10K (50K) tasks.

\subsubsection{Summary}

We have the following findings from the evaluation results. First, our proposed approximate heuristic achieves significant improvements for different objectives in geo-distributed analytics systems compared to existing schedulers with very low overhead. Second, according to system operators' 
requirements, they can consider the weights of different objectives to achieve good trade-off. Third, the optimization results in geo-distributed data analytics are impacted by the weights of objectives, workload distributions and network heterogeneity. In the future work, we plan to derive (automated) approach to specify the weights for the system operators.

\subsection{Conclusion}

In geographically distributed data centers, data analytics systems have recently been developed and optimized for such geo-distributed environments. The unique features of geo-distributed environments have posed severe challenges for multi-objective optimizations for data analytics systems. In this paper, we develop an efficient framework called GeoSpark for multi-objective optimizations on geo-distributed data analytics systems. GeoSpark achieves up to 30\% makespan reduction, 28\% job latency reduction and better fairness guarantee compared with existing schedulers in Apache Spark for geo-distributed data analytics. 


\section{Chapter 6}

\section{Conclusion and Future Work}

\subsection{Conclusion}

This thesis describes our research work which mainly aims at the resource efficiency, job latency, fairness and energy consumption of the job schedulers and resource coordinators on large-scale data analytics system due to various system operators' requirements on data analytics. We perform a lot of studies on these objectives and analyze their relationships in detail. We observe that these objectives can be translated to discordant actions and thus make the multi-objective optimization quite challenging. Our preliminary studies show the interplay between multiple objectives and perform workloadaware, cost-effective and green-aware optimizations on top of Hadoop YARN and eventually develop a general and efficient framework that supports multi-objective optimizations on geo-distributed data analytics system. The experimental results demonstrate the effectiveness of our proposed systems on both real experiments and simulations.

\subsection{Future Work}

Currently, the optimizations of job schedulers and resource coordinators on our data-processing platforms are actually general for most of the data analytics systems. We have tried to utilize our optimized data analytics systems to accelerate the analysis of some elderly dataset like elderly falls 
dataset [124]. The improvement is not obvious because the elderly dataset we collected so far is rather small. We plan to collect more elderly dataset and evaluate our data analytics system with larger dataset in the future. We are planning to extend our work in three main directions in the future. First, we plan to apply big data processing technology to biomedical and health-care fields to address various issues risen from global aging. Second, as more and more applications run in containers in the large-scale cluster, efficient management of the containers incurs new challenges for the modern scheduler. Third, we play to investigate how to effectively utilize the heterogeneous hardware for the big data processing systems.

\subsubsection{Big Data Processing on Biomedical and Health-care Fields}

A large aging population imposes significant pressure to the medical and healthcare systems. A huge volume of medical and biological data have been generated and collected in the large-scale cluster. We will investigate how to apply big data processing technologies on these areas to discover new knowledge from the data and create novel approaches to improve the quality of health care especially for the elderly. For example, the new generation of efficient data processing ability and emerging hardware enable the processing of billions of DNA sequence data per day, and queries on the documented electronic health records from billions of patients.

In this study we plan to apply our data analytics system to four kinds of medical and health-care applications including bioinformatics application, clinical informatics application, imaging informatics application and public health information. First, we will study these four applications and explore the special features of these medical applications on top of the big data processing frameworks. Then, we will implement them on top of Apache spark which is the newest and most popular large-scale in-memory data processing framework. Eventually, we will utilize some medical dataset in LILY to evaluate the performance of these applications on top of Spark in our data analytics system. 


\subsubsection{Orchestration Scheduler for Containers}

Containers are rising as the new generation virtualization technology because contains share the host system's kernel with others containers which makes them easy to move and many more contains can be fitted on a host compared to original VMs. Actually, everything at Google runs in containers. Thus, efficient management of millions of containers or even more is nontrivial and challenging. Many container management systems like Docker Swarm, Mesos and kubernetes are proposed. Next, we plan to perform detailed on these system to compare them in term of performance, scalability and usability. We also will try to apply the current optimization techniques used by the existing schedulers of large-scale cluster to the orchestration scheduler for containers.

\subsubsection{Heterogeneous Data Processing with Actor Model}

Actor-based model is a simple and high-level abstractions for parallel and concurrent programming. It is an asynchronous, non-blocking and efficient event-driven programming model. The actor is the very lightweight event-driven processes in the actor-based model which naturally fits the concurrency and parallelism (several million actors per GB of heap memory). These actors can be distributed, deployed and scheduled into different computing devices, different machines and even different times slices of the physical devices without any impact on the correctness of the results. The actors are completely decoupled in terms of the space (distributed) and time (asynchronous).

Recently, modern many core hardware such as Intel Xeon Phi, graphics processing unit (GPUs) or many other coprocessors are increasingly available for general-purpose computation. These heterogeneous designs are already proved to be very efficient for many applications, such as Deep Learning and Database. Due to actors is designed from scratch especially for the highly distributed and parallel environment, they tend to be a better fit for parallel processing units. We plan to develop a new heterogeneous computing platforms based on Akka, which is the an actor-model implementation in the 
JVM platform. The actor model can simplify the development on these heterogeneous architectures and can provide efficient computing ability for the data-intensive application. 


\section{Bibliography}

[1] Y. Dong, Y. Wen, H. Hu, C. Miao, and C. Leung, "Design tradeoffs for cloud-based ambient assisted living systems," 1st International Conference on Crowd Science and Engineering, p. 7, 2016.

[2] “Google cluster data." https:// code.google.com/p/googleclusterdata.

[3] G. McNicoll, "World population ageing 1950-2050," Population and Development Review, vol. 28 , no. 4, p. 3, 2002.

[4] X. Cheng, Z. Niu, and B. He, "Silverwisdom: Towards a knowledge base for elderly people," International Journal of Information Technology, vol. 19, no. 2, 2013.

[5] W. Y. Mei and T. Zhiwei, “The elderly in singapore," Statistics Singapore Newsletter, p. 9, 2011.

[6] P. Rashidi and A. Mihailidis, "A survey on ambient-assisted living tools for older adults," IEEE Journal of Biomedical and Health Informatics, vol. 17, pp. 579-590, May 2013.

[7] J. Dean and S. Ghemawat, "Mapreduce: Simplified data processing on large clusters," Commun. ACM, vol. 51, pp. 107-113, Jan. 2008.

[8] B. Hindman, A. Konwinski, M. Zaharia, A. Ghodsi, A. D. Joseph, R. Katz, S. Shenker, and I. Stoica, "Mesos: A platform for fine-grained resource sharing in the data center," in Proceedings of the 8th USENIX Conference on Networked Systems Design and Implementation, NSDI'11, (Berkeley, CA, USA), pp. 295-308, USENIX Association, 2011.

[9] V. K. Vavilapalli, A. C. Murthy, C. Douglas, S. Agarwal, M. Konar, R. Evans, T. Graves, J. Lowe, H. Shah, S. Seth, B. Saha, C. Curino, O. O’Malley, S. Radia, B. Reed, and E. Baldeschwieler, "Apache hadoop yarn: Yet another resource negotiator," in Proceedings of the 4th 
Annual Symposium on Cloud Computing, SOCC '13, (New York, NY, USA), pp. 5:1-5:16, ACM, 2013.

[10] M. Zaharia, M. Chowdhury, T. Das, A. Dave, J. Ma, M. McCauley, M. J. Franklin, S. Shenker, and I. Stoica, "Resilient distributed datasets: A fault-tolerant abstraction for in-memory cluster computing," in Proceedings of the 9th USENIX conference on Networked Systems Design and Implementation, p. 12, USENIX Association, 2012.

[11] A. Verma, L. Pedrosa, M. Korupolu, D. Oppenheimer, E. Tune, and J. Wilkes, "Large-scale cluster management at google with borg," in Proceedings of the Tenth European Conference on Computer Systems, EuroSys '15, (New York, NY, USA), pp. 18:1-18:17, ACM, 2015.

[12] K. Elmeleegy, "Piranha: Optimizing short jobs in hadoop," Proc. VLDB Endow., vol. 6, pp. 985-996, Aug. 2013.

[13] A. Ghodsi, M. Zaharia, B. Hindman, A. Konwinski, S. Shenker, and I. Stoica, "Dominant resource fairness: Fair allocation of multiple resource types," in Proceedings of the 8th USENIX Conference on Networked Systems Design and Implementation, NSDI'11, (Berkeley, CA, USA), pp. 323-336, USENIX Association, 2011.

[14] "Industry outlook: Data center energy efficiency." http://www. datacenter journal. com/industry-outlook-data-center-energy-efficiency.

[15] R. Bianchini, "Leveraging renewable energy in data centers: Present and future," in Proceedings of the 21st International Symposium on High-Performance Parallel and Distributed Computing, HPDC '12, (New York, NY, USA), pp. 135-136, ACM, 2012.

[16] Z. Niu, S. Tang, and B. He, "Gemini: An adaptive performance-fairness scheduler for dataintensive cluster computing," in 2015 IEEE 7th International Conference on Cloud Computing Technology and Science (CloudCom), pp. 66-73, Nov 2015.

[17] Z. Niu, B. He, and F. Liu, "Not all joules are equal: Towards energy-efficient and green-aware data processing frameworks," in 2016 IEEE International Conference on Cloud Engineering (IC2E), pp. 2-11, April 2016.

[18] Z. Niu, S. Tang, and B. He, "An adaptive efficiency-fairness meta-scheduler for data-intensive computing," IEEE Transactions on Services Computing, vol. PP, no. 99, pp. 1-1, 2017. 
[19] Z. Niu and B. He, "A study of big data computing platforms: Fairness and energy consumption," in 2016 IEEE International Conference on Cloud Engineering Workshop (IC2EW), pp. 207-209, April 2016.

[20] J. Tan, A. Chin, Z. Z. Hu, Y. Hu, S. Meng, X. Meng, and L. Zhang, "Dynmr: Dynamic mapreduce with reducetask interleaving and maptask backfilling," in Proceedings of the Ninth European Conference on Computer Systems, EuroSys '14, (New York, NY, USA), pp. 2:1-2:14, ACM, 2014.

[21] J. Polo, C. Castillo, D. Carrera, Y. Becerra, I. Whalley, M. Steinder, J. Torres, and E. Ayguadé, Resource-Aware Adaptive Scheduling for MapReduce Clusters, pp. 187-207. Berlin, Heidelberg: Springer Berlin Heidelberg, 2011.

[22] X. Bu, J. Rao, and C.-z. Xu, "Interference and locality-aware task scheduling for mapreduce applications in virtual clusters," in Proceedings of the 22Nd International Symposium on Highperformance Parallel and Distributed Computing, HPDC '13, (New York, NY, USA), pp. 227238, ACM, 2013.

[23] C. Delimitrou and C. Kozyrakis, "Quasar: Resource-efficient and qos-aware cluster management," in Proceedings of the 19th International Conference on Architectural Support for Programming Languages and Operating Systems, ASPLOS '14, (New York, NY, USA), pp. 127144, ACM, 2014.

[24] M. Zaharia, D. Borthakur, J. Sen Sarma, K. Elmeleegy, S. Shenker, and I. Stoica, "Delay scheduling: A simple technique for achieving locality and fairness in cluster scheduling," in Proceedings of the 5th European Conference on Computer Systems, EuroSys '10, (New York, NY, USA), pp. 265-278, ACM, 2010.

[25] M. Y. Eltabakh, Y. Tian, F. Özcan, R. Gemulla, A. Krettek, and J. McPherson, "Cohadoop: Flexible data placement and its exploitation in hadoop," Proc. VLDB Endow., vol. 4, pp. 575585, June 2011.

[26] A. Rasmussen, V. T. Lam, M. Conley, G. Porter, R. Kapoor, and A. Vahdat, "Themis: An i/o-efficient mapreduce," in Proceedings of the Third ACM Symposium on Cloud Computing, SoCC '12, (New York, NY, USA), pp. 13:1-13:14, ACM, 2012. 
[27] S. Ibrahim, H. Jin, L. Lu, B. He, and S. Wu, "Adaptive disk i/o scheduling for mapreduce in virtualized environment," in 2011 International Conference on Parallel Processing, pp. 335344, Sept 2011.

[28] A. Shieh, S. Kandula, A. Greenberg, C. Kim, and B. Saha, "Sharing the data center network," in Proceedings of the 8th USENIX Conference on Networked Systems Design and Implementation, NSDI'11, (Berkeley, CA, USA), pp. 309-322, USENIX Association, 2011.

[29] M. Chowdhury, M. Zaharia, J. Ma, M. I. Jordan, and I. Stoica, "Managing data transfers in computer clusters with orchestra," in Proceedings of the ACM SIGCOMM 2011 Conference, SIGCOMM '11, (New York, NY, USA), pp. 98-109, ACM, 2011.

[30] "Hadoop mapreduce 1.0 - fair scheduler." http: / / hadoop. apache. org/docs/r1.2 . 1/fair_scheduler.html.

[31] M. Isard, V. Prabhakaran, J. Currey, U. Wieder, K. Talwar, and A. Goldberg, "Quincy: Fair scheduling for distributed computing clusters," in Proceedings of the ACM SIGOPS 22Nd Symposium on Operating Systems Principles, SOSP '09, (New York, NY, USA), pp. 261-276, ACM, 2009.

[32] A. Ghodsi, M. Zaharia, S. Shenker, and I. Stoica, "Choosy: Max-min fair sharing for datacenter jobs with constraints," in Proceedings of the 8th ACM European Conference on Computer Systems, EuroSys '13, (New York, NY, USA), pp. 365-378, ACM, 2013.

[33] S. Tang, B.-s. Lee, B. He, and H. Liu, "Long-term resource fairness: Towards economic fairness on pay-as-you-use computing systems," in Proceedings of the 28th ACM International Conference on Supercomputing, ICS '14, (New York, NY, USA), pp. 251-260, ACM, 2014.

[34] W. Wang, B. Li, and B. Liang, "Dominant resource fairness in cloud computing systems with heterogeneous servers," in IEEE INFOCOM 2014 - IEEE Conference on Computer Communications, pp. 583-591, April 2014.

[35] H. Liu and B. He, "Reciprocal resource fairness: Towards cooperative multiple-resource fair sharing in iaas clouds," in Proceedings of the International Conference for High Performance Computing, Networking, Storage and Analysis, SC '14, (Piscataway, NJ, USA), pp. 970-981, IEEE Press, 2014. 
[36] H. Liu and B. He, "F2c: Enabling fair and fine-grained resource sharing in multi-tenant iaas clouds," IEEE Transactions on Parallel and Distributed Systems, vol. 27, pp. 2589-2602, Sept 2016.

[37] M. Zaharia, M. Chowdhury, M. J. Franklin, S. Shenker, and I. Stoica, "Spark: Cluster computing with working sets," in Proceedings of the 2Nd USENIX Conference on Hot Topics in Cloud Computing, HotCloud'10, (Berkeley, CA, USA), pp. 10-10, USENIX Association, 2010.

[38] Q. Pu, G. Ananthanarayanan, P. Bodik, S. Kandula, A. Akella, P. Bahl, and I. Stoica, "Low latency geo-distributed data analytics," in Proceedings of the 2015 ACM Conference on Special Interest Group on Data Communication, SIGCOMM '15, (New York, NY, USA), pp. 421-434, ACM, 2015.

[39] C.-C. Hung, L. Golubchik, and M. Yu, "Scheduling jobs across geo-distributed datacenters," in Proceedings of the Sixth ACM Symposium on Cloud Computing, SoCC '15, (New York, NY, USA), pp. 111-124, ACM, 2015.

[40] J. Leverich and C. Kozyrakis, "On the energy (in)efficiency of hadoop clusters," SIGOPS Oper. Syst. Rev., vol. 44, pp. 61-65, Mar. 2010.

[41] R. T. Kaushik and M. Bhandarkar, "Greenhdfs: towards an energy-conserving, storageefficient, hybrid hadoop compute cluster,' in In Proceedings of the 2010 international conference on Power aware computing and systems, pp. 1-9, 2010.

[42] B. Shi and A. Srivastava, "Thermal and power-aware task scheduling for hadoop based storage centric datacenters," in Proceedings of the International Conference on Green Computing, GREENCOMP '10, (Washington, DC, USA), pp. 73-83, IEEE Computer Society, 2010.

[43] J. Kim and D. Rotem, "Energy proportionality for disk storage using replication," in Proceedings of the 14th International Conference on Extending Database Technology, EDBT/ICDT '11, (New York, NY, USA), pp. 81-92, ACM, 2011.

[44] J. C. Y. Chou, T. H. Lai, J. Kim, and D. Rotem, "Exploiting replication for energy-aware scheduling in disk storage systems," IEEE Transactions on Parallel and Distributed Systems, vol. 26, pp. 2734-2749, Oct 2015.

[45] W. Lang and J. M. Patel, "Energy management for mapreduce clusters," Proc. VLDB Endow., vol. 3, pp. 129-139, Sept. 2010. 
[46] Y. Chen, S. Alspaugh, D. Borthakur, and R. Katz, "Energy efficiency for large-scale mapreduce workloads with significant interactive analysis," in Proceedings of the 7th ACM European Conference on Computer Systems, EuroSys '12, (New York, NY, USA), pp. 43-56, ACM, 2012.

[47] W. Lang, J. M. Patel, and S. Shankar, "Wimpy node clusters: What about non-wimpy workloads?" in Proceedings of the Sixth International Workshop on Data Management on New Hardware, DaMoN '10, (New York, NY, USA), pp. 47-55, ACM, 2010.

[48] R. Urgaonkar, B. Urgaonkar, M. J. Neely, and A. Sivasubramaniam, "Optimal power cost management using stored energy in data centers," SIGMETRICS Perform. Eval. Rev., vol. 39, pp. 181-192, June 2011.

[49] W. Deng, F. Liu, H. Jin, and C. Wu, "Smartdpss: Cost-minimizing multi-source power supply for datacenters with arbitrary demand," in Proceedings of the 2013 IEEE 33rd International Conference on Distributed Computing Systems, ICDCS '13, (Washington, DC, USA), pp. 420 429, IEEE Computer Society, 2013.

[50] A. Qureshi, R. Weber, H. Balakrishnan, J. Guttag, and B. Maggs, "Cutting the electric bill for internet-scale systems," in Proceedings of the ACM SIGCOMM 2009 Conference on Data Communication, SIGCOMM '09, (New York, NY, USA), pp. 123-134, ACM, 2009.

[51] K. Le, R. Bianchini, M. Martonosi, and T. D. Nguyen, "Cost-and energy-aware load distribution across data centers," Proceedings of HotPower, p. 5, 2009.

[52] J. Yu and R. Buyya, "Scheduling scientific workflow applications with deadline and budget constraints using genetic algorithms," Sci. Program., vol. 14, pp. 217-230, Dec. 2006.

[53] X. Tang, C. Chen, and B. He, "Green-aware workload scheduling in geographically distributed data centers," in Proceedings of the 2012 IEEE 4th International Conference on Cloud Computing Technology and Science (CloudCom), CLOUDCOM '12, (Washington, DC, USA), pp. 8289, IEEE Computer Society, 2012.

[54] G. Ananthanarayanan, C. Douglas, R. Ramakrishnan, S. Rao, and I. Stoica, "True elasticity in multi-tenant data-intensive compute clusters," in Proceedings of the Third ACM Symposium on Cloud Computing, SoCC '12, (New York, NY, USA), pp. 24:1-24:7, ACM, 2012. 
[55] B. Cho, M. Rahman, T. Chajed, I. Gupta, C. Abad, N. Roberts, and P. Lin, "Natjam: Design and evaluation of eviction policies for supporting priorities and deadlines in mapreduce clusters," in Proceedings of the 4th Annual Symposium on Cloud Computing, SOCC '13, (New York, NY, USA), pp. 6:1-6:17, ACM, 2013.

[56] I. n. Goiri, K. Le, M. E. Haque, R. Beauchea, T. D. Nguyen, J. Guitart, J. Torres, and R. Bianchini, "Greenslot: Scheduling energy consumption in green datacenters," in Proceedings of 2011 International Conference for High Performance Computing, Networking, Storage and Analysis, SC '11, (New York, NY, USA), pp. 20:1-20:11, ACM, 2011.

[57] I. n. Goiri, K. Le, T. D. Nguyen, J. Guitart, J. Torres, and R. Bianchini, "Greenhadoop: Leveraging green energy in data-processing frameworks," in Proceedings of the 7th ACM European Conference on Computer Systems, EuroSys '12, (New York, NY, USA), pp. 57-70, ACM, 2012.

[58] B. Aksanli, J. Venkatesh, L. Zhang, and T. Rosing, "Utilizing green energy prediction to schedule mixed batch and service jobs in data centers," in Proceedings of the 4th Workshop on Power-Aware Computing and Systems, HotPower '11, (New York, NY, USA), pp. 5:1-5:5, ACM, 2011.

[59] A. Krioukov, C. Goebel, S. Alspaugh, Y. Chen, D. Culler, and Y. Katz, "Integrating renewable energy using data analytics systems: Challenges and opportunities," in In Bulletin of the IEEE Computer Society Technical Committee, 2011.

[60] N. Sharma, S. Barker, D. Irwin, and P. Shenoy, "Blink: Managing server clusters on intermittent power," SIGARCH Comput. Archit. News, vol. 39, pp. 185-198, Mar. 2011.

[61] R. Singh, D. Irwin, P. Shenoy, and K. K. Ramakrishnan, "Yank: Enabling green data centers to pull the plug," in Proceedings of the 10th USENIX Conference on Networked Systems Design and Implementation, nsdi'13, (Berkeley, CA, USA), pp. 143-156, USENIX Association, 2013.

[62] C. Li, A. Qouneh, and T. Li, "iswitch: Coordinating and optimizing renewable energy powered server clusters," in Proceedings of the 39th Annual International Symposium on Computer Architecture, ISCA '12, (Washington, DC, USA), pp. 512-523, IEEE Computer Society, 2012.

[63] I. n. Goiri, W. Katsak, K. Le, T. D. Nguyen, and R. Bianchini, "Parasol and greenswitch: Managing datacenters powered by renewable energy," SIGARCH Comput. Archit. News, vol. 41, pp. 51-64, Mar. 2013. 
[64] M. E. Haque, K. Le, . Goiri, R. Bianchini, and T. D. Nguyen, "Providing green slas in high performance computing clouds," in 2013 International Green Computing Conference Proceedings, pp. 1-11, June 2013.

[65] C. Chen, B. He, X. Tang, C. Chen, and Y. Liu, "Green databases through integration of renewable energy," in Conference on Innovative Data Systems Research, p. 10, 2013.

[66] C. Li, R. Zhou, and T. Li, "Enabling distributed generation powered sustainable highperformance data center," in Proceedings of the 2013 IEEE 19th International Symposium on High Performance Computer Architecture (HPCA), HPCA '13, (Washington, DC, USA), pp. 35-46, IEEE Computer Society, 2013.

[67] S. Govindan, A. Sivasubramaniam, and B. Urgaonkar, "Benefits and limitations of tapping into stored energy for datacenters," in Proceedings of the 38th Annual International Symposium on Computer Architecture, ISCA '11, (New York, NY, USA), pp. 341-352, ACM, 2011.

[68] V. Kontorinis, L. E. Zhang, B. Aksanli, J. Sampson, H. Homayoun, E. Pettis, D. M. Tullsen, and T. S. Rosing, "Managing distributed ups energy for effective power capping in data centers," in Proceedings of the 39th Annual International Symposium on Computer Architecture, ISCA '12, (Washington, DC, USA), pp. 488-499, IEEE Computer Society, 2012.

[69] M. E. Haque, I. Goiri, R. Bianchini, and T. D. Nguyen, "Greenpar: Scheduling parallel high performance applications in green datacenters," in Proceedings of the 29th ACM on International Conference on Supercomputing, ICS '15, (New York, NY, USA), pp. 217-227, ACM, 2015.

[70] C. Joe-Wong, S. Sen, T. Lan, and M. Chiang, "Multiresource allocation: Fairness-efficiency tradeoffs in a unifying framework," IEEE/ACM Trans. Netw., vol. 21, pp. 1785-1798, Dec. 2013.

[71] W. Wang, C. Feng, B. Li, and B. Liang, "On the fairness-efficiency tradeoff for packet processing with multiple resources," in Proceedings of the 10th ACM International on Conference on Emerging Networking Experiments and Technologies, CoNEXT '14, (New York, NY, USA), pp. 235-248, ACM, 2014. 
[72] W. Wang, C. Feng, B. Li, and B. Liang, "On the fairness-efficiency tradeoff for packet processing with multiple resources," in Proceedings of the 10th ACM International on Conference on Emerging Networking Experiments and Technologies, CoNEXT '14, (New York, NY, USA), pp. 235-248, ACM, 2014.

[73] R. Grandl, G. Ananthanarayanan, S. Kandula, S. Rao, and A. Akella, "Multi-resource packing for cluster schedulers," in Proceedings of the 2014 ACM Conference on SIGCOMM, SIGCOMM '14, (New York, NY, USA), pp. 455-466, ACM, 2014.

[74] R. Grandl, M. Chowdhury, A. Akella, and G. Ananthanarayanan, "Altruistic scheduling in multi-resource clusters," in Proceedings of the 12th USENIX Conference on Operating Systems Design and Implementation, OSDI' 16, (Berkeley, CA, USA), pp. 65-80, USENIX Association, 2016.

[75] R. Grandl, S. Kandula, S. Rao, A. Akella, and J. Kulkarni, "Graphene: Packing and dependency-aware scheduling for data-parallel clusters," in Proceedings of the 12th USENIX Conference on Operating Systems Design and Implementation, OSDI'16, (Berkeley, CA, USA), pp. 81-97, USENIX Association, 2016.

[76] A. Rényi et al., "On measures of entropy and information," in Proceedings of the fourth Berkeley symposium on mathematical statistics and probability, vol. 1, p. 15, 1961.

[77] H. Arabnejad and J. Barbosa, "Fairness resource sharing for dynamic workflow scheduling on heterogeneous systems," in 2012 IEEE 10th International Symposium on Parallel and Distributed Processing with Applications, pp. 633-639, July 2012.

[78] H. Zhao and R. Sakellariou, "Scheduling multiple dags onto heterogeneous systems," in Proceedings of the 20th International Conference on Parallel and Distributed Processing, IPDPS'06, (Washington, DC, USA), pp. 159-159, IEEE Computer Society, 2006.

[79] K. Chard and K. Bubendorfer, "A distributed economic meta-scheduler for the grid," in 2008 Eighth IEEE International Symposium on Cluster Computing and the Grid (CCGRID), pp. 542-547, May 2008.

[80] A. Foltzer, A. Kulkarni, R. Swords, S. Sasidharan, E. Jiang, and R. Newton, "A meta-scheduler for the par-monad: Composable scheduling for the heterogeneous cloud," in Proceedings of the 17th ACM SIGPLAN International Conference on Functional Programming, ICFP '12, (New York, NY, USA), pp. 235-246, ACM, 2012. 
[81] M. Banikazemi, D. Poff, and B. Abali, "Pam: A novel performance/power aware metascheduler for multi-core systems," in Proceedings of the 2008 ACM/IEEE Conference on Supercomputing, SC '08, (Piscataway, NJ, USA), pp. 39:1-39:12, IEEE Press, 2008.

[82] S. R. Safavian and D. Landgrebe, "A survey of decision tree classifier methodology," IEEE Transactions on Systems, Man, and Cybernetics, vol. 21, pp. 660-674, May 1991.

[83] L. E. Raileanu and K. Stoffel, "Theoretical comparison between the gini index and information gain criteria," Annals of Mathematics and Artificial Intelligence, vol. 41, pp. 77-93, May 2004.

[84] J. R. Quinlan, “Induction of decision trees,” Mach. Learn., vol. 1, pp. 81-106, Mar. 1986.

[85] "Facebook swim trace." https://github.com/SWIMP rojectUCB/SWIM.

[86] "Hive performance benchmarks." https://issues.apache.org/jira/browse/ HIVE-396.

[87] T. Fawcett, "An introduction to roc analysis," Pattern Recogn. Lett., vol. 27, pp. 861-874, June 2006.

[88] D. G. Luenberger, Introduction to linear and nonlinear programming, vol. 28. Addison-Wesley Reading, MA, 1973.

[89] I. Hayashi, T. Maeda, A. Bastian, and L. C. Jain, "Generation of fuzzy decision trees by fuzzy id3 with adjusting mechanism of and/or operators," in 1998 IEEE International Conference on Fuzzy Systems Proceedings. IEEE World Congress on Computational Intelligence (Cat. No.98CH36228), vol. 1, pp. 681-685 vol.1, May 1998.

[90] C. Stewart and K. Shen, "Some joules are more precious than others: Managing renewable energy in the datacenter," in Proceedings of the workshop on power aware computing and systems, p. 5, IEEE, 2009.

[91] D. Schneider and Q. Hardy, "Under the hood at google and facebook," IEEE Spectrum, vol. 48, pp. 63-67, June 2011.

[92] "Google throws open doors to its top-secret data center." http://www.wired.com/ 2012/10/ff-inside-google-data-center/all. 
[93] "Microsoft reveals its specialty servers racks." http:// www. datacenterknowledge.com/archives/2011/04/25/ microsoft-reveals-its-specialty-servers-racks.

[94] C. Li, W. Zhang, C.-B. Cho, and T. Li, "Solarcore: Solar energy driven multi-core architecture power management," in Proceedings of the 2011 IEEE 17th International Symposium on High Performance Computer Architecture, HPCA '11, (Washington, DC, USA), pp. 205-216, IEEE Computer Society, 2011.

[95] D. S. Palasamudram, R. K. Sitaraman, B. Urgaonkar, and R. Urgaonkar, "Using batteries to reduce the power costs of internet-scale distributed networks," in Proceedings of the Third ACM Symposium on Cloud Computing, SoCC '12, (New York, NY, USA), pp. 11:1-11:14, ACM, 2012.

[96] G. Ananthanarayanan, S. Kandula, A. Greenberg, I. Stoica, Y. Lu, B. Saha, and E. Harris, "Reining in the outliers in map-reduce clusters using mantri," in Proceedings of the 9th USENIX Conference on Operating Systems Design and Implementation, OSDI' 10, (Berkeley, CA, USA), pp. 265-278, USENIX Association, 2010.

[97] Y. Chen, A. Ganapathi, R. Griffith, and R. Katz, "The case for evaluating mapreduce performance using workload suites," in Proceedings of the 2011 IEEE 19th Annual International Symposium on Modelling, Analysis, and Simulation of Computer and Telecommunication Systems, MASCOTS '11, (Washington, DC, USA), pp. 390-399, IEEE Computer Society, 2011.

[98] S. S. Elnaffar, Towards Workload-aware Dbmss: Identifying Workload Type and Predicting Its Change. PhD thesis, Kingston, Ont., Canada, Canada, 2004. AAINQ99940.

[99] S. Di, D. Kondo, and W. Cirne, "Host load prediction in a google compute cloud with a bayesian model," in Proceedings of the International Conference on High Performance Computing, Networking, Storage and Analysis, SC '12, (Los Alamitos, CA, USA), pp. 21:1-21:11, IEEE Computer Society Press, 2012.

[100] S. Jebaraj and S. Iniyan, "A review of energy models," Renewable and Sustainable Energy Reviews, vol. 10, no. 4, pp. 281 - 311, 2006. 
[101] M. Bartlett, A. M. Frisch, Y. Hamadi, I. Miguel, S. A. Tarim, and C. Unsworth, "The temporal knapsack problem and its solution," in Proceedings of the Second International Conference on Integration of AI and OR Techniques in Constraint Programming for Combinatorial Optimization Problems, CPAIOR'05, (Berlin, Heidelberg), pp. 34-48, Springer-Verlag, 2005.

[102] V. Jalaparti, H. Ballani, P. Costa, T. Karagiannis, and A. Rowstron, "Bridging the tenantprovider gap in cloud services," in Proceedings of the Third ACM Symposium on Cloud Computing, SoCC '12, (New York, NY, USA), pp. 10:1-10:14, ACM, 2012.

[103] B. Palanisamy, A. Singh, L. Liu, and B. Langston, "Cura: A cost-optimized model for mapreduce in a cloud," in Proceedings of the 2013 IEEE 27th International Symposium on Parallel and Distributed Processing, IPDPS '13, (Washington, DC, USA), pp. 1275-1286, IEEE Computer Society, 2013.

[104] H. Herodotou and S. Babu, "Profiling, what-if analysis, and cost-based optimization of mapreduce programs," Proceedings of the International Conference on Very Large Data Bases, vol. 4, no. 11, p. 12, 2011.

[105] G. Ananthanarayanan and R. H. Katz, "Greening the switch," in Proceedings of the 2008 Conference on Power Aware Computing and Systems, HotPower'08, (Berkeley, CA, USA), pp. 7-7, USENIX Association, 2008.

[106] H. Ballani, P. Costa, T. Karagiannis, and A. Rowstron, "Towards predictable datacenter networks," in Proceedings of the ACM SIGCOMM 2011 Conference, SIGCOMM '11, (New York, NY, USA), pp. 242-253, ACM, 2011.

[107] M. J. Neely, A. S. Tehrani, and A. G. Dimakis, "Efficient algorithms for renewable energy allocation to delay tolerant consumers," in 2010 First IEEE International Conference on Smart Grid Communications, pp. 549-554, Oct 2010.

[108] D. Meisner, C. M. Sadler, L. A. Barroso, W.-D. Weber, and T. F. Wenisch, "Power management of online data-intensive services," in Proceedings of the 38th Annual International Symposium on Computer Architecture, ISCA '11, (New York, NY, USA), pp. 319-330, ACM, 2011.

[109] "Amazon global infrastructure." http://aws.amazon.com/about-aws/ global-infrastructure. 
[110] "Microsoft cloud platform." http://www.microsoft.com/en-us/ server-cloud/cloudos/global-datacenters.aspx.

[111] S. Jain, A. Kumar, S. Mandal, J. Ong, L. Poutievski, A. Singh, S. Venkata, J. Wanderer, J. Zhou, M. Zhu, J. Zolla, U. Hölzle, S. Stuart, and A. Vahdat, "B4: Experience with a globallydeployed software defined wan," in Proceedings of the ACM SIGCOMM 2013 Conference on SIGCOMM, SIGCOMM '13, (New York, NY, USA), pp. 3-14, ACM, 2013.

[112] P. Mohan, A. Thakurta, E. Shi, D. Song, and D. Culler, "Gupt: Privacy preserving data analysis made easy," in Proceedings of the 2012 ACM SIGMOD International Conference on Management of Data, SIGMOD '12, (New York, NY, USA), pp. 349-360, ACM, 2012.

[113] A. Vulimiri, C. Curino, P. B. Godfrey, T. Jungblut, J. Padhye, and G. Varghese, "Global analytics in the face of bandwidth and regulatory constraints," in Proceedings of the 12th USENIX Conference on Networked Systems Design and Implementation, NSDI'15, (Berkeley, CA, USA), pp. 323-336, USENIX Association, 2015.

[114] A. Vulimiri, C. Curino, P. B. Godfrey, T. Jungblut, K. Karanasos, J. Padhye, and G. Varghese, "Wanalytics: Geo-distributed analytics for a data intensive world," in Proceedings of the 2015 ACM SIGMOD International Conference on Management of Data, SIGMOD '15, (New York, NY, USA), pp. 1087-1092, ACM, 2015.

[115] J. C. Corbett, J. Dean, M. Epstein, A. Fikes, C. Frost, J. J. Furman, S. Ghemawat, A. Gubarev, C. Heiser, P. Hochschild, W. Hsieh, S. Kanthak, E. Kogan, H. Li, A. Lloyd, S. Melnik, D. Mwaura, D. Nagle, S. Quinlan, R. Rao, L. Rolig, Y. Saito, M. Szymaniak, C. Taylor, R. Wang, and D. Woodford, "Spanner: Google's globally distributed database," ACM Trans. Comput. Syst., vol. 31, pp. 8:1-8:22, Aug. 2013.

[116] A. Rabkin, M. Arye, S. Sen, V. S. Pai, and M. J. Freedman, "Aggregation and degradation in jetstream: Streaming analytics in the wide area," in Proceedings of the 11th USENIX Conference on Networked Systems Design and Implementation, NSDI'14, (Berkeley, CA, USA), pp. 275-288, USENIX Association, 2014.

[117] C. Chekuri and S. Khanna, "A ptas for the multiple knapsack problem," in Proceedings of the Eleventh Annual ACM-SIAM Symposium on Discrete Algorithms, SODA '00, (Philadelphia, PA, USA), pp. 213-222, Society for Industrial and Applied Mathematics, 2000. 
[118] "Measuring internet congestion: A preliminary report." https://ipp.mit.edu/sites / default/files/documents/Congestion-handout-final.pdf.

[119] M. Chowdhury, Y. Zhong, and I. Stoica, "Efficient coflow scheduling with varys," in Proceedings of the 2014 ACM Conference on SIGCOMM, SIGCOMM '14, (New York, NY, USA), pp. 443-454, ACM, 2014.

[120] S. Blagodurov, A. Fedorova, E. Vinnik, T. Dwyer, and F. Hermenier, "Multi-objective job placement in clusters," in Proceedings of the International Conference for High Performance Computing, Networking, Storage and Analysis, SC'15, (New York, NY, USA), pp. 66:1-66:12, ACM, 2015.

[121] D. E. Drake and S. Hougardy, "A simple approximation algorithm for the weighted matching problem," Inf. Process. Lett., vol. 85, pp. 211-213, Feb. 2003.

[122] H. Li, A. Ghodsi, M. Zaharia, S. Shenker, and I. Stoica, "Tachyon: Reliable, memory speed storage for cluster computing frameworks," in Proceedings of the ACM Symposium on Cloud Computing, SOCC '14, (New York, NY, USA), pp. 6:1-6:15, ACM, 2014.

[123] J. Munkres, "Algorithms for the assignment and transportation problems," Journal of the Society for Industrial and Applied Mathematics, vol. 5, no. 1, pp. 32-38, 1957.

[124] "Elderly falls dataset." https:// catalog.data.gov/dataset/elderly-falls. 